REPRESENTATION THEORY

An Electronic Journal of the American Mathematical Society

Volume 3, Pages 373-415 (October 13, 1999)

S $1088-4165(99) 00012-6$

\title{
THE FIVE EXCEPTIONAL SIMPLE LIE SUPERALGEBRAS OF VECTOR FIELDS AND THEIR FOURTEEN REGRADINGS
}

\author{
IRINA SHCHEPOCHKINA
}

\begin{abstract}
The five simple exceptional complex Lie superalgebras of vector fields are described. One of them, kas, is new; the other four are explicitly described for the first time. All nonisomorphic maximal subalgebras of finite codimension of these Lie superalgebras, i.e., all other realizations of these Lie superalgebras as Lie superalgebras of vector fields, are also described; there are 14 of them altogether. All of the exceptional Lie superalgebras are obtained with the help of the Cartan prolongation or a generalized prolongation.
\end{abstract}

\section{INTRODUCTION}

V. Kac conjectured [K1] (Theorem 10 and Conjecture 1) that infinite dimensional simple Lie superalgebras of vector fields with polynomial or formal coefficients are only straightforward analogs of the four well-known Cartan series $\mathfrak{v e c t}(n), \mathfrak{s} \mathfrak{v e c t}(n)$, $\mathfrak{h}(2 n)$ and $\mathfrak{k}(2 n+1)$ (of all, divergence-free, hamiltonian and contact vector fields, respectively, realized on the space of dimension indicated). Since superdimension is a pair of numbers, Kac's examples of simple vectorial Lie superalgebras "double" Cartan's list of simple vectorial Lie algebras.

It soon became clear ([L1], [ALSh]) that the actual list of simple vectorial Lie superalgebras "doubles" that of Cartan twice, not once (the nondirect "super" counterparts $\mathfrak{m}$ and $\mathfrak{s m}$ of $\mathfrak{k}$ as well as $\mathfrak{k}$ and $\mathfrak{s l e}$ - the counterparts of $\mathfrak{h}$ - were discovered).

Moreover, even the Lie superalgebras of the four well-known series (vect, $\mathfrak{s v e c t}$, $\mathfrak{h}$ and $\mathfrak{k}$ ) have, in addition to the dimension of the superspace on which they are usually realized, one more discrete parameter governing their other, nonstandard, realizations $([\mathrm{ALSh}])$. In other words, one Lie superalgebra of vector fields has several (but not too many!) different nonisomorphic realizations as a filtered Lie superalgebra.

Furthermore, several of these Lie superalgebras have deformations (see [L2], [Ko1], [Ko2], [L3], [LSh2]). Some of these deformed Lie superalgebras are very interesting due to their applications to physics.

Received by the editors December 20, 1996 and, in revised form, October 20, 1998 and August 16, 1999.

1991 Mathematics Subject Classification. Primary 17A70; Secondary 17B35.

Key words and phrases. Lie superalgebra, Cartan prolongation, spinor representation.

I am thankful to D. Leites for raising the problem and help; to INTAS grant 96-0538 and NFR for financial support; University of Twente and Stockholm University for hospitality. Computer experiments by G. Post and P. Grozman encouraged me to carry on with unbearable calculations.

(C)1999 American Mathematical Society 
Next, three exceptional vectorial algebras were discovered [Sh1], followed by a fourth exception [Sh2]. The purpose of this note is to give a more lucid description of these exceptions, and introduce the most remarkable fifth exception (kas). (For a related construction of Lie superalgebras of string theories cf. [GLS] and [CK].)

In this note the ground field is $\mathbb{C}$. First, we recall the background from Linear Algebra in Superspaces. Then we recall the definition of the main tool in the construction of our examples: the notion of Cartan prolongation and its generalizations (cf. [Sh1]). We also recall some facts from the classification of simple Lie superalgebras of vector fields, cf. [L2] and [L3].

The main result of this paper is the discovery and a description of the five exceptional simple Lie superalgebras of vector fields and their fourteen $\mathrm{W}$-regradings.

Here are the names of the exceptional simple Lie superalgebras, the description in terms of (generalized) Cartan prolongation (for definitions see sec. 0.5), a natural minimal simple ambient and the values $r$ of the regradings (K stands for a grading consistent with parity); these regradings are described in $\S 7$; the indeterminates, whose degrees are fixed below, are introduced, respectively: in $\S 5$ for $\mathfrak{v l e}$, in $\S 3$ for $\mathfrak{k} \mathfrak{a s}$, in Appendix 3 for $\mathfrak{m} \mathfrak{b}$ and $\mathfrak{k} \mathfrak{s} \mathfrak{e}$ :

1) $\mathfrak{v l e}(4 \mid 3 ; r)=(\Pi(\Lambda(3) / \mathbb{C} \cdot 1), \mathfrak{c v e c t}(0 \mid 3))_{*} \subset \mathfrak{v e c t}(4 \mid 3), \quad r=0,1, K$

$r=0: \operatorname{deg} y=\operatorname{deg} u_{i}=\operatorname{deg} \xi_{i}=1$

$r=1: \operatorname{deg} y=\operatorname{deg} \xi_{1}=0, \operatorname{deg} u_{2}=\operatorname{deg} u_{3}=\operatorname{deg} \xi_{2}=\operatorname{deg} \xi_{3}=1, \operatorname{deg} u_{1}=2$

$r=K: \operatorname{deg} y=0, \operatorname{deg} u_{i}=2 ; \operatorname{deg} \xi_{i}=1$

2) $\mathfrak{v a \mathfrak { s }}(4 \mid 4)=(\operatorname{spin}, \mathfrak{a} \mathfrak{s})_{*} \subset \mathfrak{v e c t}(4 \mid 4)$

3) $\mathfrak{k} \mathfrak{a s} \subset \mathfrak{k}(1 \mid 6 ; r), \quad r=0(K), 1,3 \xi, 3 \eta$

$r=0(K): \operatorname{deg} t=2, \operatorname{deg} \eta_{i}=1 ; \operatorname{deg} \xi_{i}=1 ; \operatorname{deg}_{\text {Lie }}=\operatorname{deg}-2$

$r=1: \operatorname{deg} \xi_{1}=0, \operatorname{deg} \eta_{1}=\operatorname{deg} t=2$,

$\operatorname{deg} \xi_{2}=\operatorname{deg} \xi_{3}=\operatorname{deg} \eta_{2}=\operatorname{deg} \eta_{3}=1 ; \operatorname{deg}_{\text {Lie }}=\operatorname{deg}-2$

$r=3 \xi: \operatorname{deg} \xi_{i}=0, \operatorname{deg} \eta_{i}=\operatorname{deg} t=1 ; \operatorname{deg}_{\text {Lie }}=\operatorname{deg}-1$

$r=3 \eta: \operatorname{deg} \eta_{i}=0, \operatorname{deg} \xi_{i}=\operatorname{deg} t=1 ; \operatorname{deg}_{\text {Lie }}=\operatorname{deg}-1$

4) $\mathfrak{m b}(4 \mid 5 ; r)=(\mathfrak{a} \mathfrak{b}(4), \mathfrak{c v e c t}(0 \mid 3))_{*}^{m} \subset \mathfrak{m}(4), \quad r=0,1, K$

$r=0: \operatorname{deg} \tau=2, \operatorname{deg} u_{i}=\operatorname{deg} \xi_{i}=1$ for $i=0,1,2,3 ; \operatorname{deg}_{\text {Lie }}=\operatorname{deg}-2$

$r=1: \operatorname{deg} \tau=\operatorname{deg} \xi_{0}=\operatorname{deg} u_{1}=2, \operatorname{deg} u_{2}=\operatorname{deg} u_{3}=\operatorname{deg} \xi_{2}=\operatorname{deg} \xi_{3}=1 ;$

$\operatorname{deg} \xi_{1}=\operatorname{deg} u_{0}=0 ; \operatorname{deg}_{\text {Lie }}=\operatorname{deg}-2$

$r=K: \operatorname{deg} \tau=\operatorname{deg} \xi_{0}=3, \operatorname{deg} u_{0}=0, \operatorname{deg} u_{i}=2 ; \operatorname{deg} \xi_{i}=1$ for $i>0 ;$

$\operatorname{deg}_{\text {Lie }}=\operatorname{deg}-3$

5) $\mathfrak{k}_{\mathfrak{s l e}}(9 \mid 6 ; r)=\left(\mathfrak{h} \mathfrak{e i}(8 \mid 6), \mathfrak{s v e c t}_{3,4}(4)\right)_{*}^{k} \subset \mathfrak{k}(9 \mid 6), \quad r=0,2, K$

$r=0: \operatorname{deg} t=2, \operatorname{deg} p_{i}=\operatorname{deg} q_{i}=\operatorname{deg} \xi_{i}=\operatorname{deg} \eta_{i}=1 ; \operatorname{deg}_{\text {Lie }}=\operatorname{deg}-2$

$r=2: \operatorname{deg} t=\operatorname{deg} q_{3}=\operatorname{deg} q_{4}=\operatorname{deg} \eta_{1}=2$,

$\operatorname{deg} q_{1}=\operatorname{deg} q_{2}=\operatorname{deg} p_{1}=\operatorname{deg} p_{2}=\operatorname{deg} \eta_{2}=\operatorname{deg} \eta_{3}=\operatorname{deg} \zeta_{2}=\operatorname{deg} \zeta_{3}=1 ;$

$\operatorname{deg} p_{3}=\operatorname{deg} p_{4}=\operatorname{deg} \zeta_{1}=0 ; \operatorname{deg}_{L i e}=\operatorname{deg}-2$

$r=K: \operatorname{deg} t=\operatorname{deg} q_{i}=2, \operatorname{deg} p_{i}=0 ; \operatorname{deg} \zeta_{i}=\operatorname{deg} \eta_{i}=1 ; \operatorname{deg}_{L i e}=\operatorname{deg}-2$.

These names reflect the method of construction of these algebras, rather than their own properties. To name and understand these superalgebras adequately, their further interpretation is required. In parentheses stands the superdimension of the 
superspace of indeterminates on which the algebra is realized by vector fields; this realization is considered as a point of reference for regradings $r$. Since superdimensions of $\mathfrak{g}$ are distinct for the thirteen exceptional simple vectorial Lie superalgebras $\mathfrak{g}$, it is natural to call them briefly $\mathfrak{e}(5 \mid 4), \ldots, \mathfrak{e}(5 \mid 10)$ (see the table below) except for the first, $\mathfrak{s l e}(4 \mid 3)$.

More exactly, we consider simple filtered Lie superalgebras $\mathcal{L}$ with decreasing filtration of the form

$$
\mathcal{L}=\mathcal{L}_{-d} \supset \mathcal{L}_{-d+1} \supset \cdots \supset \mathcal{L}_{0} \supset \mathcal{L}_{1} \supset \ldots
$$

of finite depth $d$. The very term "filtered algebra" implies that $\left[\mathcal{L}_{i}, \mathcal{L}_{j}\right] \subset \mathcal{L}_{i+j}$ and we additionally require that

1) $\mathcal{L}_{0}$ is a maximal subalgebra of finite codimension;

2) the filtration is transitive: for any non-zero $x \in L_{k}$ for $k \geq 0$, where $L_{k}=$ $\mathcal{L}_{k} / \mathcal{L}_{k+1}$, there is $y \in L_{-1}$ such that $[x, y] \neq 0$.

Conditions 1) and 2) manifestly imply that $\operatorname{dim} L_{k}<\infty$ for all $k$ and the $\mathbb{Z}$ graded Lie superalgebra $L=\bigoplus_{k \geq-d} L_{k}$ associated with $\mathcal{L}$ grows polynomially, i.e., $\operatorname{dim} \bigoplus_{k \leq n} L_{k}$ grows as a polynomial in $n$. Such filtrations are called, after [W], Weisfeiler filtrations; we will shortly write W-filtrations and call the gradings associated with W-filtrations $W$-gradings. Since such filtered Lie superalgebras $\mathcal{L}$ (and associated with them graded ones, $L$ ) can be realized by vector fields with formal or polynomial coefficients, we refer to these Lie superalgebras as vectorial ones.

Any W-filtration can be considered as a basis of neighborhoods of zero in a topology, so the result can be read as the list of the exceptional simple complete vectorial Lie superalgebras. Thus, from the point of view of classification of the Wfiltered complete Lie superalgebras, there are five families of exceptional algebras consisting of 14 individual algebras. The algebras inside each family are isomorphic as abstract ones, but are distinct as filtered ones. Here are the corresponding first terms of the graded algebras (cf. sec. 0.8), where the sign $\boxplus$ (resp. $\in$ ) denotes the semidirect sum with the subspace or ideal on the left (right) of it:

\begin{tabular}{|c|c|c|c|c|}
\hline $\mathfrak{g}$ & $\mathfrak{g}_{-2}$ & $\mathfrak{g}_{-1}$ & $\mathfrak{g}_{0}$ & $\operatorname{dim} \mathfrak{g}_{-}$ \\
\hline $\mathfrak{v} \mathfrak{l} \mathfrak{e}(4 \mid 3)$ & - & $\Pi(\Lambda(3) / \mathbb{C} 1)$ & $\mathfrak{c}(\mathfrak{v e c t}(0 \mid 3))$ & $4 \mid 3$ \\
\hline $\mathfrak{v l e}(4 \mid 3 ; 1)$ & $\mathbb{C} \cdot 1$ & $\mathrm{id} \otimes \Lambda(2)$ & $\mathfrak{c}\left(\mathfrak{s l}(2) \otimes \Lambda(2) \boxplus T^{1 / 2}(\mathfrak{v e c t}(0 \mid 2))\right.$ & $5 \mid 4$ \\
\hline $\mathfrak{v} \mathfrak{l} \mathfrak{e}(4 \mid 3 ; K)$ & $\operatorname{id}(\mathfrak{s l}(3))$ & $\operatorname{id}(\mathfrak{s l}(3)) \otimes \operatorname{id}(\mathfrak{s l}(2)) \otimes 1$ & $\mathfrak{s l}(3) \oplus \mathfrak{s l}(2) \oplus \mathbb{C} z$ & $3 \mid 6$ \\
\hline $\mathfrak{v a s}(4 \mid 4)$ & - & spin & $\mathfrak{a} \mathfrak{s}$ & $4 \mid 4$ \\
\hline $\mathfrak{k a s}$ & $\mathbb{C} \cdot 1$ & id & $\mathfrak{c o}(6)$ & $1 \mid 6$ \\
\hline $\mathfrak{k a s}(; 1)$ & $\Lambda(1)$ & $\operatorname{id}(\mathfrak{s l}(2)) \otimes \operatorname{id}(\mathfrak{g l}(2)) \otimes \Lambda(1)$ & $(\mathfrak{s l}(2) \oplus \mathfrak{g l}(2)) \boxplus \mathfrak{v e c t}(0 \mid 1)$ & $5 \mid 5$ \\
\hline $\mathfrak{k} \mathfrak{a s}(; 3 \xi)$ & - & $\Lambda(3)$ & $\Lambda(3) \oplus \mathfrak{s l}(1 \mid 3)$ & $4 \mid 4$ \\
\hline $\mathfrak{k a s}(; 3 \eta)$ & - & $\operatorname{Vol}_{0}(0 \mid 3)$ & $\mathfrak{c}(\mathfrak{v e c t}(0 \mid 3))$ & $4 \mid 3$ \\
\hline $\mathfrak{m} \mathfrak{b}(4 \mid 5)$ & $\Pi(\mathbb{C} \cdot 1)$ & $\operatorname{Vol}(0 \mid 3)$ & $\mathfrak{c}(\mathfrak{v e c t}(0 \mid 3))$ & $4 \mid 5$ \\
\hline $\mathfrak{m b}(4 \mid 5 ; 1)$ & $\Lambda(2) / \mathbb{C} \cdot 1$ & $\mathrm{id} \otimes \Lambda(2)$ & $\mathfrak{c}\left(\mathfrak{s l}(2) \otimes \Lambda(2) \boxplus T^{1 / 2}(\mathfrak{v e c t}(0 \mid 2))\right.$ & $5 \mid 6$ \\
\hline $\mathfrak{m} \mathfrak{b}(4 \mid 5 ; K)$ & $\operatorname{id}(\mathfrak{s l}(3))$ & $\operatorname{id}(\mathfrak{s l}(3)) \otimes \operatorname{id}(\mathfrak{s l}(2)) \otimes 1$ & $\mathfrak{s l}(3) \oplus \mathfrak{s l}(2) \oplus \mathbb{C} z$ & $3 \mid 8$ \\
\hline $\mathfrak{k s l e}_{\mathfrak{s}}(9 \mid 6)$ & $\mathbb{C} \cdot 1$ & $\Pi\left(T_{0}^{0}(\overrightarrow{0})\right)$ & $\mathfrak{s v e c t}(0 \mid 4)_{3,4}$ & $9 \mid 6$ \\
\hline $\mathfrak{k} \mathfrak{s} \mathfrak{l}(9 \mid 6 ; 2)$ & $\operatorname{id}(\mathfrak{s l}(3 \mid 1))$ & $\operatorname{id}(\mathfrak{s l}(2)) \otimes \Lambda(3)$ & $(\mathfrak{s l}(2) \otimes \Lambda(3)) \boxplus \mathfrak{s l}(1 \mid 3)$ & $11 \mid 9$ \\
\hline $\mathfrak{k} \mathfrak{s l e}(9 \mid 6 ; K)$ & id & $\Lambda^{2}(\mathrm{id})$ & $\mathfrak{s l}(5)$ & $5 \mid 10$ \\
\hline
\end{tabular}

Observe that $\mathfrak{m} \mathfrak{b}(4 \mid 5 ; K)_{-3} \cong \Pi(\operatorname{id}(\mathfrak{s l}(2)))$, whereas in all the other cases $\mathfrak{g}_{-3}=0$. 
Observe that unlike the regradings of the series (see sec. 0.4) where the minimal realization is attained at $r=0$, some of the exceptional algebras have several minimal realizations.

The word "exceptional" implies that a classification is handy; indeed, for the detailed proof announced in [LSh1] and during a conference in honor of D. Buchsbaum (November 1997, Boston); see [LSh4], [LSh2].

The article is divided into several sections, according to the method of construction. Boring calculations are gathered in the appendices. The statements on simplicity are proved via Kac's criteria; cf. [K1].

Open problems. (1) Give more explicit geometric realizations of the exceptional Lie superalgebras (what structures do they preserve?).

(2) Certain exceptional Lie superalgebra are deformations of (nonsimple) Lie superalgebras whose brackets are easy to describe. In this paper the cocycle is described clumsily, in components of the generating functions. Describe the cocycle (i.e., the bracket itself) in terms of generating functions rather than their components. (An attempt is made in $[\mathrm{ShP}]$.)

(3) Find out what our exceptional Lie superalgebras add to the list of simple finite dimensional Lie algebras over an algebraically closed field of characteristic 2 via Leites' conjecture, either directly (cf. [L2], [KL]) or via Volichenko algebras ([LSe]).

Remark. The results of this paper and the related contribution to classification of the stringy superalgebras [GLS] (hep-th 9702120) were obtained in Stockholm in June 1996 and delivered at the seminar of E. Ivanov, JINR, Dubna (July, 1996), Voronezh winter school (Jan. 12-18, 1997). This paper was preprinted as hep-th 9702121, new $\S 7$ is added to it now; a brief description is also to appear in Russian in Functionalnyj Analiz i Prilozheniya.

\section{BACKGROUND}

0.1. Linear algebra in superspaces. Generalities. Superization has certain subtleties, often disregarded or expressed as in [L], [L3] or [M]; too briefly. We will dwell on them a bit.

A superspace is a $\mathbb{Z} / 2$-graded space; for a superspace $V=V_{\overline{0}} \oplus V_{\overline{1}}$ denote by $\Pi(V)$ another copy of the same superspace: with the shifted parity, i.e., $(\Pi(V))_{\bar{i}}=$ $V_{\bar{i}+\overline{1}}$. The superdimension of $V$ is $\operatorname{dim} V=p+q \varepsilon$, where $\varepsilon^{2}=1$ and $p=\operatorname{dim} V_{\overline{0}}$, $q=\operatorname{dim} V_{\overline{1}}$. (Usually $\operatorname{dim} V$ is expressed as a pair $(p, q)$ or $p \mid q$; this obscures the fact that $\operatorname{dim} V \otimes W=\operatorname{dim} V \cdot \operatorname{dim} W$ which is clear with the use of $\varepsilon$.)

A superspace structure in $V$ induces the superspace structure in the space $\operatorname{End}(V)$. A basis of a superspace is always a basis consisting of homogeneous vectors; let $\operatorname{Par}=\left(p_{1}, \ldots, p_{\operatorname{dim} V}\right)$ be an ordered collection of their parities. We call Par the format of the basis of $V$. A square supermatrix of format (size) Par is a $\operatorname{dim} V \times \operatorname{dim} V$ matrix whose $i$ th row and $i$ th column are of the same parity $p_{i}$. The matrix unit $E_{i j}$ is supposed to be of parity $p_{i}+p_{j}$ and the bracket of supermatrices (of the same format) is defined via Sign Rule:

if something of parity $p$ moves past something of parity $q$ the sign $(-1)^{p q}$ accrues; the formulas defined on homogeneous elements are extended to arbitrary ones via linearity. 
Examples of application of Sign Rule: setting $[X, Y]=X Y-(-1)^{p(X) p(Y)} Y X$ we get the notion of the supercommutator and the ensuing notions of the supercommutative superalgebra and the Lie superalgebra (that in addition to superskewcommutativity satisfies the super Jacobi identity, i.e., the Jacobi identity amended with the Sign Rule). The derivation of a superalgebra $A$ is a linear map $D: A \longrightarrow A$ such that it satisfies the Leibniz rule (and Sign rule)

$$
D(a b)=D(a) b+(-1)^{p(D) p(a)} a D(b) .
$$

In particular, let $A=\mathbb{K}[x]$ be the free supercommutative polynomial superalgebra in $x=\left(x_{1}, \ldots, x_{n}\right)$, where the superstructure is determined by the parities of the indeterminates: $p\left(x_{i}\right)=p_{i}$. Partial derivatives are defined (with the help of super Leibniz Rule) by the formulas

$$
\frac{\partial x_{i}}{\partial x_{j}}=\delta_{i, j}
$$

Clearly, the collection $\mathfrak{d e r} A$ of all superdifferentiations of $A$ is a Lie superalgebra whose elements are of the form

$$
\sum f_{i}(x) \frac{\partial}{\partial x_{i}}
$$

(We do not usually use the sign $\wedge$ for the wedge product of differential forms on supermanifolds: in what follows we assume that the exterior differential is odd and the differential forms constitute a supercommutative superalgebra; however, we sometimes keep using the sign $\wedge$ while working on manifolds in order not to deviate too far from conventional notations.)

Usually, Par is of the form $(\overline{0}, \ldots, \overline{0}, \overline{1}, \ldots, \overline{1})$. Such a format is called standard. In this paper we can do without nonstandard formats. But they are vital in the study of systems of simple roots that the reader might be interested in; besides, they are direct analogs of the nonstandard gradings we consider.

The general linear Lie superalgebra of all supermatrices of size Par is denoted by $\mathfrak{g l}(P a r)$; usually, $\mathfrak{g l}(\overline{0}, \ldots, \overline{0}, \overline{1}, \ldots, \overline{1})$ is abbreviated to $\mathfrak{g l}\left(\operatorname{dim} V_{\overline{0}} \mid \operatorname{dim} V_{\overline{1}}\right)$. Any matrix from $\mathfrak{g l}($ Par $)$ can be expressed as the sum of its even and odd parts; in the standard format this is the block expression:

$$
\left(\begin{array}{ll}
A & B \\
C & D
\end{array}\right)=\left(\begin{array}{cc}
A & 0 \\
0 & D
\end{array}\right)+\left(\begin{array}{cc}
0 & B \\
C & 0
\end{array}\right), \quad p\left(\left(\begin{array}{cc}
A & 0 \\
0 & D
\end{array}\right)\right)=\overline{0}, p\left(\left(\begin{array}{cc}
0 & B \\
C & 0
\end{array}\right)\right)=\overline{1} .
$$

The supertrace is the map $\mathfrak{g l}(\operatorname{Par}) \longrightarrow \mathbb{C},\left(A_{i j}\right) \mapsto \sum(-1)^{p_{i}} A_{i i}$. Since $\operatorname{str}[x, y]=$ 0 , the space of supertraceless matrices constitutes the special linear Lie subsuperalgebra $\mathfrak{s l}($ Par $)$.

Lie superalgebras that preserve bilinear forms: two types. To the linear map $F$ of superspaces there corresponds the dual map $F^{*}$ between the dual superspaces; if $A$ is the supermatrix corresponding to $F$ in a basis of the format Par, then to $F^{*}$ the supertransposed matrix $A^{\text {st }}$ corresponds:

$$
\left(A^{s t}\right)_{i j}=(-1)^{\left(p_{i}+p_{j}\right)\left(p_{i}+p(A)\right)} A_{j i} .
$$

The supermatrices $X \in \mathfrak{g l}($ Par $)$ such that

$$
X^{s t} B+(-1)^{p(X) p(B)} B X=0 \text { for a homogeneous matrix } B \in \mathfrak{g l}(\text { Par })
$$

constitute the Lie superalgebra $\mathfrak{a u t}(B)$ that preserves the bilinear form on $V$ with matrix $B$. 
Recall that the supersymmetry of the homogeneous form $\omega$ means that its matrix $B$ satisfies the condition $B^{u}=B$, where $B^{u}=\left(\begin{array}{cc}R^{t} & (-1)^{p(B)} T^{t} \\ (-1)^{p(B)} S^{t} & -U^{t}\end{array}\right)$ for the matrix $B=\left(\begin{array}{cc}R & S \\ T & U\end{array}\right)$. Similarly, skew-supersymmetry of $B$ means that $B^{u}=-B$.

Most popular canonical forms of the nondegenerate supersymmetric form are the ones whose supermatrices in the standard format are the following canonical ones, $B_{e v}$ or $B_{e v}^{\prime}$ :

$$
B_{e v}(m \mid 2 n)=\left(\begin{array}{cc}
1_{m} & 0 \\
0 & J_{2 n}
\end{array}\right), \quad \text { where } J_{2 n}=\left(\begin{array}{cc}
0 & 1_{n} \\
-1_{n} & 0
\end{array}\right)
$$

or

$$
B_{e v}^{\prime}(m \mid 2 n)=\left(\begin{array}{cc}
\operatorname{antidiag}(1, \ldots, 1) & 0 \\
0 & J_{2 n}
\end{array}\right) .
$$

The usual notation for $\mathfrak{a} \mathfrak{u t}\left(B_{e v}(m \mid 2 n)\right)$ is $\mathfrak{o s p}(m \mid 2 n)$ or $\mathfrak{o s p}^{s y}(m \mid 2 n)$.

Recall that the "upsetting" of forms $u: \operatorname{Bil}(V, W) \longrightarrow \operatorname{Bil}(W, V)$ becomes for $V=W$ an involution $u: B \mapsto B^{u}$. This involution separates symmetric and skew-symmetric forms. The passage from $V$ to $\Pi(V)$ sends the supersymmetric forms to superskew-symmetric ones, preserved by the "symplectico-orthogonal" Lie superalgebra $\mathfrak{o s p}^{s k}(m \mid 2 n)$ which is isomorphic to $\mathfrak{o s p}^{s y}(m \mid 2 n)$ but has a different matrix realization. We never use notation $\mathfrak{s p}^{\prime} \mathfrak{o}(2 n \mid m)$ in order not to confuse with the special Poisson superalgebra.

In the standard format the matrix realizations of these algebras are:

$$
\begin{gathered}
\mathfrak{o s p}(m \mid 2 n)=\left\{\left(\begin{array}{ccc}
E & Y & X^{t} \\
X & A & B \\
-Y^{t} & C & -A^{t}
\end{array}\right)\right\} ; \quad \mathfrak{o s p}^{s k}(m \mid 2 n)=\left\{\left(\begin{array}{ccc}
A & B & X \\
C & -A^{t} & Y^{t} \\
Y & -X^{t} & E
\end{array}\right)\right\}, \\
\text { where }\left(\begin{array}{cc}
A & B \\
C & -A^{t}
\end{array}\right) \in \mathfrak{s p}(2 n), \quad E \in \mathfrak{o}(m) \text { and }{ }^{t} \text { is the usual transposition. }
\end{gathered}
$$

A nondegenerate supersymmetric odd bilinear form $B_{\text {odd }}(n \mid n)$ can be reduced to the canonical form whose matrix in the standard format is $J_{2 n}$. A canonical form of the superskew odd nondegenerate form in the standard format is $\Pi_{2 n}=\left(\begin{array}{cc}0 & 1_{n} \\ 1_{n} & 0\end{array}\right)$. The usual notation for $\mathfrak{a u t}\left(B_{\text {odd }}(\operatorname{Par})\right)$ is $\mathfrak{p e}(\operatorname{Par})$. The passage from $V$ to $\Pi(V)$ sends the supersymmetric forms to superskew-symmetric ones and establishes an isomorphism $\mathfrak{p e}^{s y}($ Par $) \cong \mathfrak{p e}^{s k}($ Par $)$. This Lie superalgebra is called, as A. Weil suggested, periplectic. In the standard format these superalgebras are shorthanded as in the following formula, where their matrix realizations are also given:

$$
\begin{aligned}
& \mathfrak{p e}^{s y}(n)=\left\{\left(\begin{array}{cc}
A & B \\
C & -A^{t}
\end{array}\right), \text { where } B=-B^{t}, C=C^{t}\right\} ; \\
& \mathfrak{p e}^{s k}(n)=\left\{\left(\begin{array}{cc}
A & B \\
C & -A^{t}
\end{array}\right), \text { where } B=B^{t}, C=-C^{t}\right\} .
\end{aligned}
$$

The special periplectic superalgebra is $\mathfrak{s p e}(n)=\{X \in \mathfrak{p} \mathfrak{e}(n): \operatorname{str} X=0\}$.

Observe that though the Lie superalgebras $\mathfrak{o s p}^{s y}(m \mid 2 n)$ and $\mathfrak{p e}^{s k}(2 n \mid m)$, as well as $\mathfrak{p e}^{s y}(n)$ and $\mathfrak{p e}^{s k}(n)$, are isomorphic, the difference between them is sometimes crucial, see Remark 0.6 below. 
0.2. Vectorial Lie superalgebras. The standard realization. The elements of $\mathcal{L}=\mathfrak{d e r} \mathbb{C}[[u]]$ are considered as vector fields. The Lie algebra $\mathcal{L}$ has only one maximal subalgebra $\mathcal{L}_{0}$ of finite codimension (consisting of the fields that vanish at the origin). The subalgebra $\mathcal{L}_{0}$ determines a filtration of $\mathcal{L}$ : set

$$
\mathcal{L}_{-1}=\mathcal{L} \quad \text { and } \quad \mathcal{L}_{i}=\left\{D \in \mathcal{L}_{i-1}:[D, \mathcal{L}] \subset \mathcal{L}_{i-1}\right\} \text { for } i \geq 1 .
$$

The associated graded Lie algebra $L=\bigoplus_{i \geq-1} L_{i}$, where $L_{i}=\mathcal{L}_{i} / \mathcal{L}_{i+1}$, consists of the vector fields with polynomial coefficients.

Suppose $\mathcal{L}_{0} \subset \mathcal{L}$ is a maximal subalgebra of finite codimension and $\mathcal{L}_{0}$ contains no ideals of $\mathcal{L}$. For the Lie algebra $\mathcal{L}=\mathfrak{d e r} \mathbb{C}[u]$ the minimal nontrivial $\mathcal{L}_{0^{-}}$ submodule of $\mathcal{L}$ containing $\mathcal{L}_{0}$ coincides with $\mathcal{L}$. This is not so for superalgebras; not all subalgebras $\mathcal{L}$ of $\mathfrak{d e r} \mathbb{C}[u, \xi]$ have this property. Let $\mathcal{L}_{-1}$ be a minimal subspace of $\mathcal{L}$ containing $\mathcal{L}_{0}$, different from $\mathcal{L}_{0}$ and $\mathcal{L}_{0}$-invariant. Construct a filtration of $\mathcal{L}$ by setting for $i \geq 1$ :

$$
\mathcal{L}_{-i-1}=\left[\mathcal{L}_{-1}, \mathcal{L}_{-i}\right]+\mathcal{L}_{-i} \quad \text { and } \quad \mathcal{L}_{i}=\left\{D \in \mathcal{L}_{i-1}:\left[D, \mathcal{L}_{-1}\right] \subset \mathcal{L}_{i-1}\right\} .
$$

Since the codimension of $\mathcal{L}_{0}$ is finite, the filtration takes the form

$$
\mathcal{L}=\mathcal{L}_{-d} \supset \ldots \mathcal{L}_{0} \supset \ldots
$$

for some $d$. This $d$ is called the depth of $\mathcal{L}$ or of the associated graded Lie superalgebra $L$.

Considering the subspaces (0.2.3) as the basis of a topology, we can complete the graded or filtered Lie superalgebras $L$ or $\mathcal{L}$; the elements of the completion are the vector fields with formal power series as coefficients. Though the structure of the graded algebras is easier to describe, in applications the completed Lie superalgebras are usually needed.

Unlike Lie algebras, simple vectorial superalgebras possess several maximal subalgebras of finite codimension. We will describe them, together with the corresponding gradings, in sec. 0.4.

1) General algebras. Let $x=\left(u_{1}, \ldots, u_{n}, \theta_{1}, \ldots, \theta_{m}\right)$, where the $u_{i}$ are even indeterminates and the $\theta_{j}$ are odd ones. The Lie superalgebra $\mathfrak{v e c t}(n \mid m)$ consists of superdifferentiations of $\mathfrak{d e r} \mathbb{C}[x]$; it is called the general vectorial superalgebra.

2) Special algebras. The divergence of the field $D=\sum_{i} f_{i} \frac{\partial}{\partial u_{i}}+\sum_{j} g_{j} \frac{\partial}{\partial \theta_{j}}$ is the function (in our case: a polynomial, or a series)

$$
\operatorname{div} D=\sum_{i} \frac{\partial f_{i}}{\partial u_{i}}+\sum_{j}(-1)^{p\left(g_{j}\right)} \frac{\partial g_{i}}{\partial \theta_{j}} .
$$

- The Lie superalgebra $\mathfrak{s v e c t}(n \mid m)=\{D \in \mathfrak{v e c t}(n \mid m): \operatorname{div} D=0\}$ is called the special or divergence-free vectorial superalgebra. The notion of divergence depends on coordinates. Another description of $\mathfrak{s v e c t}$ is as follows:

$$
\mathfrak{s v e c t}(n \mid m)=\left\{D \in \mathfrak{v e c t}(n \mid m): L_{D} v l_{x}=0\right\},
$$

where $v^{2} l_{x}$ is the volume form with constant coefficients in coordinates $x$ and $L_{D}$ the Lie derivative with respect to $D$.

- The Lie superalgebra $\mathfrak{s v e c t}_{\lambda}(0 \mid m)=\left\{D \in \mathfrak{v e c t}(0 \mid m): \operatorname{div}\left(1+\lambda \theta_{1} \cdots \cdots \theta_{m}\right) D=0\right\}$ — the deform of $\mathfrak{s v e c t}(0 \mid m)$ — has no particular name and is also called the deformed special or deformed divergence-free vectorial superalgebra. Clearly, $\mathfrak{s v e c t}_{\lambda}(0 \mid m) \cong$ $\mathfrak{s v e c t}_{\mu}(0 \mid m)$ for $\lambda \mu \neq 0$. 
Observe that $p(\lambda) \equiv m(\bmod 2)$, i.e., for odd $m$ the parameter of deformation $\lambda$ is odd.

Remark. Sometimes we write $\operatorname{vect}(x)$ or even $\operatorname{vect}(V)$ if $V=\operatorname{Span}(x)$ and use similar notations for the subalgebras of $\mathfrak{v e c t}$ introduced below. Some algebraists sometimes abbreviate $\mathfrak{v e c t}(n)$ and $\mathfrak{s v e c t}(n)$ to $W_{n}$ (in honor of Witt) and $S_{n}$, respectively.

3) The algebras that preserve Pfaff equations and differential 2-forms.

- Set $u=\left(t, p_{1}, \ldots, p_{n}, q_{1}, \ldots, q_{n}\right)$; let

$$
\tilde{\alpha}_{1}=d t+\sum_{1 \leq i \leq n}\left(p_{i} d q_{i}-q_{i} d p_{i}\right)+\sum_{1 \leq j \leq m} \theta_{j} d \theta_{j} \quad \text { and } \quad \tilde{\omega}_{0}=d \tilde{\alpha}_{1} .
$$

The form $\tilde{\alpha}_{1}$ is called contact, the form $\tilde{\omega}_{0}$ is called symplectic.

Sometimes it is more convenient to redenote the $\theta$ 's and set

$$
\begin{aligned}
\xi_{j}=\frac{1}{\sqrt{2}}\left(\theta_{j}-i \theta_{r+j}\right) ; \quad \eta_{j}=\frac{1}{\sqrt{2}}\left(\theta_{j}+i \theta_{r+j}\right) \quad\left(\text { here } i^{2}=-1\right) \\
\text { for } j \leq r=[m / 2], \quad \theta=\theta_{2 r+1}
\end{aligned}
$$

and in place of $\tilde{\omega}_{0}$ or $\tilde{\alpha}_{1}$ take $\alpha$ and $\omega_{0}=d \alpha_{1}$, respectively, where

$$
\begin{aligned}
& \alpha_{1}=d t+\sum_{1 \leq i \leq n}\left(p_{i} d q_{i}-q_{i} d p_{i}\right)+\sum_{1 \leq j \leq r}\left(\xi_{j} d \eta_{j}+\eta_{j} d \xi_{j}\right) \quad \text { if } m=2 r \\
& \alpha_{1}=d t+\sum_{1 \leq i \leq n}\left(p_{i} d q_{i}-q_{i} d p_{i}\right)+\sum_{1 \leq j \leq r}\left(\xi_{j} d \eta_{j}+\eta_{j} d \xi_{j}\right)+\theta d \theta \quad \text { if } m=2 r+1 .
\end{aligned}
$$

The Lie superalgebra that preserves the Pfaff equation $\alpha_{1}=0$, i.e., the superalgebra

$$
\begin{aligned}
\mathfrak{k}(2 n+1 \mid m)=\{D \in \mathfrak{v e c t}(2 n+1 \mid m): & \left.L_{D} \alpha_{1}=f_{D} \alpha_{1}\right\} \\
& \text { for a polynomial } f_{D} \in \mathbb{C}[t, p, q, \theta]
\end{aligned}
$$

is called the contact superalgebra.

The Lie superalgebra $\mathfrak{p o}(2 n \mid m)$ that preserves not just the Pfaff equation determined by $\alpha_{1}$ but the form itself, i.e.,

$$
\mathfrak{p o}(2 n \mid m)=\left\{D \in \mathfrak{k}(2 n+1 \mid m): L_{D} \alpha_{1}=0\right\}
$$

is called the Poisson superalgebra. (A geometric interpretation of the Poisson superalgebra: it is the Lie superalgebra that preserves the connection with form $\alpha_{1}$ in the line bundle over a symplectic supermanifold with the symplectic form $d \alpha_{1}$.)

- Similarly, set $u=q=\left(q_{1}, \ldots, q_{n}\right)$, let $\theta=\left(\xi_{1}, \ldots, \xi_{n} ; \tau\right)$ be odd. Set

$$
\alpha_{0}=d \tau+\sum_{i}\left(\xi_{i} d q_{i}+q_{i} d \xi_{i}\right), \quad \omega_{1}=d \alpha_{0}
$$

and call these forms the odd-contact and, as A. Weil suggested, periplectic, respectively.

The Lie superalgebra that preserves the Pfaff equation $\alpha_{0}=0$, i.e., the superalgebra

$\mathfrak{m}(n)=\left\{D \in \mathfrak{v e c t}(n \mid n+1): L_{D} \alpha_{0}=f_{D} \cdot \alpha_{0}\right.$ for a polynomial $\left.f_{D} \in \mathbb{C}[q, \xi, \tau]\right\}$

is called the odd-contact superalgebra.

The Lie superalgebra

$$
\mathfrak{b}(n)=\left\{D \in \mathfrak{m}(n): L_{D} \alpha_{0}=0\right\}
$$


is called the Buttin superalgebra ([L3]). (A geometric interpretation of the Buttin superalgebra: it is the Lie superalgebra that preserves the connection with form $\alpha_{1}$ in the line bundle of rank $\varepsilon$ over a periplectic supermanifold, i.e., the supermanifold with the periplectic form $d \alpha_{0}$.)

The Lie superalgebras

$$
\mathfrak{s m}(n)=\{D \in \mathfrak{m}(n): \operatorname{div} D=0\}, \mathfrak{s} \mathfrak{b}(n)=\{D \in \mathfrak{b}(n): \operatorname{div} D=0\}
$$

are called the divergence-free (or special) odd-contact and special Buttin superalgebras, respectively.

Remark. A relation with finite dimensional geometry is as follows. Clearly, $\operatorname{ker} \alpha_{1}=$ ker $\tilde{\alpha}_{1}$. The restriction of $\omega_{0}$ to ker $\alpha_{1}$ is the orthosymplectic form $B_{e v}(m \mid 2 n)$; the restriction of $\omega_{0}$ to ker $\tilde{\alpha}_{1}$ is $B_{e v}^{\prime}(m \mid 2 n)$. Similarly, the restriction of $\omega_{1}$ to $\operatorname{ker} \alpha_{0}$ is the periplectic form $B_{\text {odd }}(n \mid n)$.

0.3 . Generating functions. A laconic way to describe the elements of $\mathfrak{k}, \mathfrak{m}$ and their subalgebras is via generating functions.

- Odd form $\alpha_{1}$ or $\tilde{\alpha}_{1}$. For $f \in \mathbb{C}[t, p, q, \theta]$ set:

$$
K_{f}=(2-E)(f) \frac{\partial}{\partial t}-H_{f}+\frac{\partial f}{\partial t} E,
$$

where $E=\sum_{i} y_{i} \frac{\partial}{\partial y_{i}}$ (here the $y$ are all the coordinates except $t$ ) is the Euler operator (which counts the degree with respect to the $y$ ), and $H_{f}$ is the hamiltonian field with Hamiltonian $f$ that preserves $d \tilde{\alpha}_{1}$ :

$$
H_{f}=\sum_{i \leq n}\left(\frac{\partial f}{\partial p_{i}} \frac{\partial}{\partial q_{i}}-\frac{\partial f}{\partial q_{i}} \frac{\partial}{\partial p_{i}}\right)-(-1)^{p(f)}\left(\sum_{j \leq m} \frac{\partial f}{\partial \theta_{j}} \frac{\partial}{\partial \theta_{j}}\right) .
$$

The choice of the form $\alpha_{1}$ instead of $\tilde{\alpha}_{1}$ only affects the form of $H_{f}$ that we give for $m=2 k+1$ :

$$
H_{f}=\sum_{i \leq n}\left(\frac{\partial f}{\partial p_{i}} \frac{\partial}{\partial q_{i}}-\frac{\partial f}{\partial q_{i}} \frac{\partial}{\partial p_{i}}\right)-(-1)^{p(f)}\left(\sum_{j \leq k}\left(\frac{\partial f}{\partial \xi_{j}} \frac{\partial}{\partial \eta_{j}}+\frac{\partial f}{\partial \eta_{j}} \frac{\partial}{\partial \xi_{j}}\right)+\frac{\partial f}{\partial \theta} \frac{\partial}{\partial \theta}\right) .
$$

- Even form $\alpha_{0}$. For $f \in \mathbb{C}[q, \xi, \tau]$ set:

$$
M_{f}=(2-E)(f) \frac{\partial}{\partial \tau}-L e_{f}-(-1)^{p(f)} \frac{\partial f}{\partial \tau} E,
$$

where $E=\sum_{i} y_{i} \frac{\partial}{\partial y_{i}}$ (here the $y$ are all the coordinates except $\tau$ ) is the Euler operator, and

$$
L e_{f}=\sum_{i \leq n}\left(\frac{\partial f}{\partial q_{i}} \frac{\partial}{\partial \xi_{i}}+(-1)^{p(f)} \frac{\partial f}{\partial \xi_{i}} \frac{\partial}{\partial q_{i}}\right)
$$

Since

$$
L_{K_{f}}\left(\alpha_{1}\right)=2 \frac{\partial f}{\partial t} \alpha_{1}, \quad L_{M_{f}}\left(\alpha_{0}\right)=-(-1)^{p(f)} 2 \frac{\partial f}{\partial \tau} \alpha_{0},
$$

it follows that $K_{f} \in \mathfrak{k}(2 n+1 \mid m)$ and $M_{f} \in \mathfrak{m}(n)$. Observe that

$$
p\left(L e_{f}\right)=p\left(M_{f}\right)=p(f)+\overline{1} \text {. }
$$


- To the supercommutators $\left[K_{f}, K_{g}\right]$ or $\left[M_{f}, M_{g}\right]$ there correspond contact brackets of the generating functions:

$$
\left[K_{f}, K_{g}\right]=K_{\{f, g\}_{k . b .}} ; \quad\left[M_{f}, M_{g}\right]=M_{\{f, g\}_{m . b .}} .
$$

The explicit formulas for the contact brackets are as follows. Let us first define the brackets on functions that do not depend on $t$ (resp. $\tau$ ).

The Poisson bracket $\{\cdot, \cdot\}_{P . b}$. (in the realization with the form $\tilde{\omega}_{0}$ ) is given by the formula

$$
\{f, g\}_{P . b .}=\sum_{i \leq n}\left(\frac{\partial f}{\partial p_{i}} \frac{\partial g}{\partial q_{i}}-\frac{\partial f}{\partial q_{i}} \frac{\partial g}{\partial p_{i}}\right)-(-1)^{p(f)} \sum_{j \leq m} \frac{\partial f}{\partial \theta_{j}} \frac{\partial g}{\partial \theta_{j}} \text { for } f, g \in \mathbb{C}[p, q, \theta]
$$

and in the realization with the form $\omega_{0}$ for $m=2 k+1$ it is given by the formula

$$
\begin{aligned}
& \{f, g\}_{P . b .}=\sum_{i \leq n}\left(\frac{\partial f}{\partial p_{i}} \frac{\partial g}{\partial q_{i}}-\frac{\partial f}{\partial q_{i}} \frac{\partial g}{\partial p_{i}}\right) \\
& -(-1)^{p(f)}\left(\sum_{j \leq k}\left(\frac{\partial f}{\partial \xi_{j}} \frac{\partial g}{\partial \eta_{j}}+\frac{\partial f}{\partial \eta_{j}} \frac{\partial g}{\partial \xi_{j}}\right)+\frac{\partial f}{\partial \theta} \frac{\partial g}{\partial \theta}\right) \text { for } f, g \in \mathbb{C}[p, q, \xi, \eta, \theta] .
\end{aligned}
$$

The Buttin bracket $\{\cdot, \cdot\}_{B . b}$. is given by the formula

$$
\{f, g\}_{\text {B.b. }}=\sum_{i \leq n}\left(\frac{\partial f}{\partial q_{i}} \frac{\partial g}{\partial \xi_{i}}+(-1)^{p(f)} \frac{\partial f}{\partial \xi_{i}} \frac{\partial g}{\partial q_{i}}\right) \text { for } f, g \in \mathbb{C}[q, \xi] .
$$

Remark. What we call here the Buttin bracket was discovered in pre-super era by Schouten. Buttin was the first to observe that the Schouten bracket determines a Lie superalgebra; Leites interpreted it in terms of mechanic ([L1]) and Batalin with Vilkovisky later rediscovered this mechanic with much success, see [GPS]. The Schouten bracket was originally defined on the superspace of multivector fields on a manifold, i.e., on the superspace $\Gamma\left(\Lambda^{\bullet}(T(M))\right) \cong \Lambda_{\mathcal{F}}(\operatorname{Vect}(M))$ of sections of the exterior algebra (over the algebra $\mathcal{F}$ of functions) of the tangent bundle. The explicit formula (in which the hatted slot should be ignored, as usual) of the Schouten bracket is

$$
\begin{gathered}
{\left[X_{1} \wedge \cdots \wedge \cdots \wedge X_{k}, Y_{1} \wedge \cdots \wedge Y_{l}\right]} \\
=\sum_{i, j}(-1)^{i+j}\left[X_{i}, Y_{j}\right] \wedge X_{1} \wedge \cdots \wedge \hat{X}_{i} \wedge \cdots \wedge X_{k} \wedge Y_{1} \wedge \cdots \wedge \hat{Y}_{j} \wedge \cdots \wedge Y_{l} .
\end{gathered}
$$

With the help of Sign Rule we easily superize formula $(*)$ for the case when manifold $M$ is replaced with supermanifold $\mathcal{M}$. Let $x$ and $\xi$ be the even and odd coordinates on $\mathcal{M}$. Setting $\theta_{i}=\Pi\left(\partial x_{i}\right)=\check{x}_{i}, q_{j}=\Pi\left(\partial \xi_{j}\right)=\check{\xi}_{j}$ we get an identification of the Schouten bracket of vector fields on $\mathcal{M}$ with the Buttin bracket of functions on the supermanifold $\check{\mathcal{M}}$ whose coordinates are $x, \xi$ and $\check{x}, \check{\xi}$; the transformation of $x, \xi$ induces that of the checked coordinates. (Physicists call the checked variables ghosts; cf. [GPS].)

In terms of the Poisson and Buttin brackets, respectively, the contact brackets take the form

$$
\{f, g\}_{k . b .}=(2-E)(f) \frac{\partial g}{\partial t}-\frac{\partial f}{\partial t}(2-E)(g)-\{f, g\}_{P . b .}
$$

and, respectively,

$$
\{f, g\}_{m . b .}=(2-E)(f) \frac{\partial g}{\partial \tau}+(-1)^{p(f)} \frac{\partial f}{\partial \tau}(2-E)(g)-\{f, g\}_{B . b .} .
$$


The Lie superalgebras of Hamiltonian fields (or Hamiltonian superalgebra) and its special subalgebra (defined only if $n=0$ ) are

$$
\mathfrak{h}(2 n \mid m)=\left\{D \in \mathfrak{v e c t}(2 n \mid m): L_{D} \omega_{0}=0\right\}
$$

and

$$
\mathfrak{s h}(m)=\left\{H_{f} \in \mathfrak{h}(0 \mid m): \int \text { vol }_{\theta}=0\right\} .
$$

Its odd analogues are the Lie superalgebra of Leitesian fields introduced in [L1] and its special subalgebra:

$$
\mathfrak{l}(n)=\left\{D \in \mathfrak{v e c t}(n \mid n): L_{D} \omega_{1}=0\right\} \text { and } \mathfrak{s l e}(n)=\{D \in \mathfrak{l e}(n): \operatorname{div} D=0\} .
$$

It is not difficult to prove the following isomorphisms (as superspaces):

$$
\begin{aligned}
\mathfrak{k}(2 n+1 \mid m) & \cong \operatorname{Span}\left(K_{f}: f \in \mathbb{C}[t, p, q, \xi]\right) ; \\
\mathfrak{h}(2 n \mid m) & \cong \operatorname{Span}\left(H_{f}: f \in \mathbb{C}[p, q, \theta]\right) ; \\
\mathfrak{m}(n) & \cong \operatorname{Span}\left(M_{f}: f \in \mathbb{C}[\tau, q, \xi]\right) ; \\
\mathfrak{l}(n) & \cong \operatorname{Span}\left(L_{f}: f \in \mathbb{C}[q, \xi]\right) .
\end{aligned}
$$

Remark. 1) It is obvious that the Lie superalgebras of the series $\mathfrak{v e c t}, \mathfrak{s v e c t}, \mathfrak{h}$ and $\mathfrak{p o}$ for $n=0$ are finite dimensional.

2) A Lie superalgebra of the series $\mathfrak{h}$ is the quotient of the Lie superalgebra po modulo the one-dimensional center $\mathfrak{z}$ generated by constant functions.

Similarly, $\mathfrak{l}$ and $\mathfrak{s l e}$ are the quotients of $\mathfrak{b}$ and $\mathfrak{s} \mathfrak{b}$, respectively, modulo the one-dimensional (odd) center $\mathfrak{z}$ generated by constant functions.

Set $\mathfrak{s p o}(m)=\left\{K_{f} \in \mathfrak{p o}(0 \mid m): \int f \operatorname{vol}_{\xi}=0\right\}$ and $\mathfrak{s h}(m)=\mathfrak{s p o}(m) / \mathbb{C} \cdot K_{1}$.

Since, as is easy to see ([GLS]), $\operatorname{div} K_{f}=(2 n+2-m) \frac{\partial f}{\partial t}$ for $K_{f} \in \mathfrak{k}(2 n+1 \mid m)$, the divergence-free subalgebra of $\mathfrak{k}(2 n+1 \mid m)$ is either the algebra itself (for $m=2 n+2$ ) or its Poisson subalgebra. Nothing new.

The "odd" counterpart is more interesting. Since

$$
\operatorname{div} M_{f}=(-1)^{p(f)} 2\left((1-E) \frac{\partial f}{\partial \tau}-\sum_{i \leq n} \frac{\partial^{2} f}{\partial q_{i} \partial \xi_{i}}\right),
$$

we can define the divergence-free subalgebra of $\mathfrak{m}(n)$ :

$$
\mathfrak{s m}(n)=\operatorname{Span}\left(M_{f} \in \mathfrak{m}(n):(1-E) \frac{\partial f}{\partial \tau}=\sum_{i \leq n} \frac{\partial^{2} f}{\partial q_{i} \partial \xi_{i}}\right) .
$$

In particular,

$$
\operatorname{div} L e_{f}=(-1)^{p(f)} 2 \sum_{i \leq n} \frac{\partial^{2} f}{\partial q_{i} \partial \xi_{i}} .
$$

(The odd analog of the Laplacian, namely, the operator

$$
\Delta=\sum_{i \leq n} \frac{\partial^{2}}{\partial q_{i} \partial \xi_{i}}
$$

on a periplectic supermanifold appeared in physics under the name of BRST operator; cf. [GPS]. The divergence-free vector fields from $\mathfrak{s l e}(n)$ are generated by harmonic functions, i.e., such that $\Delta(f)=0$.) 
Lie superalgebras $\mathfrak{s l e}(n), \mathfrak{s b}(n)$ and $\mathfrak{s v e c t}(1 \mid n)$ have ideals $\mathfrak{s l e}^{\circ}(n), \mathfrak{s b}^{\circ}(n)$ and $\mathfrak{s v e c t} \mathfrak{t}^{\circ}(n)$ of codimension 1 (more exactly, $\varepsilon^{n-1}$ ) defined from the exact sequences

$$
\begin{gathered}
0 \longrightarrow \mathfrak{s l e}^{\circ}(n) \longrightarrow \mathfrak{s l e}(n) \longrightarrow \mathbb{C} \cdot L e_{\xi_{1} \ldots \xi_{n}} \longrightarrow 0, \\
0 \longrightarrow \mathfrak{s b}^{\circ}(n) \longrightarrow \mathfrak{s b}(n) \longrightarrow \mathbb{C} \cdot M_{\xi_{1} \ldots \xi_{n}} \longrightarrow 0, \\
0 \longrightarrow \mathfrak{s v e c t}^{\circ}(1 \mid n) \longrightarrow \mathfrak{s v e c t}(1 \mid n) \longrightarrow \mathbb{C} \cdot \xi_{1} \ldots \xi_{n} \frac{\partial}{\partial t} \longrightarrow 0 .
\end{gathered}
$$

0.4. Nonstandard realizations. In [LSh4] we classified the nonstandard gradings of the simple vectorial Lie superalgebras. Here are the main points. Clearly, the gradings in the series $\mathfrak{v e c t}$ induce the gradings in the series $\mathfrak{s v e c t}$, and $\mathfrak{s v e c t}{ }^{\circ}$; the gradings in $\mathfrak{m}$ induce the gradings in $\mathfrak{s m}, \mathfrak{l e}, \mathfrak{s l e}, \mathfrak{s l e} \mathfrak{e}^{\circ}, \mathfrak{b}, \mathfrak{s b}, \mathfrak{s} \mathfrak{b}^{\circ}$; the gradings in $\mathfrak{k}$ induce the gradings in $\mathfrak{p o}, \mathfrak{h}$. In what follows we consider $\mathfrak{k}(2 n+1 \mid m)$ as preserving the Pfaff equation $\alpha=0$, where

$$
\alpha=d t+\sum_{i \leq n}\left(p_{i} d q_{i}-q_{i} d p_{i}\right)+\sum_{j \leq r}\left(\xi_{j} d \eta_{j}+\eta_{j} d \xi_{j}\right)+\sum_{k \geq m-2 r} \theta_{k} d \theta_{k} .
$$

The standard realizations are marked by $(*)$ and in this case indication to $r=$ 0 is omitted; note that (bar several exceptions for small $m, n$ ) it corresponds to the case of the minimal codimension of $\mathcal{L}_{0}$. Observe that the Lie superalgebras corresponding to different values of $r$ are isomorphic as abstract Lie superalgebras, but as filtered ones they are distinct.

\begin{tabular}{|c|c|}
\hline Lie superalgebra & its $\mathbb{Z}$-grading \\
\hline $\mathfrak{v e c t}(n \mid m ; r)$, & $\operatorname{deg} u_{i}=\operatorname{deg} \xi_{j}=1$ for any $i, j$ \\
\cline { 2 - 3 } $0 \leq r \leq m$ & $\operatorname{deg} \xi_{j}=0$ for $1 \leq j \leq r ;$ \\
& $\operatorname{deg} u_{i}=\operatorname{deg} \xi_{r+s}=1$ for any $i, s$ \\
\hline \multirow{3}{*}{$\mathfrak{m}(n ; r)}$, & $\operatorname{deg} \tau=2, \operatorname{deg} q_{i}=\operatorname{deg} \xi_{i}=1$ for any $i$ \\
\cline { 2 - 3 } $0 \leq r \leq n$ & $\operatorname{deg} \tau=\operatorname{deg} q_{i}=1, \operatorname{deg} \xi_{i}=0$ for any $i$ \\
\cline { 2 - 3 } & $\operatorname{deg} \tau=\operatorname{deg} q_{i}=2, \operatorname{deg} \xi_{i}=0$ for $1 \leq i \leq r<n ;$ \\
\hline $\mathfrak{k}(2 n+1 \mid m ; r)$, & $\operatorname{deg} u_{r+j}=\operatorname{deg} \xi_{r+j}=1$ for any $j$ \\
\hline \multirow{3}{*}{$0 \leq r \leq\left[\frac{m}{2}\right]$} & $\operatorname{deg} t=2$, \\
\cline { 2 - 3 } & $\operatorname{deg} p_{i}=\operatorname{deg} q_{i}=\operatorname{deg} \xi_{j}=\operatorname{deg} \eta_{j}=\operatorname{deg} \theta_{k}=1$ for any $i, j, k$ \\
\hline $\mathfrak{k}(1 \mid 2 m ; m)$ & $\operatorname{deg} t=\operatorname{deg} \xi_{i}=2, \operatorname{deg} \eta_{i}=0$ for $1 \leq i \leq r \leq\left[\frac{m}{2}\right] ;$ \\
\hline
\end{tabular}

Observe that $\mathfrak{k}(1 \mid 2 ; 1) \cong \mathfrak{v e c t}(1 \mid 1)$ and $\mathfrak{m}(1 ; 1) \cong \mathfrak{v e c t}(1 \mid 1)$ as filtered Lie superalgebras.

The exceptional nonstandard gradings. Observe immediately that though these regradings are not Weisfeiler ones they are used all the time in string theories.

Denote the indeterminates and their respective exceptional degrees as follows (here $\mathfrak{k}(1 \mid 2)$ is considered in the realization that preserves the Pfaff equation $\alpha_{1}=0$ ):

\begin{tabular}{|r|c|c|c|c|}
\hline & & $\mathfrak{v e c t}(1 \mid 1)$ & $\mathfrak{k}(1 \mid 2)$ & $\mathfrak{m}(1)$ \\
\hline $\mathfrak{v e c t}(1 \mid 1)$ & $t, \xi$ & 1,1 & 2,1 & $1,-1$ \\
\hline $\mathfrak{k}(1 \mid 2)$ & $t, \xi, \eta$ & $1,1,0$ & $2,1,1$ & $1,2,-1$ \\
\hline $\mathfrak{m}(1)$ & $\tau, q, \xi$ & $1,1,0$ & $1,2,-1$ & $2,1,1$ \\
\hline
\end{tabular}


Denote the nonstandard exceptional realizations by indicating the above degrees after a semicolon. In addition to the isomorphisms indicated above we get the following isomorphisms of the filtered Lie superalgebras:

$$
\begin{aligned}
\mathfrak{v e c t}(1 \mid 1 ; 2,1) \cong \mathfrak{k}(1 \mid 2) ; & \mathfrak{k}(1 \mid 2 ; 1,2,-1) \cong \mathfrak{m}(1) ; \\
\mathfrak{v e c t}(1 \mid 1 ; 1,-1) \cong \mathfrak{m}(1) ; & \mathfrak{m}(1 ; 1,2,-1) \cong \mathfrak{k}(1 \mid 2) .
\end{aligned}
$$

0.5. Cartan prolongs. We will repeatedly make use of Cartan's prolongation; see [St]. So let me recall the definition and generalize it somewhat. Let $\mathfrak{g}$ be a Lie algebra, $V$ a $\mathfrak{g}$-module, $S^{i}$ the operator of the $i$ th symmetric power. Set $\mathfrak{g}_{-1}=V$, $\mathfrak{g}_{0}=\mathfrak{g}$ and define the $i$ th Cartan prolong for $i>0$ as

$$
\begin{aligned}
\mathfrak{g}_{i} & =\left\{X \in \operatorname{Hom}\left(\mathfrak{g}_{-1}, \mathfrak{g}_{i-1}\right): X\left(v_{0}\right)\left(v_{1}, v_{2}, \ldots v_{i}\right)\right. \\
& \left.=X\left(v_{1}\right)\left(v_{0}, v_{2}, \ldots, v_{i}\right) \text { for any } v_{i} \in \mathfrak{g}_{-1}\right\} .
\end{aligned}
$$

Equivalently, let $i: S^{k+1}\left(\mathfrak{g}_{-1}\right)^{*} \otimes \mathfrak{g}_{-1} \longrightarrow S^{k}\left(\mathfrak{g}_{-1}\right)^{*} \otimes \mathfrak{g}_{-1}^{*} \otimes \mathfrak{g}_{-1}$ be a natural embedding and $j: S^{k}\left(\mathfrak{g}_{-1}\right)^{*} \otimes \mathfrak{g}_{0} \longrightarrow S^{k}\left(\mathfrak{g}_{-1}\right)^{*} \otimes \mathfrak{g}_{-1}^{*} \otimes \mathfrak{g}_{-1}$ a natural map. Then $\mathfrak{g}_{k}=i\left(S^{k+1}\left(\mathfrak{g}_{-1}\right)^{*} \otimes \mathfrak{g}_{-1}\right) \cap j\left(S^{k}\left(\mathfrak{g}_{-1}\right)^{*} \otimes \mathfrak{g}_{0}\right)$.

The sum $\left(\mathfrak{g}_{-1}, \mathfrak{g}_{0}\right)_{*}=\bigoplus_{i>-1} \mathfrak{g}_{i}$ is called the Cartan prolong (the result of Cartan's prolongation) of the pair $(V, \mathfrak{g})$. (In what follows • in superscript denotes, as is now customary, the collection of all degrees, while $*$ is reserved for dualization; in the subscripts we retain the old fashioned $*$ instead of $\cdot$ to avoid too close a contact with the punctuation marks.)

Suppose that the $\mathfrak{g}_{0}$-module $\mathfrak{g}_{-1}$ is faithful. Then, clearly,

$$
\begin{aligned}
& \left(\mathfrak{g}_{-1}, \mathfrak{g}_{0}\right)_{*} \subset \mathfrak{v e c t}(n)=\mathfrak{d e r} \mathbb{C}\left[x_{1}, \ldots, x_{n}\right] \text {, where } n=\operatorname{dim} \mathfrak{g}_{-1} \text { and } \\
& \qquad \mathfrak{g}_{i}=\left\{D \in \mathfrak{v e c t}(n): \operatorname{deg} D=i,[D, X] \in \mathfrak{g}_{i-1} \text { for any } X \in \mathfrak{g}_{-1}\right\} .
\end{aligned}
$$

It is subject to an easy verification that the Lie algebra structure on $\mathfrak{v e c t}(n)$ induces same on $\left(\mathfrak{g}_{-1}, \mathfrak{g}_{0}\right)_{*}$.

Of the four simple vectorial Lie algebras, three are Cartan prolongs:

$$
\mathfrak{v e c t}(n)=(\mathrm{id}, \mathfrak{g l}(n))_{*}, \quad \mathfrak{s v e c t}(n)=(\mathrm{id}, \mathfrak{s l}(n))_{*} \quad \text { and } \quad \mathfrak{h}(2 n)=(\mathrm{id}, \mathfrak{s p}(n))_{*} .
$$

The fourth one $-\mathfrak{k}(2 n+1)-$ is also the prolong under a trifle more general construction described as follows.

1) The generalized Cartan prolong. Let $\mathfrak{g}_{-}=\bigoplus_{-d<i<-1} \mathfrak{g}_{i}$ be a nilpotent $\mathbb{Z}$-graded Lie algebra and $\mathfrak{g}_{0} \subset \mathfrak{d} \mathfrak{e} \mathfrak{r}_{0} \mathfrak{g}$ a Lie subalgebra of the $\mathbb{Z}$-grading-preserving derivations. For $k>0$ define the $k$ th prolong of the pair $\left(\mathfrak{g}_{-}, \mathfrak{g}_{0}\right)$ to be

$$
\mathfrak{g}_{k}=\left(j\left(S^{\bullet}\left(\mathfrak{g}_{-}\right)^{*} \otimes \mathfrak{g}_{0}\right) \cap i\left(S^{\bullet}\left(\mathfrak{g}_{-}\right)^{*} \otimes \mathfrak{g}_{-}\right)\right)_{k},
$$

where the subscript $k$ in the right hand side singles out the component of degree $k$.

Set $\left(\mathfrak{g}_{-}, \mathfrak{g}_{0}\right)_{*}=\bigoplus_{i \geq-d} \mathfrak{g}_{i} ;$ then, as is easy to verify, $\left(\mathfrak{g}_{-}, \mathfrak{g}_{0}\right)_{*}$ is a Lie algebra.

What is the Lie algebra of contact vector fields in these terms? Denote by $\mathfrak{h e i}(2 n)$ the Heisenberg Lie algebra: its space is $W \oplus \mathbb{C} \cdot z$, where $W$ is a $2 n$-dimensional space endowed with a nondegenerate skew-symmetric bilinear form $B$ and the bracket in $\mathfrak{h e i}(2 n)$ is given by the following conditions:

$$
z \text { is in the center and }[v, w]=B(v, w) \cdot z \text { for any } v, w \in W .
$$

Clearly, $\mathfrak{k}(2 n+1)$ is $(\mathfrak{h e i}(2 n), \mathfrak{c s p}(2 n))_{*}$, where for any $\mathfrak{g}$ we write $\mathfrak{c g}=\mathfrak{g} \oplus \mathbb{C} \cdot z$ or $\mathfrak{c}(\mathfrak{g})$ to denote the trivial central extension with the 1-dimensional even center generated by $z$. 
2) The partial Cartan prolong. The Cartan prolongation $\left(\mathfrak{g}_{-1}, \mathfrak{g}_{0}\right)_{*}$ starts with nonpositive elements and is completely determined by them. Define the partial Cartan prolongation with a part of $\mathfrak{g}_{1}$. Over $\mathbb{C}$, this construction is a purely super phenomenon but in the study of Lie algebras over fields of prime characteristic it was independently observed.

Take a $\mathfrak{g}_{0}$-submodule $\mathfrak{h}_{1}$ in $\mathfrak{g}_{1}$. Suppose $\left[\mathfrak{g}_{-1}, \mathfrak{h}_{1}\right]=\mathfrak{g}_{0}$, not a proper subalgebra of $\mathfrak{g}_{0}$. Define the second prolongation of $\left(\bigoplus_{i \leq 0} \mathfrak{g}_{i}, \mathfrak{h}_{1}\right)$ to be $\mathfrak{h}_{2}=\left\{D \in \mathfrak{g}_{2}:\left[D, \mathfrak{g}_{-1}\right] \subset\right.$ $\left.\mathfrak{h}_{1}\right\}$. The terms $\mathfrak{h}_{i}$ for $i>2$ are similarly defined: $\mathfrak{h}_{i}=\left\{D \in \mathfrak{g}_{i}:\left[D, \mathfrak{g}_{-1}\right] \subset \mathfrak{h}_{i-1}\right\}$. Set $\mathfrak{h}_{i}=\mathfrak{g}_{i}$ for $i \leq 0$ and $\mathfrak{h}_{*}=\sum \mathfrak{h}_{i}$.

Example. $\operatorname{vect}(1 \mid n ; n)$ is a subalgebra of $\mathfrak{k}(1 \mid 2 n ; n)$. The former is obtained as Cartan's prolong of the same nonpositive part as $\mathfrak{k}(1 \mid 2 n ; n)$ and a submodule of $\mathfrak{k}(1 \mid 2 n ; n)_{1}$. The simple exceptional superalgebra $\mathfrak{k} \mathfrak{a} \mathfrak{s}$ introduced in $\S 3$ is another example.

To see the difference with the conventional Lie algebra case, consider $\mathfrak{v e c t}(m)_{1}$. As $\operatorname{vect}(m)_{0}=\mathfrak{g l}(m)$-module, it has two components, one, $\mathfrak{g}_{1}^{\prime}$, is isomorphic to $\mathfrak{v e c t}(m \mid n)_{-1}^{*}$, the other one, $\mathfrak{g}_{1}^{\prime \prime}$, consists of divergence-free fields and is isomorphic to $\mathfrak{s v e c t}(m)_{1}$. If we take $\mathfrak{g}_{1}^{\prime}$, then its partial Cartan prolongation terminates at once $\left(\mathfrak{v e c t}(m)_{-} \oplus \mathfrak{g}_{1}^{\prime} \cong \mathfrak{s l}(m+1)\right)$; whereas $\left[\mathfrak{v e c t}(m)_{-1}, \mathfrak{g}_{1}^{\prime \prime}\right]=\mathfrak{s l}(m)$, a proper subalgebra of $\mathfrak{v e c t}(m)_{0}$. Clearly, the situation is the same for $\mathfrak{v e c t}(m \mid n)$.

0.6. Lie superalgebras of vector fields as the Cartan prolongs. The superization of the constructions from sec. 0.5 are straightforward: via Sign Rule. We thus get:

$$
\begin{gathered}
\mathfrak{v e c t}(m \mid n)=(\mathrm{id}, \mathfrak{g l}(m \mid n))_{*} ; \quad \mathfrak{s v e c t}(m \mid n)=(\operatorname{id}, \mathfrak{s l}(m \mid n))_{*} ; \\
\mathfrak{h}(2 m \mid n)=\left(\operatorname{id}, \mathfrak{o s p}^{s k}(m \mid 2 n)\right)_{*} ; \mathfrak{l}(n)=\left(\operatorname{id}, \mathfrak{p e}{ }^{s k}(n)\right)_{*} ; \quad \mathfrak{s l e}(n)=\left(\mathrm{id}, \mathfrak{s p e}^{s k}(n)\right)_{*} .
\end{gathered}
$$

Remark. Observe that the Cartan prolongs (id, $\left.\mathfrak{o s p}^{s y}(m \mid 2 n)\right)_{*}$ and (id, $\left.\mathfrak{p e}^{s y}(n)\right)_{*}$ are finite dimensional.

The generalized Cartan prolong of the pair $(\mathfrak{h e i}(2 n), \mathfrak{s p}(2 n))$ described in sec. 0.5 has, after superization, two analogs associated with the contact series $\mathfrak{k}$ and $\mathfrak{m}$, respectively.

- First, we define hei $(2 n \mid m)$ on the direct sum of a $(2 n, m)$-dimensional superspace $W$ endowed with a nondegenerate skew-symmetric bilinear form and a $(1,0)$-dimensional space spanned by $z$.

Clearly, we have $\mathfrak{k}(2 n+1 \mid m)=\left(\mathfrak{h e i}(2 n \mid m), \mathfrak{c}\left(\mathfrak{o s p}^{s k}(m \mid 2 n)\right)\right)_{*}$ and, given $\mathfrak{h e i}(2 n \mid m)$ and a subalgebra $\mathfrak{g}$ of $\mathfrak{c}\left(\mathfrak{o s p}^{s k}(m \mid 2 n)\right)$, we call $(\mathfrak{h} \mathfrak{e i}(2 n \mid m), \mathfrak{g})_{*}$ the $k$-prolong of $(W, \mathfrak{g})$, where $W$ is the identity $\mathfrak{c}\left(\mathfrak{o s p}^{s k}(m \mid 2 n)\right)$-module.

- The odd analog of $\mathfrak{k}$ is associated with the following odd analog of $\mathfrak{h} \mathfrak{e i}(2 n \mid m)$. Denote by $\mathfrak{a} \mathfrak{b}(n)$ the antibracket Lie superalgebra: its space is $W \oplus \mathbb{C} \cdot z$, where $W$ is an $n \mid n$-dimensional superspace endowed with a nondegenerate skew-symmetric odd bilinear form $B$; the bracket in $\mathfrak{a} \mathfrak{b}(n)$ is given by the following formulas:

$$
z \text { is odd and lies in the center; }[v, w]=B(v, w) \cdot z \text { for } v, w \in W \text {. }
$$

Set $\mathfrak{m}(n)=\left(\mathfrak{a} \mathfrak{b}(n), \mathfrak{c}\left(\mathfrak{p} \mathfrak{e}^{s k}(n)\right)\right)_{*}$ and, given $\mathfrak{a} \mathfrak{b}(n)$ and a subalgebra $\mathfrak{g}$ of $\mathfrak{c}\left(\mathfrak{p} \mathfrak{e}^{s k}(n)\right)$, we call $(\mathfrak{a} \mathfrak{b}(n), \mathfrak{g})_{*}$ the $m$-prolong of $(W, \mathfrak{g})$, where $W$ is the identity $\mathfrak{c}\left(\mathfrak{p} \mathfrak{e}^{s k}(n)\right)$ module. 
Generally, given a nondegenerate form $B$ on a superspace $W$ and a superalgebra $\mathfrak{g}$ that preserves $B$, we refer to the above generalized prolongations as to $m k$ prolongation of the pair $(W, \mathfrak{g})$.

0.7. Deformations of the Buttin superalgebras and $\mathfrak{v e c t}(m \mid n)$-modules. Here we reproduce a result of Kotchetkoff [Ko1] with some corrections (cf. [Ko2], [L3] and [LSh2]).

To consider the deformations, recall the definition of the $\mathfrak{v e c t}(m \mid n)$-module of tensor fields of type $V$; see [BL]. Let $V$ be the $\mathfrak{g l}(m \mid n)=\mathfrak{v e c t}_{0}(m \mid n)$-module with the lowest weight $\lambda$. Make $V$ into a $\mathfrak{g}_{\geq}$-module, where $\mathfrak{g}=\mathfrak{v e c t}(m \mid n)$ and $\mathfrak{g}_{\geq}=\bigoplus_{i \geq 0} \mathfrak{g}_{i}$, setting $\mathfrak{g}_{+} \cdot V=0$ for $\mathfrak{g}_{+}=\bigoplus_{i>0} \mathfrak{g}_{i}$. Let us realize $\mathfrak{g}$ by vector fields on the $m \mid n$-dimensional linear complex supermanifold $\mathcal{C}^{m \mid n}$ with coordinates $x$. The superspace $T(V)=\operatorname{Hom}_{U\left(\mathfrak{g}_{+}\right)}(U(\mathfrak{g}), V)$ is isomorphic, due to the PoincaréBirkhoff-Witt theorem, to $\mathbb{C}[[x]] \otimes V$. Its elements have a natural interpretation as formal tensor fields of type $V$. When $\lambda=(a, \ldots, a)$ we will simply write $T(\vec{a})$ instead of $T(\lambda)$.

Example. $T(\overrightarrow{0})$ is the superspace of functions; $\operatorname{Vol}(m \mid n)=T(1, \ldots, 1 ;-1, \ldots,-1)$ (the semicolon separates the first $m$ coordinates of the weight with respect to the matrix units $E_{i i}$ of $\left.\mathfrak{g l}(m \mid n)\right)$ is the superspace of densities or volume forms. We denote the generator of $\operatorname{Vol}(m \mid n)$ corresponding to the ordered set of coordinates $x$ by $\operatorname{vol}(x)$ or $\operatorname{vol}_{x}$. The space of $\lambda$-densities is $\operatorname{Vol}^{\lambda}(m \mid n)=T(\lambda, \ldots, \lambda ;-\lambda, \ldots,-\lambda)$. In particular, $\operatorname{Vol}^{\lambda}(m \mid 0)=T(\vec{\lambda})$ but $\operatorname{Vol}^{\lambda}(0 \mid n)=T(\overrightarrow{-\lambda})$. Over $\mathfrak{v e c t}(0 \mid n)$, we further set $V_{o l}=\left\{v \in T(\overrightarrow{-1}): \int v=0\right\}$ and $T_{0}(\overrightarrow{0})=T_{0}(\overrightarrow{0}) / \mathbb{C} \cdot 1 ;$ by definition, $V o l \cong T(\overrightarrow{0})$ over $\mathfrak{s w e c t}(0 \mid n)$, so we can set $T_{0}^{0}(\overrightarrow{0})=\operatorname{Vol}_{0} / \mathbb{C} \cdot 1$.

As is clear from the definition of the Buttin bracket, there is a regrading (namely, $\mathfrak{b}(n ; n)$ given by $\operatorname{deg} \xi_{i}=0, \operatorname{deg} q_{i}=1$ for all $\left.i\right)$ under which $\mathfrak{b}(n)$, initially of depth 2 , takes the form $\mathfrak{g}=\bigoplus_{i>-1} \mathfrak{g}_{i}$ with $\mathfrak{g}_{0}=\mathfrak{v e c t}(0 \mid n)$ and $\mathfrak{g}_{-1} \cong \Pi(\mathbb{C}[\xi])$.

Let us replace the $\mathfrak{v e c t}(0 \mid n)$-module $\mathfrak{g}_{-1}$ of functions (with inverted parity) with the module of $\lambda$-densities, i.e., set $\mathfrak{g}_{-1} \cong \mathbb{C}[\xi]\left(\operatorname{vol}_{\xi}\right)^{\lambda}$, where

$$
L_{D}\left(\operatorname{vol}_{\xi}\right)^{\lambda}=\lambda \operatorname{div} D \cdot \operatorname{vol}_{\xi}^{\lambda} \text { and } p\left(v^{\prime} l_{\xi}\right)^{\lambda}=\overline{1} .
$$

Then $\mathfrak{b}_{\lambda}(n ; n)$ — the Cartan's prolong $\left(\mathfrak{g}_{-1}, \mathfrak{g}_{0}\right)_{*}=\left(\Pi\left(\operatorname{Vol}(0 \mid n)^{\lambda}\right), \mathfrak{v e c t}(0 \mid n)\right)_{*}$ - is a deform of $\mathfrak{b}(n ; n)$. The collection of these deforms for various $\lambda \in \mathbb{C}$ constitutes a deformation of $\mathfrak{b}(n ; n)$; we called it the main deformation; see [ALSh]. (Though main, this deformation is not the quantization of the Buttin bracket; for the latter see [Ko1] or [L3].) The deform $\mathfrak{b}_{\lambda}(n)$ of $\mathfrak{b}(n)$ is the regrading of $\mathfrak{b}_{\lambda}(n ; n)$ inverse to the regrading of $\mathfrak{b}(n)$ into $\mathfrak{b}(n ; n)$.

Another description of the main deformation is as follows. Set

$$
\mathfrak{b}_{a, b}(n)=\left\{M_{f} \in \mathfrak{m}(n): a \operatorname{div} M_{f}=(-1)^{p(f)} 2(a-b n) \frac{\partial f}{\partial \tau}\right\} .
$$

It is subject to a direct check that $\mathfrak{b}_{a, b}(n ; n) \cong \mathfrak{b}_{\lambda}(n ; n)$ for $\lambda=\frac{2 a}{n(a-b)}$. This isomorphism shows that $\lambda$ actually runs over $\mathbb{C P}^{1}$, not $\mathbb{C}$. Observe that for $a=n b$, i.e., for $\lambda=\frac{2}{n-1}$, we have $\mathfrak{b}_{n b, b}(n) \cong \mathfrak{s m}(n)$.

As follows from the description of $\mathfrak{v e c t}(m \mid n)$-modules ([BL]) and the criteria for simplicity of $\mathbb{Z}$-graded Lie superalgebras $([\mathrm{K} 1])$, the Lie superalgebras $\mathfrak{b}_{\lambda}(n)$ are simple for $n>1$ and $\lambda \neq 0,-1$. The same criteria also make it clear that the $\mathfrak{b}_{\lambda}(n)$ are nonisomorphic for distinct $\lambda$ 's. (Notice, that at some values of $\lambda$ the Lie 
superalgebras $\mathfrak{b}_{\lambda}(n)$ have additional deformations distinct from the above. These deformations are partly described in [Ko1], [L3].)

The geometric interpretation of $\mathfrak{b}_{a, b}(n)$ follows from (0.7): this is the Lie superalgebra that preserves $\operatorname{vol}_{q, \xi, \tau}^{a} \alpha_{0}^{a-b n}$. The meaning of parameters $a$ and $b$ is clear from column $\mathfrak{g}_{0}$ in row $\mathfrak{b}_{a, b}(n)$ in Table 0.8 .

0.8. Several first terms that determine the Cartan and $m k$-prolongations. To facilitate the comparison of various vectorial superalgebras, consider the following table. The central element $z \in \mathfrak{g}_{0}$ is supposed to be chosen so that it acts on $\mathfrak{g}_{k}$ as $\left.k \cdot \mathrm{id}\right|_{\mathfrak{g}_{k}}$. As in Introduction, the sign $\boxplus$ (resp. $\notin$ ) denotes the semidirect sum with the subspace or ideal on the left (right) of it; $\Lambda(r)=\mathbb{C}\left[\xi_{1}, \ldots, \xi_{r}\right]$ is the Grassmann superalgebra.

\begin{tabular}{|c|c|c|c|}
\hline $\mathfrak{g}$ & $\mathfrak{g}_{-2}$ & $\mathfrak{g}_{-1}$ & $\mathfrak{g}_{0}$ \\
\hline \hline $\mathfrak{v e c t}(n \mid m ; r)$ & - & $\operatorname{id} \otimes \Lambda(r)$ & $\mathfrak{g l}(n \mid m-r) \otimes \Lambda(r) \nexists \mathfrak{v e c t}(0 \mid r)$ \\
\hline $\mathfrak{v e c t}(1 \mid m ; m)$ & - & $\Lambda(m)$ & $\Lambda(m) \nexists \mathfrak{v e c t}(0 \mid m)$ \\
\hline \hline $\mathfrak{s v e c t}(n \mid m ; r)$ & - & $\operatorname{id} \otimes \Lambda(r)$ & $\mathfrak{s l}(n \mid m-r) \otimes \Lambda(r) \nexists \mathfrak{v e c t}(0 \mid r)$ \\
\hline $\mathfrak{s v e c t}(1 \mid m ; m)$ & - & $\operatorname{Vol}(0 \mid m)$ & $\mathfrak{v e c t}(0 \mid m)$ \\
\hline $\mathfrak{s} \mathfrak{v e c t} \mathfrak{o}^{\circ}(1 \mid m ; m)$ & - & $\operatorname{Vol}_{0}(0 \mid m)$ & $\mathfrak{v e c t}(0 \mid m)$ \\
\hline $\mathfrak{s v e c t}^{\circ}(1 \mid 2)$ & - & $T_{0}(\overrightarrow{0})$ & $\mathfrak{v e c t}(0 \mid 2) \cong \mathfrak{s l}(1 \mid 2)$ \\
\hline $\mathfrak{s} \mathfrak{v e c t}(2 \mid 1)$ & - & $\Pi\left(T_{0}(\overrightarrow{0})\right)$ & $\mathfrak{v e c t}(0 \mid 2) \cong \mathfrak{s l}(2 \mid 1)$ \\
\hline
\end{tabular}

\begin{tabular}{|c|c|c|c|}
\hline $\mathfrak{k}(2 n+1 \mid m ; r)$ & $\Lambda(r)$ & $\mathrm{id} \otimes \Lambda(r)$ & $\mathfrak{c o s p}(m-2 r \mid 2 n) \otimes \Lambda(r) \nexists \mathfrak{v e c t}(0 \mid r)$ \\
\hline $\mathfrak{h}(2 n \mid m ; r)$ & $\Lambda(r) / \mathbb{C} \cdot 1$ & $\mathrm{id} \otimes \Lambda(r)$ & $\mathfrak{o s p}(m-2 r \mid 2 n) \otimes \Lambda(r) \nexists \mathfrak{v e c t}(0 \mid r)$ \\
\hline $\mathfrak{k}(1 \mid 2 m ; m)$ & - & $\Lambda(m)$ & $\Lambda(m) \nexists \mathfrak{v e c t}(0 \mid m)$ \\
\hline $\mathfrak{k}(1 \mid 2 m+1 ; m)$ & $\Lambda(m)$ & $\Pi(\Lambda(m))$ & $\Lambda(m) \nexists \mathfrak{v e c t}(0 \mid m)$ \\
\hline
\end{tabular}

Recall that $\mathfrak{b}_{a, b}(n) \cong \mathfrak{b}_{\lambda}(n)$ for $\lambda=\frac{2 a}{n(a-b)} ; z$ is the center (unit matrix) in $\mathfrak{g}_{0}, d$ is an outer derivation — the grading operator — of $\mathfrak{g}_{0}$.

\begin{tabular}{|c|c|c|c|}
\hline $\mathfrak{g}$ & $\mathfrak{g}_{-2}$ & $\mathfrak{g}_{-1}$ & $\mathfrak{g}_{0}$ \\
\hline \hline $\mathfrak{b}_{a, b}(n ; r)$ & $\Pi(\Lambda(r))$ & $\operatorname{id} \otimes \Lambda(r)$ & $(\mathfrak{s p e}(n-r) \boxplus \mathbb{C}(a z+b d)) \otimes \Lambda(r) \nexists \mathfrak{v e c t}(0 \mid r)$ \\
\hline $\mathfrak{b}_{\lambda}(n ; n)$ & - & $\Pi\left(\operatorname{Vol}^{\lambda}(0 \mid n)\right)$ & $\mathfrak{v e c t}(0 \mid n)$ \\
\hline \hline $\mathfrak{m}(n ; r)$ & $\Pi(\Lambda(r))$ & $\operatorname{id} \otimes \Lambda(r)$ & $\mathfrak{c p e}(n-r) \otimes \Lambda(r) \boxplus \mathfrak{v e c t}(0 \mid r)$ \\
\hline $\mathfrak{m}(n ; n)$ & - & $\Pi(\Lambda(n))$ & $\Lambda(n) \nexists \mathfrak{v e c t}(0 \mid n)$ \\
\hline \hline $\mathfrak{l} \mathfrak{e}(n ; r)$ & $\Pi(\Lambda(r)) / \mathbb{C} \cdot 1$ & $\operatorname{id} \otimes \Lambda(r)$ & $\mathfrak{p e}(n-r) \otimes \Lambda(r) \boxplus \mathfrak{v e c t}(0 \mid r)$ \\
\hline $\mathfrak{l} \mathfrak{e}(n ; n)$ & - & $\Pi\left(T_{0}(\overrightarrow{0})\right)$ & $\mathfrak{v e c t}(0 \mid n)$ \\
\hline \hline $\mathfrak{s l e}{ }^{\circ}(n ; r)$ & $\Pi(\Lambda(r)) / \mathbb{C} \cdot 1$ & $\operatorname{id} \otimes \Lambda(r)$ & $\mathfrak{s p e}(n-r) \otimes \Lambda(r) \boxplus \mathfrak{v e c t}(0 \mid r)$ \\
\hline $\mathfrak{s l e} \mathfrak{e}^{\circ}(n ; n)$ & - & $\Pi\left(T_{0}(\overrightarrow{0})\right)$ & $\mathfrak{s v e c t}(0 \mid n)$ \\
\hline
\end{tabular}




\section{The exceptional Lie superalgebra $\mathfrak{v a} \mathfrak{a}(4 \mid 4)=(\operatorname{spin}, \mathfrak{a} \mathfrak{s})_{*}$}

1.1. A. Sergeev's extension. Let $\omega$ be a nondegenerate superskew-symmetric odd bilinear form on an $(n, n)$-dimensional superspace $V$. In the standard basis of $V$ (all the even vectors come first) the canonical matrix of the form $\omega$ is $\left(\begin{array}{cc}0 & 1_{n} \\ 1_{n} & 0\end{array}\right)$ and the elements of $\mathfrak{p e}(n)=\mathfrak{a} \mathfrak{u}(\omega)$ can be represented by supermatrices of the form $\left(\begin{array}{cc}a & b \\ c & -a^{t}\end{array}\right)$, where $b=b^{t}, c=-c^{t}$. The Lie superalgebra $\mathfrak{s p e}(n)$ is singled out by the requirement that $\operatorname{tr} a=0$. Setting

$$
\operatorname{deg}\left(\left(\begin{array}{ll}
0 & 0 \\
c & 0
\end{array}\right)\right)=-1, \quad \operatorname{deg}\left(\left(\begin{array}{cc}
a & 0 \\
0 & -a^{t}
\end{array}\right)\right)=0, \quad \operatorname{deg}\left(\left(\begin{array}{ll}
0 & b \\
0 & 0
\end{array}\right)\right)=1,
$$

we endow $\mathfrak{p e}(n)$ with a $\mathbb{Z}$-grading. It is known $([\mathrm{K} 1])$ that $\mathfrak{s p e}(n)=\mathfrak{p e}(n) \cap \mathfrak{s l}(n \mid n)$ is a simple Lie superalgebra for $n \geq 3$.

A. Sergeev proved (1977, unpublished) that there exists just one nontrivial central extension of $\mathfrak{s p e}(n)$. It exists for $n=4$ and is denoted by as. Let us represent an arbitrary element $A \in \mathfrak{a s}$ as a pair $A=x+d \cdot z$, where $x \in \mathfrak{s p e}(4), d \in \mathbb{C}$ and $z$ is the central element. In the matrix form the bracket in $\mathfrak{a s}$ is

$$
\begin{aligned}
& {\left[\left(\begin{array}{cc}
a & b \\
c & -a^{t}
\end{array}\right)+d \cdot z,\left(\begin{array}{cc}
a^{\prime} & b^{\prime} \\
c^{\prime} & -a^{\prime t}
\end{array}\right)+d^{\prime} \cdot z\right]} \\
& \quad=\left[\left(\begin{array}{cc}
a & b \\
c & -a^{t}
\end{array}\right),\left(\begin{array}{cc}
a^{\prime} & b^{\prime} \\
c^{\prime} & -a^{\prime t}
\end{array}\right)\right]-\frac{1}{2} \operatorname{tr} c \tilde{c}^{\prime} \cdot z,
\end{aligned}
$$

where for the skew-symmetric matrix $c_{i j}=E_{i j}-E_{j i}$ we set $\tilde{c}_{i j}=c_{k l}$ for the even permutation (1234) $\mapsto(i j k l)$. Clearly, $\operatorname{deg} z=-2$ with respect to the grading (1.1).

1.2. The Lie superalgebra $\mathfrak{a} \mathfrak{s}$ can also be described with the help of the spinor representation. Consider $\mathfrak{p o}(0 \mid 6)$, the Lie superalgebra whose superspace is the Grassmann superalgebra $\Lambda(\xi, \eta)$ generated by $\xi_{1}, \xi_{2}, \xi_{3}, \eta_{1}, \eta_{2}, \eta_{3}$ and the bracket is the Poisson bracket. Recall that $\mathfrak{h}(0 \mid 6)=\operatorname{Span}\left(H_{f}: f \in \Lambda(\xi, \eta)\right)$.

Now, observe that $\mathfrak{s p e}(4)$ can be embedded into $\mathfrak{h}(0 \mid 6)$. Indeed, setting $\operatorname{deg} \xi_{i}=$ $\operatorname{deg} \eta_{i}=1$ for all $i$ we introduce a $\mathbb{Z}$-grading on $\Lambda(\xi, \eta)$ which, in turn, induces a $\mathbb{Z}$-grading on $\mathfrak{h}(0 \mid 6)$ of the form $\mathfrak{h}(0 \mid 6)=\bigoplus_{i \geq-1} \mathfrak{h}(0 \mid 6)_{i}$. Since $\mathfrak{s l}(4) \cong \mathfrak{o}(6)$, we can identify $\mathfrak{s p e}(4)_{0}$ with $\mathfrak{h}(0 \mid 6)_{0}$.

It is not difficult to see that the elements of degree -1 in $\mathfrak{s p e}(4)$ and $\mathfrak{h}(0 \mid 6)$ constitute isomorphic $\mathfrak{s l}(4) \cong \mathfrak{o}(6)$-modules. It is subject to a direct verification that it is possible to embed $\mathfrak{s p e}(4)_{1}$ into $\mathfrak{h}(0 \mid 6)_{1}$.

Sergeev's extension $\mathfrak{a} \mathfrak{s}$ is the result of the restriction on $\mathfrak{s p e}(4) \subset \mathfrak{h}(0 \mid 6)$ of the cocycle that turns $\mathfrak{h}(0 \mid 6)$ into $\mathfrak{p o}(0 \mid 6)$. The quantization deforms $\mathfrak{p o}(0 \mid 6)$ into $\mathfrak{g l}(\Lambda(\xi))$; the through maps $T_{\lambda}: \mathfrak{a s} \longrightarrow \mathfrak{p o}(0 \mid 6) \longrightarrow \mathfrak{g l}(\Lambda(\xi))$ are representations of $\mathfrak{a} \mathfrak{s}$ in the $4 \mid 4$-dimensional modules $\operatorname{spin}_{\lambda}$ distinct for distinct values $\lambda$ of the central element $z$. (Here $\lambda \in \mathbb{C}$ plays the role of Planck's constant.) The explicit form of $T_{\lambda}$ is as follows:

$$
T_{\lambda}:\left(\begin{array}{cc}
a & b \\
c & -a^{t}
\end{array}\right)+d \cdot z \mapsto\left(\begin{array}{cc}
a & b-\lambda \tilde{c} \\
c & -a^{t}
\end{array}\right)+\lambda d \cdot 1_{4 \mid 4},
$$

where $1_{4 \mid 4}$ is the unit matrix and $\tilde{c}$ is defined after formula (1.2). Clearly, $T_{\lambda}$ is an irreducible representation. 
1.3. Theorem. 1) The Cartan prolong $\mathfrak{f}^{\lambda}=\left(\operatorname{spin}_{\lambda}, \mathfrak{a s}\right)_{*}$ is infinite dimensional and simple for $\lambda \neq 0$.

2) $\mathfrak{f}^{\lambda} \cong \mathfrak{f}^{\mu}$ if $\lambda \cdot \mu \neq 0$.

Observe that though the representations $T_{\lambda}$ are distinct for $\lambda \neq 0$, the corresponding Cartan prolongs are isomorphic.

Convention. For brevity, we denote the isomorphic superalgebras $\mathfrak{f}^{\lambda}=\left(\operatorname{spin}_{\lambda}, \mathfrak{a s}\right)_{*}$ for any $\lambda \neq 0$ by $\mathfrak{v a s}(4 \mid 4)=(\operatorname{spin}, \mathfrak{a s})_{*}$.

Proof. Heading 1) consists of two statements: a) (spin, as) $k \neq 0$ for all $k>0 ; \mathrm{b}$ ) the Lie superalgebra $\left(\operatorname{spin}_{\lambda}, \mathfrak{a s}\right)_{*}$ has no nontrivial $\mathbb{Z}$-graded ideals.

a) This follows from the fact that the elements $u_{i}^{k+1} \partial \xi_{i}$ belong to (spin, as) $)_{k}$ for any $k>0$ and any $i$ (to prove the statement it suffices to consider only one $i$ ).

b) Assume the contrary: let $\mathfrak{i}=\bigoplus_{k \geq-1} \mathfrak{i}_{k}$ be a nonzero ideal of $\mathfrak{h}=(\operatorname{spin}, \mathfrak{a} \mathfrak{s})_{*}$. Let $x \in \mathfrak{i}_{k}$ be a nonzero homogeneous element. Since $\mathfrak{g}=\mathfrak{v e c t}(4 \mid 4) \supset(\operatorname{spin}, \mathfrak{a} \mathfrak{s})_{*}$ is transitive, then the superspace $(k+1$ brackets)

$$
\left[\mathfrak{h}_{-1},\left[\mathfrak{h}_{-1}, \ldots,\left[\mathfrak{h}_{-1}, x\right] \ldots\right]=\left[\mathfrak{g}_{-1},\left[\mathfrak{g}_{-1}, \ldots,\left[\mathfrak{g}_{-1}, x\right] \ldots\right] \subset \mathfrak{i}_{-1}\right.\right.
$$

is a nonzero subspace of $\mathfrak{h}_{-1}$. Since $T_{\lambda}$ is irreducible, $\mathfrak{i}_{-1}=\mathfrak{h}_{-1}$. The Jacobi identity implies that $\left[\mathfrak{i}_{-1}, \mathfrak{h}_{1}\right] \subset \mathfrak{i}_{0}$ is an ideal of $\mathfrak{h}_{0}$.

But $\mathfrak{h}_{0}=\mathfrak{a} \mathfrak{s}$ has only one nontrivial ideal, the center. Since $\left[\mathfrak{i}_{-1}, \mathfrak{h}_{1}\right]=\left[\mathfrak{h}_{-1}, \mathfrak{h}_{1}\right]$ contains elements of the form $u_{i} \frac{\partial}{\partial \xi_{i}}$ for any $i$, which do not belong to the center, it follows that $\mathfrak{i}_{0}=\mathfrak{h}_{0}$. In particular, $\mathfrak{i}_{0}$ contains the element

$$
T_{\lambda}(z)=-\lambda \sum\left(u_{i} \frac{\partial}{\partial u_{i}}+\xi_{i} \frac{\partial}{\partial \xi_{i}}\right) .
$$

But $\left[T_{\lambda}(z), h\right]=-\lambda \cdot k \cdot h$ for any $h \in \mathfrak{h}_{k}$. Hence, $\mathfrak{i}=\mathfrak{h}$ and $\mathfrak{h}$ is simple.

2 ) follows from the fact that the nonpositive parts of $\mathfrak{f}^{\lambda}$ and $\mathfrak{f}^{\mu}$ are isomorphic.

1.4. A problem. For $\lambda=0$ the representation $T_{0}$ is not faithful and $T_{0}(\mathfrak{a s})=$ $\mathfrak{s p e}(4)$. The Cartan prolong of the pair (id, $\mathfrak{s p e}(4))$ is well-known: this is $\mathfrak{s l e}(4)$. Recall that we can realize $\mathfrak{l}(n)$ by the generating functions - the elements of $\mathbb{C}[u, \xi]$ — with the Buttin bracket. The subalgebra $\mathfrak{s l e}(n)$ is generated by harmonic functions, i.e., by functions that satisfy $\Delta(f)=0$, where $\Delta=\sum_{i=1}^{n} \frac{\partial^{2}}{\partial u_{i} \partial \xi_{i}}$. The exceptional Lie superalgebra (spin, $\mathfrak{a s})_{*}$ is a deform of $\mathfrak{s l e}(4) \boxplus \mathbb{C} \cdot \sum\left(u_{i} \frac{\partial}{\partial u_{i}}+\xi_{i} \frac{\partial}{\partial \xi_{i}}\right)$. An explicit expression of the corresponding cocycle is desirable: it will enable us to express the bracket in $(\operatorname{spin}, \mathfrak{a s})_{*}$ in terms of harmonic functions (plus one more element).

\section{AN EXPLICIT FORM OF THE VECTOR FIELDS FROM $\mathfrak{v a s}(4 \mid 4)=(\operatorname{spin}, \mathfrak{a} \mathfrak{s}) * \subset \mathfrak{s v e c t}(4 \mid 4)$}

Every element $D \in \mathfrak{v e c t}(4 \mid 4)$ is of the form $D=\sum_{i \leq 4}\left(P_{i} \frac{\partial}{\partial \xi_{i}}+Q_{i} \frac{\partial}{\partial u_{i}}\right)$, where $P_{i}, Q_{i} \in \mathbb{C}[u, \xi]$.

2.1. Lemma. The homogeneous (with respect to parity) vector field $D \in \mathfrak{v e c t}(4 \mid 4)$ belongs to $(\mathrm{spin}, \mathfrak{a s})_{*}$ if and only if it satisfies the following system of equations:

$$
\frac{\partial Q_{i}}{\partial u_{j}}+(-1)^{p(D)} \frac{\partial P_{j}}{\partial \xi_{i}}=0 \text { for any } i \neq j
$$




$$
\begin{gathered}
\frac{\partial Q_{i}}{\partial u_{i}}+(-1)^{p(D)} \frac{\partial P_{i}}{\partial \xi_{i}}=\frac{1}{2} \sum_{1 \leq j \leq 4} \frac{\partial Q_{j}}{\partial u_{j}} \text { for } i=1,2,3,4 ; \\
\frac{\partial Q_{i}}{\partial \xi_{j}}+\frac{\partial Q_{j}}{\partial \xi_{i}}=0 \text { for any } i, j ; \\
\frac{\partial P_{i}}{\partial u_{j}}-\frac{\partial P_{j}}{\partial u_{i}}=(-1)^{p(D)} \cdot \lambda \cdot\left(\frac{\partial Q_{k}}{\partial \xi_{l}}-\frac{\partial Q_{l}}{\partial \xi_{k}}\right) \\
\text { for any even permutation }\left(\begin{array}{cccc}
1 & 2 & 3 & 4 \\
i & j & k & l
\end{array}\right) .
\end{gathered}
$$

2.1.1. Remark. 1) Observe that the sum of the 4 equations (2.2) yields that $\operatorname{div} D=$ 0 , i.e., $(\operatorname{spin}, \mathfrak{a s}) * \subset \mathfrak{s v e c t}(4 \mid 4)$.

2) For $\lambda=0$ the system (2.1)-(2.4) singles out the superalgebra

$$
\left(\mathfrak{s l e}{ }^{\circ}(4) \boxplus \mathbb{C} \cdot \sum\left(u_{i} \frac{\partial}{\partial u_{i}}+\xi_{i} \frac{\partial}{\partial \xi_{i}}\right)\right) \boxplus \mathbb{C} \cdot \operatorname{Le}_{\xi_{1} \xi_{2} \xi_{3} \xi_{4}} .
$$

2.1.2. Remark. Actually, any Cartan prolongation is obtained as a solution of some system of differential equations with constant coefficients. For Lie algebras this fact is lucidly explained in [St]. The supercase is absolutely analogous.

Let $\mathfrak{g}_{-1}=V=\operatorname{Span}\left(\frac{\partial}{\partial x_{i}}, i=1, \ldots, n\right)$. Then any vector field $D=\sum f_{i}(x) \partial_{x_{i}}$ generates a linear operator $L_{D}: V \longrightarrow \mathfrak{v e c t}(V)-$ the Lie derivative $-L_{D}\left(\frac{\partial}{\partial x_{i}}\right)=$ $\left[D, \frac{\partial}{\partial x_{i}}\right]$. This operator is a tensor object determined by the matrix $P(D)=\left(P_{i, j}\right)$, where $P_{i, j}=(-1)^{p(D) p\left(x_{j}\right)} \frac{\partial f_{i}}{\partial x_{j}}$. If $D \in \mathfrak{v e c t}(V)_{0}$, then the matrix $P(D)$ is a numerical one and can be singled out from $\mathfrak{g l}(V)$ by a homogeneous linear system. The fact that any operator of the left adjoint action $\operatorname{ad}^{l}\left(\frac{\partial}{\partial x_{i}}\right)$ commutes with its right twin $\operatorname{ad}^{r}\left(\frac{\partial}{\partial x_{i}}\right)$ means that any vector field $D \in \mathfrak{v e c t}(V)$ belongs to the Cartan prolongation $(V, \mathfrak{g})_{*}$ if and only if the matrix $P(D)$ is a $\mathfrak{g}$-valued function on $V$.

Proof. Denote by $\mathfrak{g}^{\lambda}=\bigoplus_{i \geq-1} \mathfrak{g}_{i}^{\lambda}$ for $\lambda \neq 0$ the space of solutions of the system (2.1)-(2.4). Clearly, $\mathfrak{g}_{-1}^{\lambda} \cong \operatorname{vect}(4 \mid 4)_{-1}$. Let $D \in \mathfrak{g}_{0}^{\lambda}$. Then the matrix of the operator $D$ in its action on $\mathfrak{g}_{-1}^{\lambda}$ is of the form

$$
\begin{aligned}
& \left(\begin{array}{ll}
a & b \\
c & d
\end{array}\right), \text { where } a_{i j}=\frac{\partial Q_{i}}{\partial u_{j}}, \quad b_{i j}=\frac{\partial P_{i}}{\partial u_{j}}, \\
& c_{i j}=(-1)^{p(D)} \frac{\partial Q_{i}}{\partial \xi_{j}}, \quad d_{i j}=(-1)^{p(D)} \frac{\partial P_{i}}{\partial \xi_{j}} .
\end{aligned}
$$

Therefore, equations (2.1) and (2.2) mean that $a+d^{t}=\left(\frac{1}{2} \operatorname{tr} a\right) \cdot 1_{4}$, equations (2.3) that $c+c^{t}=0$, equations (2.4) that $b-b^{t}=\lambda\left(\tilde{c}-\tilde{c}^{t}\right)$. Set

$$
a_{0}=a-\left(\frac{1}{4} \operatorname{tr} a\right) \cdot 1_{4}, \quad d_{0}=d-\left(\frac{1}{4} \operatorname{tr} a\right) \cdot 1_{4} .
$$

Then

$$
a_{0}+d_{0}^{t}=a+d^{t}-\left(\frac{1}{2} \operatorname{tr} a\right) \cdot 1_{4}=0 ; \quad c+c^{t}=0 ; \quad b-b^{t}=\lambda\left(\tilde{c}-\tilde{c}^{t}\right) .
$$

Comparing formulas (2.5) with (1.3) we see that $\mathfrak{g}_{0}^{\lambda}$ coincides with the image of $\mathfrak{a s}$ under $T_{\lambda}$, i.e., with $(\operatorname{spin}, \mathfrak{a s})_{0}$. 
Set

$$
D_{u_{j}}(D)=\sum_{i \leq 4}\left(\frac{\partial P_{i}}{\partial u_{j}} \frac{\partial}{\partial \xi_{i}}+\frac{\partial Q_{i}}{\partial u_{j}} \frac{\partial}{\partial u_{i}}\right)
$$

and

$$
\tilde{D}_{\xi_{j}}(D)=(-1)^{p(D)} \sum_{i \leq 4}\left(\frac{\partial P_{i}}{\partial \xi_{j}} \frac{\partial}{\partial \xi_{i}}+\frac{\partial Q_{i}}{\partial \xi_{j}} \frac{\partial}{\partial u_{i}}\right) .
$$

The operators $D_{u_{j}}$ and $\tilde{D}_{\xi_{j}}$, clearly, commute with the $\mathfrak{g}_{-1}^{\lambda}$-action. Observe that the operators commute, not supercommute.

Since equations (2.1)-(2.4) is a linear combination of only these operators, the definition of Cartan prolongation itself ensures an isomorphism of $\left(\mathfrak{g}^{\lambda}\right)_{n}$ with $\left(\mathfrak{f}^{\lambda}\right)_{n}$.

2.2. The right inverse of $\Delta$ on $\mathfrak{s l e}^{\circ}$. Let $f$ be an arbitrary homogeneous with respect to the degree in $u$ and $\xi$ harmonic function, distinct from $\xi_{1} \ldots \xi_{n}$, i.e., an arbitrary generating function for $\mathfrak{s l e}^{\circ}(n)$. Then $f=\Delta(F)$ for some function $F$ (as follows from the computation of the homology of $\Delta$ which is an easy exersise; the answer: the homology space $H(\Delta)$ is spanned by $\left.\xi_{1} \ldots \xi_{n}\right)$. Clearly, $F$ is determined up to an arbitrary harmonic summand. Set $\Phi=\sum u_{i} \xi_{i}$. Then

$$
\Delta(\Phi f)=(\Delta \Phi) f-\Phi \Delta f-\{\Phi, f\}=\left(n+\operatorname{deg}_{u} f-\operatorname{deg}_{\xi} f\right) f .
$$

Define the right inverse of $\Delta$ by the formula

$$
\Delta^{-1} f=\frac{1}{\nu(f)}(\Phi f), \text { where } \nu(f)=n+\operatorname{deg}_{u} f-\operatorname{deg}_{\xi} f .
$$

Since the kernel of $\Delta$ is nonzero, $\Delta$ has no inverse. Still, $\Delta^{-1}$ maps $\mathfrak{s l e}^{\circ}(n)$ into $\mathfrak{l}(n)$ without kernel and on $\mathfrak{s} \mathfrak{e}^{\circ}(n)$ the following formula holds:

$$
\Delta\left(\Delta^{-1} f\right)=f \text { for } \operatorname{Le}_{f} \in \mathfrak{s l e}{ }^{\circ}(n) .
$$

2.3. Theorem. Any vector field $D \in \mathfrak{g}^{\lambda}$ can be represented in the form

$$
D=D_{f}+c Z \text {, where } c \in \mathbb{C} \text { and } Z=\sum_{i \leq 4}\left(u_{i} \frac{\partial}{\partial u_{i}}+\xi_{i} \frac{\partial}{\partial \xi_{i}}\right),
$$

where $\operatorname{Le}_{f} \in \mathfrak{s l e}^{\circ}(4)$ and where (recall that $A_{n} \subset S_{n}$ denotes the subgroup of even permutations):

$$
\begin{aligned}
& D_{f}=\operatorname{Le}_{f}+\lambda\left(-\operatorname{Le}_{\hat{f}}+2 \sum_{1 \leq i \leq 4 ;(i, j, k, l) \in A_{4}} \frac{\partial^{3}\left(\Delta^{-1}(f)\right)}{\partial \xi_{j} \partial \xi_{k} \partial \xi_{l}} \frac{\partial}{\partial \xi_{i}}\right) \\
& \text { for } \hat{f}=4 \Delta^{-1}\left(\frac{\partial^{4}\left(\Delta^{-1}(f)\right)}{\partial \xi_{1} \partial \xi_{2} \partial \xi_{3} \partial \xi_{4}}\right) .
\end{aligned}
$$

For the proof see Appendix 1.

Corollary. 1) The Lie superalgebra $\mathfrak{g}^{\lambda}$ is a deformation of $\mathfrak{s l} \mathfrak{e}^{\circ}(4) \boxplus \mathbb{C} \cdot Z$.

2) If $\operatorname{deg}_{\xi} f \leq 1$, then $D_{f}=\operatorname{Le}_{f}$, hence, $\mathfrak{h}=\left\{c \cdot Z+D_{f}: \operatorname{deg}_{\xi} f \leq 1, c \in \mathbb{C}\right\}$ remains rigid under this deformation.

3) Let $\Omega=d u_{1} \wedge d u_{2} \wedge d u_{3} \wedge d u_{4}$ be the volume element on the underlying manifold of the $\mathcal{C}^{4 \mid 4}$. Observe that the volume element $\operatorname{vol}(u, \xi)$ on the whole $\mathcal{C}^{4 \mid 4}$ is invariant with respect to the $\mathfrak{g}^{\lambda}$-action, but $\Omega$ is not invariant. It is invariant, however, with respect to the nondeformed subalgebra $\mathfrak{h}$. 
4) Let $D \in \mathfrak{g}^{\lambda}$; let $L_{D}$ be the Lie derivative. Denote by $\nabla=\sum \frac{\partial}{\partial u_{i}} \frac{\partial}{\partial \xi_{i}}$ the bivector dual to $\omega$. Observe that the left hand sides of equations (2.1)-(2.4) determine the coefficients of the 2-form $L_{D}(\omega)$ :

equations (2.1) determine the coefficients of $d u_{j} d \xi_{i}$;

equations (2.2) determine the coefficients of $d u_{i} d \xi_{i}$;

equations (2.3) determine the coefficients of $d \xi_{j} d \xi_{i}$;

equations (2.4) determine the coefficients of $d u_{j} d u_{i}$.

The right hand sides of (2.2) determine the nonzero coefficients of the form $\frac{1}{2}\left(\sum \frac{\partial Q_{i}}{\partial u_{i}}\right) \omega$, while the right hand sides of (2.4) determine the nonzero coefficients of the form $\lambda\left(L_{D} \Omega\right) * \nabla$, where $*$ is the convolution of tensors.

Therefore, (2.1)-(2.4) can be rewritten in the form

$$
L_{D} \omega=\frac{1}{2}\left(\sum \frac{\partial Q_{i}}{\partial u_{i}}\right) \omega+\lambda\left(L_{D} \Omega\right) * \nabla .
$$

If we replace coefficient of $\omega$ in the right hand side of (2.9) with an arbitrary function $\Psi(u, \xi)$ and add the constraint

$$
\operatorname{div}(D)=\sum\left(\frac{\partial Q_{i}}{\partial u_{i}}-(-1)^{p(D)} \frac{\partial P_{i}}{\partial \xi_{i}}\right)=0,
$$

then the sum of the four equations (2.2) with (2.10) automatically yields

$$
\Psi(u, \xi)=\frac{1}{2} \sum \frac{\partial Q_{i}}{\partial u_{i}} .
$$

Thus, we can distinguish the Lie superalgebra $\mathfrak{g}^{\lambda}$ by

$$
\left\{\begin{array}{ccc}
L_{D} \omega & = & \Psi \cdot \omega \\
\operatorname{div}(D) & = & 0
\end{array}\right.
$$

\section{The exceptional Lie subsuperalgebra kas of $\mathfrak{k}(1 \mid 6)$}

If the operator $d$ that determines a $\mathbb{Z}$-grading of the Lie superalgebra $\mathfrak{g}$ does not belong to $\mathfrak{g}$, we denote the Lie superalgebra $\mathfrak{g} \nexists \mathbb{C} \cdot d$ by $\mathfrak{d} \mathfrak{g}$. Recall also that $\mathfrak{c}(\mathfrak{g})$ or just $\mathfrak{c g}$ denotes the trivial 1-dimensional central extension of $\mathfrak{g}$ with the even center.

3.1. The Lie superalgebra $\mathfrak{g}=\mathfrak{k}(1 \mid 2 n)$ is generated by the functions from $\mathbb{C}[t, \xi, \eta]$, where $\xi=\left(\xi_{1}, \ldots, \xi_{n}\right), \eta=\left(\eta_{1}, \ldots, \eta_{n}\right)$. The standard $\mathbb{Z}$-grading of $\mathfrak{g}$ is induced by the $\mathbb{Z}$-grading of $\mathbb{C}[t, \xi, \eta]$ given by $\operatorname{deg} t=2, \operatorname{deg} \xi_{i}=\operatorname{deg} \eta_{i}=1$; namely, $\operatorname{deg} K_{f}=\operatorname{deg} f-2$. Clearly, in this grading $\mathfrak{g}$ is of depth 2. Let us consider the functions that generate several first homogeneous components of $\mathfrak{g}=\bigoplus_{i \geq-2} \mathfrak{g}_{i}$ :

\begin{tabular}{|c|c|c|c|c|}
\hline component & $\mathfrak{g}_{-2}$ & $\mathfrak{g}_{-1}$ & $\mathfrak{g}_{0}$ & $\mathfrak{g}_{1}$ \\
\hline its generators & 1 & $\Lambda^{1}(\xi, \eta)$ & $\Lambda^{2}(\xi, \eta) \oplus \mathbb{C} \cdot t$ & $\Lambda^{3}(\xi, \eta) \oplus t \Lambda^{1}(\xi, \eta)$ \\
\hline
\end{tabular}

As one can prove directly, the component $\mathfrak{g}_{1}$ generates the whole subalgebra $\mathfrak{g}_{+} \subset \mathfrak{g}$ of the elements of positive degree. The component $\mathfrak{g}_{1}$ splits into two $\mathfrak{g}_{0}$-modules $\mathfrak{g}_{11}=\Lambda^{3}$ and $\mathfrak{g}_{12}=t \Lambda^{1}$. It is obvious that $\mathfrak{g}_{12}$ is always irreducible and the component $\mathfrak{g}_{11}$ is trivial for $n=1$.

The Cartan prolongations of these components are well-known:

$$
\begin{gathered}
\left(\mathfrak{g}_{-} \oplus \mathfrak{g}_{0}, \mathfrak{g}_{11}\right)_{*}^{m k} \cong \mathfrak{p o}(0 \mid 2 n) \oplus \mathbb{C} \cdot K_{t} \cong \mathfrak{d}(\mathfrak{p o}(0 \mid 2 n)) \\
\left(\mathfrak{g}_{-} \oplus \mathfrak{g}_{0}, \mathfrak{g}_{12}\right)_{*}^{m k}=\mathfrak{g}_{-2} \oplus \mathfrak{g}_{-1} \oplus \mathfrak{g}_{0} \oplus \mathfrak{g}_{12} \oplus \mathbb{C} \cdot K_{t^{2}} \cong \mathfrak{o} \mathfrak{s p}(2 n \mid 2) .
\end{gathered}
$$


Observe a remarkable property of $\mathfrak{k}(1 \mid 6)$. For $n>1$ and $n \neq 3$ the component $\mathfrak{g}_{11}$ of $\mathfrak{k}(1 \mid 2 n)$ is irreducible. For $n=3$ the component splits into the two irreducible dual modules that we will denote by $\mathfrak{g}_{11}^{\xi}$ and $\mathfrak{g}_{11}^{\eta}$. Observe further, that $\mathfrak{g}_{0}=\mathfrak{o}(6) \cong \mathfrak{s l}(4)$. As $\mathfrak{s l}(4)$-modules, $\mathfrak{g}_{11}^{\xi}$ and $\mathfrak{g}_{11}^{\eta}$ are the symmetric squares $S^{2}(\mathrm{id})$ and $S^{2}\left(\mathrm{id}^{*}\right)$ of the identity 4-dimensional representation and its dual, respectively. Explicitly,

$$
\begin{array}{r}
\mathfrak{g}_{11}^{\xi}=\operatorname{Span}\left(\xi_{1} \xi_{2} \xi_{3}, \xi_{1}\left(\xi_{2} \eta_{2}+\xi_{3} \eta_{3}\right), \xi_{2}\left(\xi_{1} \eta_{1}+\xi_{3} \eta_{3}\right), \eta_{3}\left(\xi_{1} \eta_{1}-\xi_{2} \eta_{2}\right), \xi_{1} \eta_{2} \eta_{3},\right. \\
\left.\xi_{3}\left(\xi_{1} \eta_{1}+\xi_{2} \eta_{2}\right), \eta_{2}\left(\xi_{1} \eta_{1}-\xi_{3} \eta_{3}\right), \xi_{2} \eta_{1} \eta_{3}, \eta_{1}\left(\xi_{2} \eta_{2}-\xi_{3} \eta_{3}\right), \xi_{3} \eta_{1} \eta_{2}\right)
\end{array}
$$

and $\mathfrak{g}_{11}^{\eta}$ is obtained from $\mathfrak{g}_{11}^{\xi}$ after the replacement $\xi \longleftrightarrow \eta$.

3.2. Theorem. 1) The Cartan prolong $\left(\mathfrak{g}_{-} \oplus \mathfrak{g}_{0}, \mathfrak{g}_{11}^{\xi} \oplus \mathfrak{g}_{12}\right)_{*}^{m k}$ is infinite dimensional and simple. It is isomorphic to $\left(\mathfrak{g}_{-} \oplus \mathfrak{g}_{0}, \mathfrak{g}_{11}^{\eta} \oplus \mathfrak{g}_{12}\right)_{*}^{m k}$.

2) $\left(\mathfrak{g}_{-} \oplus \mathfrak{g}_{0}, \mathfrak{g}_{11}^{\xi}\right)_{*}^{m k} \cong\left(\mathfrak{g}_{-} \oplus \mathfrak{g}_{0}, \mathfrak{g}_{11}^{\eta}\right)_{*}^{m k} \cong \mathfrak{a} \mathfrak{s} \exists \mathbb{C} K_{t} \cong \mathfrak{d}(\mathfrak{a} \mathfrak{s})$.

Proof. Heading 2) is straightforward; the simplicity in heading 1) follows from Kac's criterion [K1]. To see that the Cartan prolong $\left(\mathfrak{g}_{-} \oplus \mathfrak{g}_{0}, \mathfrak{g}_{11}^{\xi} \oplus \mathfrak{g}_{12}\right)_{*}^{m k}$ is infinite dimensional we need the following lemma which clinches the proof.

Lemma. Denote $\mathfrak{h}=\left(\mathfrak{g}_{-} \oplus \mathfrak{g}_{0}, \mathfrak{g}_{11}^{\xi} \oplus \mathfrak{g}_{12}\right)_{*}^{m k}$. Consider the $\mathbb{Z}$-grading of $\mathfrak{h}$ induced by the standard grading of $\mathfrak{k}(1 \mid 6)$.

For $k>1$ the operator $T_{k}=\left.\left(\operatorname{ad} K_{t^{2}}\right)\right|_{\mathfrak{h}_{k}}$ determines an isomorphism of $\mathfrak{s l}(4)$ modules $\mathfrak{h}_{k}$ and $\mathfrak{h}_{k+2}$. The operator $T_{1}=\left.\left(\operatorname{ad} K_{t^{2}}\right)\right|_{\mathfrak{g}_{11}^{\xi}}$ determines an isomorphism of $\mathfrak{g}_{11}^{\xi}$ with its image.

Proof. We easily check that $K_{t^{2}} \in \mathfrak{h}$ and

$$
\text { ad } K_{t^{2}}=2 t(t \partial t+E-2), \quad \text { where } E=\sum\left(\xi_{i} \frac{\partial}{\partial \xi_{i}}+\eta_{i} \frac{\partial}{\partial \eta_{i}}\right) .
$$

Therefore, $\operatorname{Ker}\left(\operatorname{ad} K_{t^{2}}\right)$ in $\mathfrak{k}(1 \mid 6)$ consists of the fields generated by the functions $f$ such that $\operatorname{deg}_{t} f+\operatorname{deg}_{\xi} f+\operatorname{deg}_{\eta} f-2=0$, i.e., $\operatorname{Ker}\left(\operatorname{ad} K_{t^{2}}\right) \cong \mathfrak{s l}(4) \oplus \mathfrak{g}_{12} \oplus \mathbb{C} K_{t^{2}}$.

This makes it clear that, first, ad $K_{t^{2}}$ is $\mathfrak{s l}(4)$-invariant; second, the operators $T_{k}$ have no kernel for $k>0$.

We will denote the simple exceptional Lie superalgebra described in heading 1) of Theorem 3.2 by $\mathfrak{k} \mathfrak{a}$.

\section{Prolongs of the Lie superalgebras $\mathfrak{c g}$.}

The exceptional Cartan prolong $\mathfrak{v} \mathfrak{e}(4 \mid 3)=\left(\Pi\left(T_{0}(\overrightarrow{0})\right), \mathfrak{c v e c t}(0 \mid 3)\right)_{*}$

In order to construct this exceptional example we have to recall (see sec. 0.7) that on the supermanifold of purely odd dimension the space of volume forms is $T(\overrightarrow{-1})$ and the space of half-densities is $T(\overrightarrow{-1 / 2})$ (not $T(\overrightarrow{1})$ and $T(\overrightarrow{1 / 2})$ as on manifolds).

4.1. Let us now describe a construction of several exceptional simple Lie superalgebras. Let $\mathfrak{u}=\operatorname{vect}(m \mid n)$, let $\mathfrak{g}=\left(\mathfrak{u}_{-1}, \mathfrak{g}_{0}\right)_{*}$ be a simple Lie subsuperalgebra of $\mathfrak{u}$. Moreover, let there exist an element $D \in \mathfrak{u}_{0}$ that determines an exterior derivation of $\mathfrak{g}$ and has no kernel on $\mathfrak{u}_{+}=\bigoplus_{i>0} \mathfrak{u}_{i}$. Let us study the prolong $\tilde{\mathfrak{g}}=\left(\mathfrak{g}_{-1}, \mathfrak{g}_{0} \oplus \mathbb{C} D\right)_{*}$.

Lemma. Either $\tilde{\mathfrak{g}}$ is simple or $\tilde{\mathfrak{g}}=\mathfrak{g} \boxplus \mathbb{C} D$, where $\mathfrak{g}=\left(\mathfrak{u}_{-1}, \mathfrak{g}_{0}\right)_{*}$. 
Proof. Let $I$ be a nonzero graded ideal of $\tilde{\mathfrak{g}}$. The subsuperspace $\left(\operatorname{ad} \mathfrak{u}_{-1}\right)^{k+1} a$ of $\mathfrak{u}_{-1}$ is nonzero for any nonzero homogeneous element $a \in \mathfrak{u}_{k}$ and $k \geq 0$. Since $\mathfrak{g}_{-1}=\mathfrak{u}_{-1}$, the ideal $I$ contains nonzero elements from $\mathfrak{g}_{-1}$; by simplicity of $\mathfrak{g}$ the ideal $I$ contains the whole $\mathfrak{g}$. If, moreover, $\left[\mathfrak{g}_{-1}, \tilde{\mathfrak{g}}_{1}\right]=\mathfrak{g}_{0}$, then by definition of the Cartan prolongation $\tilde{\mathfrak{g}}=\mathfrak{g} \nexists \mathbb{C} D$.

If, instead, $\left[\mathfrak{g}_{-1}, \tilde{\mathfrak{g}}_{1}\right]=\mathfrak{g}_{0} \oplus \mathbb{C} D$, then $D \in I$ and since $\left[D, \mathfrak{u}_{+}\right]=\mathfrak{u}_{+}$, we derive that $I=\mathfrak{g}$. In other words, $\mathfrak{g}$ is simple.

4.2. Example 1. Take $\mathfrak{u}=\operatorname{vect}\left(2^{n-1} \mid 2^{n-1}-1\right)$. Consider $\mathfrak{u}_{-1}$ as $\Pi\left(T_{0}(\overrightarrow{0})\right)$ and set $\mathfrak{g}_{-1}=\mathfrak{u}_{-1}, \mathfrak{g}_{0}=\mathfrak{v e c t}(0 \mid n)$. Clearly, $\mathfrak{g}_{-1}$ is a $\mathfrak{g}_{0}$-module. Then $\left(\mathfrak{g}_{-1}, \mathfrak{g}_{0}\right)_{*}$ is a simple Lie superalgebra isomorphic to $\mathfrak{l}(n ; n)$. The isomorphism is established with the help of a regrading. For the operator $D$ of the exterior derivation of $\mathfrak{l}(n ; n)$ we take the grading operator $d \in \mathfrak{m}(n ; n)_{0} \subset \mathfrak{u}_{0}$, i.e., $\mathfrak{g}_{0} \oplus \mathbb{C} D \cong \mathfrak{c v e c t}(0 \mid n)$.

In particular, for $n=2$ we have $\mathfrak{g}_{-1}=\Pi\left(\xi_{1}, \xi_{2}, \xi_{1} \xi_{2}\right) ; \mathfrak{g}_{0}=\mathfrak{v e c t}(0 \mid 2) \cong \mathfrak{s l}(2 \mid 1)$. Then $\mathfrak{c}\left(\mathfrak{g}_{0}\right)=\mathfrak{g l}(2 \mid 1)$ and $\left(\Pi\left(T_{0}(\overrightarrow{0})\right), \mathfrak{c v e c t}(0 \mid 2)\right)_{*} \cong \mathfrak{v e c t}(2 \mid 1)$.

Theorem. 1) $\mathfrak{v} \mathfrak{e}(4 \mid 3)=\left(\Pi\left(T_{0}(\overrightarrow{0})\right) \text {, } \mathfrak{c} \mathfrak{v e c t}(0 \mid 3)\right)_{*}$ is a simple Lie superalgebra.

2) $\left(\Pi\left(T_{0}(\overrightarrow{0})\right), \mathfrak{c} \mathfrak{c} \mathfrak{e c t}(0 \mid n)\right)_{*} \cong \mathfrak{d}(\mathfrak{k} \mathfrak{e}(n ; n))$ for $n>3$.

Proof. Thanks to Lemma 4.1 heading 1) follows from the fact that the Cartan prolong $\left(\Pi\left(T_{0}(\overrightarrow{0})\right), \mathfrak{c v e c t}(0 \mid 3)\right)_{*}$ is bigger than $\mathfrak{s l e}^{\circ}(3 ; 3) \boxplus \mathbb{C} D$; we will prove this fact in $\S 5$. Heading 2 ) is proved in Appendix 2.

4.3. To clarify the structure of $\mathfrak{v l e}(4 \mid 3)=\left(\Pi\left(T_{0}(\overrightarrow{0})\right), \mathfrak{c} \mathfrak{v e c t}(0 \mid 3)\right)_{*}$, consider one more construction. Let us describe one wonderful property of $\mathfrak{s l e}{ }^{\circ}(3)$ that singles it out among the $\mathfrak{s l e} \mathfrak{e}^{\circ}(n)$.

In the standard grading of $\mathfrak{g}=\mathfrak{s} \mathfrak{e}^{\circ}(3)$ we have $\operatorname{dim} \mathfrak{g}_{-1}=(3,3), \mathfrak{g}_{0} \cong \mathfrak{s p \mathfrak { e }}(3)$. For the regraded superalgebra $R \mathfrak{g}=\mathfrak{s l e}{ }^{\circ}(3 ; 3)$ we have $\operatorname{dim} R \mathfrak{g}_{-1}=(3,3), R \mathfrak{g}_{0}=$ $\mathfrak{s v e c t}(0 \mid 3) \cong \mathfrak{s p e}(3)$. Therefore, for $\mathfrak{s l e}^{\circ}(3)$ and only for it among the $\mathfrak{s l e}^{\circ}(n)$, the regrading $R$ determines a nontrivial automorphism. In terms of generating functions the regrading $R$ is given by the formulas:

1) if $\operatorname{deg}_{\xi}(f)=0$, then $R(f)=\Delta\left(f \xi_{1} \xi_{2} \xi_{3}\right)$;

2) if $\operatorname{deg}_{\xi}(f)=1$, then $R(f)=f$

3) if $\operatorname{deg}_{\xi}(f)=2$, then $R(f)=\frac{\partial^{3}\left(\Delta^{-1} f\right)}{\partial \xi_{1} \partial \xi_{2} \partial \xi_{3}}($ see $(2.6))$.

We see that $R^{2}=S I G N$, where the operator $S I G N$ is defined by the formulas

$$
S I G N(D)=(-1)^{p(D)} D \text { on the vector fields }
$$

and

$$
S I G N(f)=(-1)^{p(f)+1} f \text { on the generating functions. }
$$

Let now $\mathfrak{g}=\mathfrak{l e}(3 ; 3)$ and $i_{1}: \mathfrak{g} \longrightarrow \mathfrak{u}=\mathfrak{v e c t}(4 \mid 3)$ be the embedding that preserves the standard $\mathbb{Z}$-grading of $\mathfrak{g}$. Let $\mathfrak{h}=\mathfrak{l}(3)$ and $\tilde{\mathfrak{h}}=\mathfrak{s} \mathfrak{e} \mathfrak{e}^{\circ}(3) \subset \mathfrak{h}$. Then the map

$$
i_{2}=S I G N \circ i_{1} R: \tilde{\mathfrak{h}} \longrightarrow \mathfrak{u}
$$

is an embedding that preserves the grading of $\tilde{\mathfrak{h}}$.

Observe that $\tilde{\mathfrak{h}}_{0}=\mathfrak{s p e}(3)$ and $\mathfrak{h}_{0}=\mathfrak{p e}(3) \cong \tilde{\mathfrak{h}}_{0} \oplus \mathbb{C}\left(\sum q_{i} \xi_{i}\right)$. On the space $i_{2}\left(\mathfrak{h}_{-1}\right)$, the action of $z=-2 i_{1}\left(\sum q_{i} \xi_{i}\right)+3 d$ coincides with the action of $\sum q_{i} \xi_{i}$ on $\mathfrak{h}_{-1}$. Therefore, setting $i_{2}\left(\sum q_{i} \xi_{i}\right)=z$ we get an embedding $i_{2}\left(\mathfrak{h}_{-1} \oplus \mathfrak{h}_{0}\right) \longrightarrow \mathfrak{u}$ that can be extended to an embedding of $\mathfrak{h}$ to $\mathfrak{u}$. Under this embedding

$$
i_{1}\left(\mathfrak{g}_{-1} \oplus \mathfrak{g}_{0}\right)+i_{2}\left(\mathfrak{h}_{-1} \oplus \mathfrak{h}_{0}\right)=\mathfrak{u}_{-1} \oplus\left(\mathfrak{g}_{0} \oplus \mathbb{C} d\right),
$$


i.e., the nondirect sum of the images of $i_{1}$ and $i_{2}$ covers the whole nonpositive part of $\mathfrak{v} \mathfrak{e}(4 \mid 3)$.

Thus, there are two distinct embeddings of $\mathfrak{l}(3) \cong \mathfrak{l}(3 ; 3)$ into $\left(\Pi\left(T_{0}(\overrightarrow{0})\right)\right.$, $\mathfrak{c} \mathfrak{e} e \mathfrak{c t}(0 \mid 3))_{*}$ :

$$
i_{1}: \mathfrak{l e}(3 ; 3) \longrightarrow \mathfrak{v l e}(4 \mid 3)
$$

and

$$
i_{2}: \mathfrak{l e}(3) \longrightarrow \mathfrak{v} \mathfrak{l e}(4 \mid 3)
$$

such that

$$
i_{1}(\mathfrak{l} \mathfrak{e}(3 ; 3))+i_{2}(\mathfrak{l} \mathfrak{e}(3))=\mathfrak{v} \mathfrak{k}(4 \mid 3)
$$

(the sum in the left hand side is not a direct one!). As a linear space, $\mathfrak{v} \mathfrak{e}(4 \mid 3)$ is the quotient of $\mathfrak{l e}(3 ; 3)) \oplus \mathfrak{l e}(3)$ modulo the subspace $V=\{(S I G N \circ R g \oplus-g): g \in$ $\left.\mathfrak{s l e}^{\circ}(3)\right\}$. The map $\varphi$ defined by the formula

$$
\left.\varphi\right|_{i_{1}(\mathfrak{l e}(3 ; 3))}=S I G N \circ i_{2} i_{1}^{-1} ;\left.\quad \varphi\right|_{i_{2}(\mathfrak{l e}(3))}=i_{1} i_{2}^{-1}
$$

determines a nontrivial automorphism of $\mathfrak{v} \mathfrak{k}(4 \mid 3)$.

\section{AN EXPLICIT FORM OF THE VECTOR FIELDS FROM $\mathfrak{v} \mathfrak{e}(4 \mid 3)=\left(\Pi\left(T_{0}(\overrightarrow{0})\right), \mathfrak{c v e c t}(0 \mid 3)\right)_{*} \subset \mathfrak{v} \mathfrak{v e c t}(4 \mid 3)$}

Here we consider $\mathfrak{v} \mathfrak{k}(4 \mid 3)_{0}=\mathfrak{c} \mathfrak{v e c t}(0 \mid 3)$ and the module $\mathfrak{v} \mathfrak{e}(4 \mid 3)_{-1}=\Pi\left(T_{0}(\overrightarrow{0})\right)$ over it. Let us identify this module with the quotient of $\Pi\left(\Lambda\left(\eta_{1}, \eta_{2}, \eta_{3}\right)\right)$ modulo constants and redenote the basis in $\Pi\left(\Lambda\left(\eta_{1}, \eta_{2}, \eta_{3}\right)\right)$ so that this quotient becomes spanned by the partial derivatives with respect to the certain new indeterminates. Namely, we set

$$
\Pi\left(\eta_{1} \eta_{2} \eta_{3}\right) \mapsto-\frac{\partial}{\partial y} ; \quad \Pi\left(\eta_{i}\right) \mapsto-\frac{\partial}{\partial u_{i}} ; \quad \Pi\left(\frac{\partial \eta_{1} \eta_{2} \eta_{3}}{\partial \eta_{i}}\right) \mapsto-\frac{\partial}{\partial \xi_{i}} .
$$

Every element $D \in \mathfrak{v e c t}(4 \mid 3)$ is of the form $D=\sum_{i \leq 3}\left(P_{i} \frac{\partial}{\partial \xi_{i}}+Q_{i} \frac{\partial}{\partial u_{i}}\right)+R \frac{\partial}{\partial y}$, where $P_{i}, Q_{i}, R \in \mathbb{C}[y, u, \xi]$.

5.1. Lemma. Set $\mathfrak{g}_{-1}=\operatorname{Span}\left(\frac{\partial}{\partial y} ; \frac{\partial}{\partial u_{i}}, \frac{\partial}{\partial \xi_{i}}\right.$ for $\left.i \leq 3\right), \mathfrak{g}_{0}=\mathfrak{c v e c t}(0 \mid 3)$. The homogeneous (with respect to parity) vector field $D \in \mathfrak{v e c t}(4 \mid 3)$ belongs to $\left(\mathfrak{g}_{-1}, \mathfrak{g}_{0}\right)_{*}$ if and only if it satisfies the following system of equations:

$$
\begin{gathered}
\frac{\partial Q_{i}}{\partial u_{j}}+(-1)^{p(D)} \frac{\partial P_{j}}{\partial \xi_{i}}=0 \text { for any } i \neq j ; \\
\frac{\partial Q_{i}}{\partial u_{i}}+(-1)^{p(D)} \frac{\partial P_{i}}{\partial \xi_{i}}=\frac{1}{2}\left(\sum_{1 \leq j \leq 3} \frac{\partial Q_{j}}{\partial u_{j}}+\frac{\partial R}{\partial y}\right) \text { for } i=1,2,3 ; \\
\frac{\partial Q_{i}}{\partial \xi_{j}}+\frac{\partial Q_{j}}{\partial \xi_{i}}=0 \text { for any } i, j ; \text { in particular } \frac{\partial Q_{i}}{\partial \xi_{i}}=0 ; \\
\frac{\partial P_{i}}{\partial u_{j}}-\frac{\partial P_{j}}{\partial u_{i}}=-(-1)^{p(D)} \frac{\partial R}{\partial \xi_{k}} \text { for any even permutation }\left(\begin{array}{ccc}
1 & 2 & 3 \\
i & j & k
\end{array}\right) ; \\
\frac{\partial Q_{i}}{\partial y}=0 \text { for } i=1,2,3 ;
\end{gathered}
$$




$$
\frac{\partial P_{k}}{\partial y}=(-1)^{p(D)} \frac{1}{2}\left(\frac{\partial Q_{i}}{\partial \xi_{j}}-\frac{\partial Q_{j}}{\partial \xi_{i}}\right) \text { for any even permutation }\left(\begin{array}{ccc}
1 & 2 & 3 \\
i & j & k
\end{array}\right) .
$$

Proof is similar to that of Lemma 2.1.

Remark. The left hand sides of (5.1)-(5.6) determine the coefficients of the 2 -form $L_{D} \omega$, where $L_{D}$ is the Lie derivative and $\omega=\sum_{1 \leq i \leq 3} d u_{i} d \xi_{i}$. It is interesting to interpret the right hand sides of these equations.

5.2. Theorem. Every solution of the system (5.1)-(5.6) is of the form:

$$
\begin{aligned}
D= & \operatorname{Le}_{f}+y B_{f}-(-1)^{p(f)}\left(y \Delta(f)+y^{2} \frac{\partial^{3} f}{\partial \xi_{1} \partial \xi_{2} \partial \xi_{3}}\right) \frac{\partial}{\partial y} \\
& +B_{g}-(-1)^{p(g)}\left(\Delta(g)+2 y \frac{\partial^{3} g}{\partial \xi_{1} \partial \xi_{2} \partial \xi_{3}}\right) \frac{\partial}{\partial y}
\end{aligned}
$$

where $f, g \in \mathbb{C}[u, \xi]$ are arbitrary and the operator $B_{f}$ is given by the formula:

$$
B_{f}=\frac{\partial^{2} f}{\partial \xi_{2} \partial \xi_{3}} \frac{\partial}{\partial \xi_{1}}+\frac{\partial^{2} f}{\partial \xi_{3} \partial \xi_{1}} \frac{\partial}{\partial \xi_{2}}+\frac{\partial^{2} f}{\partial \xi_{1} \partial \xi_{2}} \frac{\partial}{\partial \xi_{3}} .
$$

Proof is similar to that of Theorem 2.3; see Appendix 1 and [ShP].

Formula (5.7) makes it possible to explicitly express the two embeddings (4.1.1)

$$
i_{1}, i_{2}: \mathfrak{l} \mathfrak{e}(3) \longrightarrow \mathfrak{v} \mathfrak{k}(4 \mid 3) \text {. }
$$

The first embedding $i_{1}$ preserves the grading of $\mathfrak{k}(3 ; 3)$, cf. 0.4 . I do not know any compact general formula for $i_{1}$ and can only determine it component-wise (mind that $A_{3}$ in the first line of the following displayed formula is the group of even permutations):

$$
\begin{gathered}
i_{1}\left(\operatorname{Le}_{f(u)}\right)=\operatorname{Le}_{\sum \frac{\partial f}{\partial u_{i}} \xi_{j} \xi_{k}-y f} \text {, where } y \text { is treated as a parameter } \\
\text { and }(i, j, k) \in A_{3} ; \\
i_{1}\left(\operatorname{Le}_{\left.\sum f_{i}(u) \xi_{i}\right)=} \operatorname{Le}_{f}-\varphi(u) \sum \xi_{i} \frac{\partial}{\partial \xi_{i}}+\left(-\varphi(u) y+\Delta\left(\varphi(u) \xi_{1} \xi_{2} \xi_{3}\right)\right) \frac{\partial}{\partial y},\right. \\
\text { where } \varphi(u)=\Delta(f) ; \\
i_{1}\left(\operatorname{Le}_{1 \leq i \leq 3 ;(i, j, k) \in A_{3}} \psi_{i}(u) \xi_{k} \xi_{l}\right)=B_{f}-\Delta(f) \frac{\partial}{\partial y}, \text { where } B_{f} \text { is given by }(5.8) ; \\
i_{1}\left(\operatorname{Le}_{\psi(u) \xi_{1} \xi_{2} \xi_{3}}\right)=-\psi(u) \frac{\partial}{\partial y} .
\end{gathered}
$$

The second embedding $i_{2}$ preserves the standard grading of $\mathfrak{l}(3)$. It is given by the formulas

$$
i_{2}\left(\operatorname{Le}_{f}\right)=\operatorname{Le}_{f}+y B_{f}-(-1)^{p(f)}\left(y \Delta(f)+y^{2} \frac{\partial^{3} f}{\partial \xi_{1} \partial \xi_{2} \partial \xi_{3}}\right) \frac{\partial}{\partial y} .
$$

6. The eXCePtional Simple Lie Superalgebras of DePth 2:

$$
\mathfrak{m} \mathfrak{b}(4 \mid 5)=(\mathfrak{a} \mathfrak{b}(4), \mathfrak{c v e c t}(0 \mid 3))_{*}^{m} \text { AND } \mathfrak{k} \mathfrak{s} \mathfrak{e}(9 \mid 6)=\left(\mathfrak{h e i}(8 \mid 6), \mathfrak{s v e c t}(4)_{3,4}\right)_{*}^{k}
$$

Two more examples of exceptional simple Lie superalgebras are obtained with the help of a construction that generalizes the constructions from $\S 4$ to Lie superalgebras of depth 2. Let $\mathfrak{u}=\bigoplus_{i \geq-2} \mathfrak{u}_{i}$ be either $\mathfrak{m}(n)$ or $\mathfrak{k}(2 m+1 \mid n) ;$ let $\mathfrak{g}=\left(\mathfrak{u}_{-}, \mathfrak{g}_{0}\right)_{*}$ 
be a subalgebra of $\mathfrak{u}$ such that the subspace $\mathfrak{u}_{-2}$ belongs to the center of $\mathfrak{g}$ and the quotient $\mathfrak{g} / \mathfrak{u}_{-2}$ is simple. Moreover, let $D \in \mathfrak{u}_{0}$ determine an exterior derivation of $\mathfrak{g}$ without kernel on $\mathfrak{u}_{-2} \oplus \mathfrak{u}_{+}$, where $\mathfrak{u}_{+}=\bigoplus_{i>0} \mathfrak{u}_{i}$.

Let us study the $m k$-prolong $\tilde{\mathfrak{g}}=\left(\mathfrak{g}_{-}, \mathfrak{g}_{0} \oplus \mathbb{C} D\right)_{*}^{m k}$. The main result of this section: a description of two simple exceptional Lie superalgebras $\mathfrak{m} \mathfrak{b}(4 \mid 5)=(\mathfrak{a} \mathfrak{b}(4)$, $\mathfrak{c b e c t}(0 \mid 3))_{*}^{m}\left(\right.$ Theorem 6.2) and $\mathfrak{k} \mathfrak{s} \mathfrak{e}(9 \mid 6)=\left(\mathfrak{h e i}(8 \mid 6), \mathfrak{s v e c t}_{3,4}(4)\right)_{*}^{k}($ Theorem 6.5).

6.1. Lemma. Either $\tilde{\mathfrak{g}}$ is simple or $\tilde{\mathfrak{g}} \cong \mathfrak{g} \nexists \mathbb{C} D$, where $\mathfrak{g}=\left(\mathfrak{u}_{-}, \mathfrak{g}_{0}\right)_{*}$.

Proof. First, let us prove that if $\tilde{\mathfrak{g}} ¥ \mathfrak{g} \nexists \mathbb{C} D$, then $\mathfrak{u}_{-2}$ is not an ideal in $\tilde{\mathfrak{g}}$. Indeed, in this case there exist $g_{-1} \in \mathfrak{g}_{-1}, g_{0} \in \mathfrak{g}_{0}$ and $g_{1} \in \tilde{\mathfrak{g}}_{1}$ such that $\left[g_{1}, g_{-1}\right]=D+g_{0}$.

Let $\mathfrak{u}_{-2}=\mathbb{C} z$. Then $\left[g_{-1}, z\right]=\left[g_{0}, z\right]=0$ and we have

$$
\begin{aligned}
& {\left[g_{-1},\left[z, g_{1}\right]\right]=\left[\left[g_{-1}, z\right], g_{1}\right]+(-1)^{p\left(g_{-1}\right) p(z)}\left[z,\left[g_{-1}, g_{1}\right]\right]} \\
& +(-1)^{p\left(g_{-1}\right) p(z)}\left[z, D+g_{0}\right]=(-1)^{p\left(g_{-1}\right) p(z)}[z, D] \neq 0 .
\end{aligned}
$$

(We have taken into account that $D$ has no kernel on $\mathfrak{u}_{-2}$.) Hence, $\left[z, g_{1}\right]$ is a nonzero element of $\mathfrak{g}_{-1}$. The rest of the proof mimics that of Lemma 4.1

6.2. Consider $\hat{\mathfrak{g}}=\mathfrak{b}_{1 / 2}(n ; n)$. We have

$$
\hat{\mathfrak{g}}_{-1}=\Pi(T(\overrightarrow{-1 / 2})) ; \quad \hat{\mathfrak{g}}_{0}=\mathfrak{c v e c t}(0 \mid n)
$$

and the $\hat{\mathfrak{g}}_{0}$-action on $\hat{\mathfrak{g}}_{-1}$ preserves the nondegenerate superskew-symmetric form

$$
B(\varphi \sqrt{v o l}, \psi \sqrt{v o l})=\int \varphi \psi \cdot v o l ; \quad p(B) \equiv n \quad(\bmod 2) .
$$

Now, let $\mathfrak{g}=\tilde{\mathfrak{c}}\left(\mathfrak{b}_{1 / 2}(3 ; 3)\right)$ be the corresponding to (6.1) nontrivial central extension (we indicate this fact by tilde over $\mathfrak{c}$ ) of $\hat{\mathfrak{g}}$. The depth of $\tilde{\mathfrak{c}}\left(\mathfrak{b}_{1 / 2}(3 ; 3)\right)$ is equal to 2 . This central extension is naturally embedded into

$$
\mathfrak{u}=\left\{\begin{array}{cc}
\mathfrak{m}\left(2^{n-1}\right) & \text { for } n \text { odd, } \\
\mathfrak{k}\left(1+2^{n-1} \mid 2^{n-1}\right) & \text { for } n \text { even. }
\end{array}\right.
$$

As the operator $D$ described in sec. 6.1 we take the grading operator $d \in \mathfrak{u}_{0}$, i.e., $\mathfrak{g}_{0} \oplus \mathbb{C} D \cong \mathfrak{c}\left(\mathfrak{g}_{0}\right)$.

Example. Let $n=2$. Then $\mathfrak{g}_{-1}=\Pi(T(\overrightarrow{-1 / 2}))$ and $\mathfrak{g}_{-}=\mathfrak{h} \mathfrak{i}(2 \mid 2)$. We also have $\mathfrak{c}\left(\mathfrak{g}_{0}\right)=\mathfrak{c b e c t}(0 \mid 2) \cong \mathfrak{c o s p}(2 \mid 2)$ and $(\mathfrak{h e i}(2 \mid 2), \mathfrak{c v e c t}(0 \mid 2))_{*}^{k} \cong \mathfrak{k}(3 \mid 2) ;$ see sec. 0.6.

Theorem. 1) $(\mathfrak{a} \mathfrak{b}(4), \mathfrak{c} \mathfrak{v e c t}(0 \mid 3))_{*}^{m}$ is a simple Lie superalgebra.

2) $\left(\mathfrak{a} \mathfrak{b}\left(2^{n-1}\right), \mathfrak{c} \mathfrak{v e c t}(0 \mid n)\right)_{*}^{m k} \cong \mathfrak{d}\left(\left(\mathfrak{a} \mathfrak{b}\left(2^{n-1}\right), \mathfrak{v e c t}(0 \mid n)\right)_{*}^{m k}\right) \cong \mathfrak{d}(\mathfrak{b}(n ; n))$ for $n>3$.

Proof. Thanks to Lemma 6.1 heading 1) follows from the fact that the $m$-prolongation of $(\mathfrak{a} \mathfrak{b}(4), \mathfrak{c} \mathfrak{e} \mathfrak{e c t}(0 \mid 3))$ is bigger than $(\mathfrak{a} \mathfrak{b}(4), \mathfrak{v e c t}(0 \mid 3))_{*}^{m}$. We give explicit formulas in Appendix 3. Heading 2) is proved in Appendix 2.

6.3. The exceptional extension $\mathfrak{e s t e}^{\circ}(3)$. Let us clarify the structure of the exceptional Lie superalgebra $(\mathfrak{a b}(4), \mathfrak{c v e c t}(0 \mid 3))_{*}^{m k}$ with the help of a construction similar to that from $\S 4$. To this end, we describe another remarkable property of $\mathfrak{s l e}^{\circ}(3)$ that singles it out among the $\mathfrak{s l e}^{\circ}(n)$.

The Lie superalgebra $\mathfrak{g}=\mathfrak{s l e}^{\circ}(3)$ has a $2 \varepsilon$-dimensional nontrivial central extension $\mathfrak{e s l} \mathfrak{e}^{\circ}(3)$ : the element $M_{1}$ of degree -2 with respect to the standard grading of $\mathfrak{s l} \mathfrak{e}^{\circ}(3)$ extends $\mathfrak{s l e}^{\circ}(3)$ to $\mathfrak{s b}^{\circ}(3)$ while $z$ of degree -2 with respect to the grading of $\mathfrak{s l e}^{\circ}(3 ; 3)$ is associated with the form $B$ on the space $\mathfrak{g}_{-1}$ of half-densities with shifted parity (see (6.1)) in the realization $\mathfrak{g}=\mathfrak{s l} \mathfrak{e}^{\circ}(3 ; 3)$. 
The regrading $R$ interchanges these central elements and establishes a nontrivial automorphism of $\mathfrak{e} \mathfrak{s l e}^{\circ}(3)$.

Now let $\mathfrak{g}=\tilde{\mathfrak{c}}\left(\mathfrak{b}_{1 / 2}(3 ; 3)\right)$ be the described in sec. 6.2 nontrivial central extension of depth 2 of $\mathfrak{b}_{1 / 2}(3 ; 3)$; clearly, $\mathfrak{g}_{-2}=\mathbb{C} z$. The inverse regrading $R^{-1}$ sends $\mathfrak{g}$ into the nontrivial central extension $\mathfrak{h}=\tilde{\mathfrak{c}}\left(\mathfrak{b}_{-3,1}(3)\right.$ ) of $\mathfrak{b}_{-3,1}(3)$ (see sec. 0.7) and $\operatorname{deg} R^{-1} z=-1$.

From the very beginning we have an embedding $i_{1}: \mathfrak{g} \longrightarrow \mathfrak{u}=\mathfrak{m}(4)$. Let $\tilde{\mathfrak{h}}=\mathfrak{e} \mathfrak{s} \mathfrak{e}^{\circ}(3) \subset \mathfrak{h}$.

Then the map $i_{2}=S I G N \circ i_{1} R$ determines an embedding of $\tilde{\mathfrak{h}}$ to $\mathfrak{u}$ preserving the grading of $\tilde{\mathfrak{h}}$.

Observe that $\tilde{\mathfrak{h}}_{0}=\mathfrak{s p e}(3)$ and $\mathfrak{h}_{0}=\mathfrak{p e}(3) \cong \tilde{\mathfrak{h}}_{0} \oplus \mathbb{C} \cdot M_{\sum q_{i} \xi_{i}-3 \tau}$. On $\mathfrak{h}_{-1}$, $z=M_{\sum q_{i} \xi_{i}-3 \tau}$ acts by the formula:

$$
z: M_{q} \mapsto 4 M_{q}, \quad M_{\xi} \mapsto 2 M_{\xi} .
$$

This action of $z$ coincides with the action of $-3 d+\frac{1}{2} i_{1}(z)$.

Therefore, the embedding $i_{2}$ of $\mathfrak{h}_{-}$can be extended to an embedding of the whole $\mathfrak{h}$. We have

$$
i_{1}\left(\mathfrak{g}_{-} \oplus \mathfrak{g}_{0}\right)+i_{2}\left(\mathfrak{h}_{-} \oplus \mathfrak{h}_{0}\right)=\mathfrak{u}_{-} \oplus\left(\mathfrak{g}_{0} \oplus \mathbb{C} d\right)
$$

i.e., the nondirect sum of the images of $i_{1}$ and $i_{2}$ covers the whole nonpositive part of $(\mathfrak{a} \mathfrak{b}(4), \mathfrak{c o v e c t}(0 \mid 3))_{*}^{m k}$.

Thus, we have two distinct embeddings of $\tilde{\mathfrak{c}}\left(\mathfrak{b}_{-3,1}(3)\right)$, isomorphic to $\tilde{\mathfrak{c}}\left(\mathfrak{b}_{1 / 2}(3 ; 3)\right)$ as abstract, but not as graded, Lie superalgebras, into $(\mathfrak{a b}(4), \mathfrak{c b e c t}(0 \mid 3))_{*}^{m k}$ :

$$
\begin{aligned}
i_{1}: \tilde{\mathfrak{c}}\left(\mathfrak{b}_{1 / 2}(3 ; 3)\right) \longrightarrow & (\mathfrak{a b}(4), \mathfrak{c} \mathfrak{c} \mathfrak{e c t}(0 \mid 3))_{*}^{m k} \\
& \text { with the grading of } \tilde{\mathfrak{c}}\left(\mathfrak{b}_{1 / 2}(3 ; 3)\right) \text { preserved }
\end{aligned}
$$

and

$$
\begin{aligned}
i_{2}: \tilde{\mathfrak{c}}\left(\mathfrak{b}_{-3,1}(3)\right) \longrightarrow & (\mathfrak{a} \mathfrak{b}(4), \mathfrak{c} \mathfrak{c} \mathfrak{e c t}(0 \mid 3))_{*}^{m k} \\
& \text { with the grading of } \tilde{\mathfrak{c}}\left(\mathfrak{b}_{-3,1}(3)\right) \text { preserved }
\end{aligned}
$$

such that

$$
i_{1}\left(\tilde{\mathfrak{c}}\left(\mathfrak{b}_{1 / 2}(3 ; 3)\right)\right)+i_{2}\left(\tilde{\mathfrak{c}}\left(\mathfrak{b}_{-3,1}(3)\right)\right)=(\mathfrak{a} \mathfrak{b}(4), \mathfrak{c} \mathfrak{v e c t}(0 \mid 3))_{*}^{m k}
$$

(the sum here is not a direct one!). As a linear space, $(\mathfrak{c b e c t}(0 \mid 3))_{*}^{m k}$ is the quotient of $\tilde{\mathfrak{c}}\left(\mathfrak{b}_{1 / 2}(3 ; 3)\right) \oplus \tilde{\mathfrak{c}}\left(\mathfrak{b}_{-3,1}(3)\right)$ modulo the subspace $V=\{(S I G N \circ R g \oplus-g): g \in$ $\left.\mathfrak{e} \mathfrak{s l e}{ }^{\circ}(3)\right\}$. The map $\varphi$ defined by the formulas

$$
\left.\varphi\right|_{i_{1}\left(\tilde{\mathfrak{c}}\left(\mathfrak{b}_{1 / 2}(3 ; 3)\right)\right)}=S I G N \circ i_{2} i_{1}^{-1} ;\left.\quad \varphi\right|_{i_{2}\left(\tilde{\mathfrak{c}}\left(\mathfrak{b}_{-3,1}(3)\right)\right)}=i_{1} i_{2}^{-1}
$$

determines a nontrivial automorphism of $(\mathfrak{a} \mathfrak{b}(4), \mathfrak{c} \mathfrak{e} \mathfrak{e c t}(0 \mid 3))_{*}^{m k}$.

6.4. Description of $\left(\mathfrak{h e i}(8 \mid 6), \mathfrak{s v e c t}(0 \mid 4)_{3,4}\right)_{*}^{m k}$. Consider the nontrivial central extension $\mathfrak{g}=\tilde{\mathfrak{c}}\left(\mathfrak{s l e}{ }^{\circ}(n ; n)\right)$ of $\mathfrak{s l e}{ }^{\circ}(n ; n)$ defined as follows. We have: $\mathfrak{g}_{0}=\mathfrak{s v e c t}(0 \mid n)$; $\mathfrak{g}_{-1}=\Pi\left(T^{0}(\overrightarrow{0}) / \mathbb{C} \cdot 1\right)$, where $T^{0}(\overrightarrow{0})=\left\{f \in T(\overrightarrow{0}): \int f v o l(\xi)=0\right\}$. Define the central extension with the help of the form $\omega$ on $\mathfrak{g}_{-1}$ given by the formula:

$$
\omega(f, g)=\int f g \cdot \operatorname{vol}(\xi) .
$$

The same arguments as in 6.2 , show that $\left(\mathfrak{g}_{-1}, \mathfrak{g}_{0}\right)_{*}^{m k}$ can be embedded into $\mathfrak{k}\left(1+2^{n-1} \mid 2^{n-1}-2\right)$ for $n$ even and into $\mathfrak{m}\left(2^{n-1}-1\right)$ for $n$ odd. 
Let $x$ be the operator determining the standard $\mathbb{Z}$-grading of $\mathfrak{s v e c t}(0 \mid n)$ and let $z$ commute with $\mathfrak{s v e c t}(0 \mid n)$; let $a, b \in \mathbb{C}$. For any $a, b$ the element $a x+b z$ determines an outer derivation of $\mathfrak{g}_{0}$. Set

$$
\mathfrak{s v e c t}_{a, b}(n)=\mathfrak{s v e c t}(0 \mid n) \boxplus \mathbb{C}(a x+b z) ;
$$

set also

$$
\begin{aligned}
&\left(\mathfrak{g}_{-}, \mathfrak{s} \mathfrak{v e c t} \mathfrak{c t}_{a, b}(n)\right)_{*}^{m k}=\left(\mathfrak{g}_{-}, \mathfrak{g}_{0}\right.\boxplus \mathbb{C}(a x+b z))_{*}^{m k}, \\
& \text { where } \mathfrak{g}_{-}= \begin{cases}\mathfrak{a} \mathfrak{b}\left(2^{n-1}-1\right) & \text { for } n \text { odd, } \\
\mathfrak{h} \mathfrak{e}\left(2^{n-1} \mid 2^{n-1}-2\right) & \text { for } n \text { even. }\end{cases}
\end{aligned}
$$

Example. Let $n=3$. Then $\mathfrak{g}_{-1}=\Pi\left(\xi_{1}, \xi_{2}, \xi_{3}, \xi_{1} \xi_{2}, \xi_{1} \xi_{3}, \xi_{2} \xi_{3}\right)$ and $\mathfrak{g}_{-} \cong$ $\left(\mathfrak{b}_{\lambda}(3)\right)_{-}=\mathfrak{a b}(3)$ for any $a, b$, while $\mathfrak{g}_{0}=\mathfrak{s v e c t}(0 \mid 3) \cong \mathfrak{s p e}(3)$. The operator $x$ becomes $\sum \xi_{i} \partial \xi_{i}$ and

$$
\mathfrak{g}_{0} \boxplus \mathbb{C}(a x+b z) \cong \mathfrak{s p e}(3) \boxplus \mathbb{C}\left(a \sum \xi_{i} \partial \xi_{i}+b z\right) \cong\left(\mathfrak{b}_{\lambda}(3)\right)_{0} \text { for } \lambda=-\frac{b}{3 a}
$$

Therefore, $\left(\mathfrak{a} \mathfrak{b}(3), \mathfrak{s v e c t}_{a, b}(3)\right)_{*}^{m k} \cong \mathfrak{b}_{\lambda}(3)$ for $\lambda=-\frac{b}{3 a}$. In particular,

$$
\left(\mathfrak{a b}(3), \mathfrak{s v e c t}_{1,3}(3)\right)_{*}^{m k} \cong \mathfrak{s m}(3) \text { and }\left(\mathfrak{a b}(3), \mathfrak{s v e c t}_{1,0}(3)\right)_{*}^{m k} \cong \mathfrak{b}(3) \text {. }
$$

6.5. Theorem. 1) $\left(\mathfrak{h e i}(8 \mid 6), \mathfrak{s v e c t}_{3,4}(4)\right)_{*}^{m k}$ is a simple Lie superalgebra.

$$
\begin{aligned}
& \text { 2) Let } \mathfrak{g}_{-}= \begin{cases}\mathfrak{a} \mathfrak{b}\left(2^{n-1}-1\right) & \text { for } n \text { odd } \\
\text { Then } & \text { for } n \text { even. } \\
\mathfrak{h e i}\left(2^{n-1} \mid 2^{n-1}-2\right)\end{cases} \\
& \left(\mathfrak{g}_{-}, \mathfrak{s v e c t}_{a, b}(n)\right)_{*}^{m k} \cong\left(\mathfrak{g}_{-}, \mathfrak{s v e c t}(0 \mid n)\right)_{*}^{m k} \\
& \quad \boxplus \mathbb{C}(a x+b z) \text { if } n>4 \text { or if }(a, b) \notin \mathbb{C}(3,4) \text { and } n=4 .
\end{aligned}
$$

Proof. As in Theorem 6.1, heading 1) follows from a direct calculation based on Lemma 6.1; for the explicit formulas see Appendix 3. Heading 2) is proved in Appendix 2.

Let us clarify the structure of $\left(\mathfrak{h e i}(8 \mid 6), \mathfrak{s v e c t}_{3,4}(4)\right)_{*}^{k}$. This Lie superalgebra is contained in $\mathfrak{u}=\mathfrak{k}(9 \mid 6)$. In sec. 6.3 we have already described the Lie superalgebra $\mathfrak{g}=\tilde{\mathfrak{c}}\left(\mathfrak{s} \mathfrak{e}^{\circ}(4 ; 4)\right)$ and its embedding $i_{1}: \mathfrak{g} \rightarrow \mathfrak{u}$.

Observe that $\mathfrak{g} \supset \mathfrak{a s}$ and this embedding preserves the $\mathbb{Z}$-grading described in sec. 2.1:

$$
\begin{gathered}
\mathfrak{a s}_{-2}=\mathfrak{g}_{-2} ; \quad \mathfrak{a s}_{-1}=\mathfrak{g}_{-1}=\Pi\left(\Lambda^{2}\left(\xi_{1}, \xi_{2}, \xi_{3}, \xi_{4}\right)\right) \\
\mathfrak{a s}_{0}=\mathfrak{s l}(4) \subset \mathfrak{g}_{0}=\mathfrak{s} \mathfrak{v e c t}(0 \mid 4) ; \quad \mathfrak{a s}_{1}=\Pi\left(S^{2}\left(q_{1}, q_{2}, q_{3}, q_{4}\right)\right) \subset \mathfrak{g}_{1} .
\end{gathered}
$$

For the role of $\mathfrak{h}$ (see 4.3 and 6.3) take $\mathfrak{k} \mathfrak{a s}$. It follows from Theorem 3.2 that $\mathfrak{a s} \subset \mathfrak{k a s}$; set $\tilde{\mathfrak{h}}=\mathfrak{a s}$. Let $R: \tilde{\mathfrak{h}} \longrightarrow \mathfrak{g}$ be the embedding that executes the isomorphism of two copies of $\mathfrak{a s}$. (Notice that $R$ preserves the $\mathbb{Z}$-grading (1.1) of ass.) The map $i_{2}=i_{1} R$ determines an embedding of $\tilde{\mathfrak{h}}$ into $\mathfrak{k}(9 \mid 6)$.

But $\mathfrak{h}_{0}=\tilde{\mathfrak{h}}_{0} \oplus \mathbb{C} K_{t}$. It turns out that $i_{2}$ can be extended to an embedding $i_{2}: \mathfrak{k a s} \longrightarrow \mathfrak{u}$ and $i_{1}\left(\tilde{\mathfrak{c}}\left(\mathfrak{s l e}{ }^{\circ}(4 ; 4)\right)\right) \cap i_{2}(\mathfrak{k a s})=\mathfrak{a s}$.

As in the above examples, we have: $i_{1}\left(\mathfrak{g}_{-} \oplus \mathfrak{g}_{0}\right)+i_{2}\left(\mathfrak{h}_{-} \oplus \mathfrak{h}_{0}\right) \cong \mathfrak{u}_{-} \boxplus \mathbb{C}(3 x+4 z)$ (the sum in the left hand side here is not a direct one!). But, unlike the cases $\mathfrak{v} \mathfrak{l}(4 \mid 3)$ and $\mathfrak{m} \mathfrak{b}(4 \mid 5)$, the nondirect sum of $\mathfrak{g}=\tilde{\mathfrak{c}}\left(\mathfrak{s} \mathfrak{l} \mathfrak{e}^{\circ}(4 ; 4)\right)$ with $\mathfrak{h}=\mathfrak{k} \mathfrak{s} \mathfrak{s}$ does not span the whole of $\mathfrak{k} \mathfrak{s l e}(9 \mid 6)$. A description similar to the cases $\mathfrak{v} \mathfrak{l}(4 \mid 3)$ and $\mathfrak{m} \mathfrak{b}(4 \mid 5)$ will be given in $\S 7$. 


\section{The WeIsfeiler REgRAdings}

7.1. Weisfeiler regradings. Let $\mathfrak{g}=\bigoplus_{j \geq-d} \mathfrak{g}_{j}$ be a $\mathbb{Z}$-graded vectorial superalgebra. By a Weisfeiler regrading or $W$-regrading of $\mathfrak{g}$ we understand a second structure of $\mathbb{Z}$-graded superalgebra $\mathfrak{g}=\bigoplus_{i>-D} \mathfrak{h}_{i}$ (with $D<\infty$ ) such that

1) the two gradings determine a bigrading: $\mathfrak{h}_{i}=\bigoplus_{j} \mathfrak{g}_{j} \cap \mathfrak{h}_{i}$;

2) $\mathfrak{h}^{0}=\bigoplus_{j \geq 0} \mathfrak{h}_{j}$ is a maximal Lie subalgebra of finite codimension; and

3 ) the grading $\mathfrak{h}$ is transitive: for any non-zero $x \in \mathfrak{h}_{k}$ for $k \geq 0$ there is $y \in \mathfrak{h}_{-1}$ such that $[x, y] \neq 0$.

In what follows in this section $\mathfrak{h}$ denotes a Lie superalgebra obtained from $\mathfrak{g}$ by a regrading. We will describe all the $\mathrm{W}$-regradings of the exceptional Lie superalgebras.

7.1.1. Lemma. Let $\mathfrak{h}=\bigoplus_{i \geq-D} \mathfrak{h}_{i}$ be a W-regrading of the Lie superalgebra $\mathfrak{g}=$ $\bigoplus_{j \geq-d} \mathfrak{g}_{j}$. It determines a bigrading:

$$
\mathfrak{h}_{i}=\bigoplus_{j \geq m(i)}^{M(i)} \mathfrak{h}_{i, j}, \text { where } \mathfrak{h}_{i, j}=\mathfrak{h}_{i} \cap \mathfrak{g}_{j}
$$

Suppose $\operatorname{dim}\left(\mathfrak{h}_{0} \cap \mathfrak{g}_{-}\right)=0 \mid k$. Then the number $M=M(-1)-m(-1)+1$ of the summands $\mathfrak{h}_{-1, j}$ in the decomposition (7.1.1) of $\mathfrak{h}_{-1}$ does not exceed the number of homogeneous components with respect to the $\mathbb{Z}$-grading of $\Lambda\left(\mathfrak{h}_{0} \cap \mathfrak{g}_{-}\right)$.

In particular, if $\mathfrak{h}_{0} \cap \mathfrak{g}_{-}$is homogeneous with respect to the $\mathbb{Z}$-grading in $\mathfrak{g}$, i.e., $\mathfrak{h}_{0} \cap \mathfrak{g}_{-} \subset \mathfrak{g}_{j}$ for some $j$, then $M \leq k+1$.

Proof. The component $V=\mathfrak{h}_{-1, M(-1)}$ of the maximal degree is invariant with respect to the subalgebra $\mathfrak{h}_{0, \geq 0}=\bigoplus_{j \geq 0} \mathfrak{h}_{0, j}$. Since the $\mathfrak{h}_{0}$-module $\mathfrak{h}_{-1}$ is irreducible, it must be a quotient of the induced module: as spaces, $\operatorname{ind}_{\mathfrak{h}_{0, \geq 0}}^{\mathfrak{h}_{0}} V \cong \Lambda\left(\mathfrak{h}_{0} \cap \mathfrak{g}_{-}\right) \otimes$ $V$.

7.1.2. Lemma. If $\mathfrak{h}_{0} \cap \mathfrak{g}_{-}=0$, then $\mathfrak{h}_{0} \subset \mathfrak{g}_{0}$ and $\mathfrak{h}_{-1}$ is homogeneous.

Proof. Clearly, $\mathfrak{h}_{0}=\bigoplus_{k>0} \mathfrak{h}_{0, k}$ and $\mathfrak{h}_{-1}=\bigoplus_{j} \mathfrak{h}_{-1, j}$.

The Lie superalgebra $\mathfrak{h}_{0,0}$ transforms $\mathfrak{h}_{-1, j}$ into itself and the operators from $\mathfrak{h}_{0, k}$ send $\mathfrak{h}_{-1, j}$ into $\mathfrak{h}_{-1, j+k}$. Therefore, if the representation of $\mathfrak{h}_{0}$ on $\mathfrak{h}_{-1}$ is irreducible, then $\mathfrak{h}_{-1}$ is homogeneous with respect to the grading of $\mathfrak{g}$, i.e., $\mathfrak{h}_{-1}=\mathfrak{h}_{-1, j_{0}}$ for some $j_{0}$. But then $\mathfrak{h}_{0, k}$ for $k>0$ sends $\mathfrak{h}_{-1}$ to 0 . Since $\mathfrak{h}$ is transitive, $\mathfrak{h}_{0, k}=0$ for all $k>0$, i.e., $\mathfrak{h}_{0}=\mathfrak{h}_{0,0}$.

7.1.3. Lemma. If $\mathfrak{h}_{0} \cap \mathfrak{g}_{-}=0$ and there exists a nonzero $x \in \mathfrak{g}_{-1} \cap \mathfrak{h}_{-}$, then the gradings of $\mathfrak{h}$ and $\mathfrak{g}$ coincide.

Proof. $\mathfrak{h}_{-1}=\mathfrak{h}_{-1, j_{0}} \subset \mathfrak{g}_{j_{0}}$ and, therefore, $\mathfrak{h}_{-k} \subset \mathfrak{g}_{k \cdot j_{0}}$. If $x \in \mathfrak{h}_{-k}$, then $-1=k \cdot j_{0}$, implying $k=1$ and $j_{0}=-1$, i.e., $\mathfrak{h}_{-1} \subset \mathfrak{g}_{-1}$, and, therefore, $\mathfrak{h}_{-1}=\mathfrak{g}_{-1}$ and $\mathfrak{h}_{i}=\mathfrak{g}_{i}$ for all $i$.

7.2. How to describe all the $\mathbf{W}$-regradings. 1) Determine $\mathfrak{h}_{0} \cap \mathfrak{g}_{-1}$.

2) Construct a "minimal" (i.e., most tightly compressed) regrading with the given intersection, i.e., such that it preserves in $\mathfrak{h}_{-1}$ all the elements of $\mathfrak{g}_{-1}$ except for those that have to go away in view of the condition on the intersection.

3 ) If the "minimal" regrading is a Weisfeiler one, then with the help of Lemmas 7.1.2 and 7.1.3 we prove that any other $\mathrm{W}$-regrading with the given intersection coincides with the minimal one. 
4) If the "minimal" regrading is not a Weisfeiler one, then with the help of Lemma 7.1.1 we prove that there are no $\mathrm{W}$-regradings with the given intersection.

7.3. W-regradings of $\mathfrak{g}=\mathfrak{v} \mathfrak{e}(4 \mid 3)$. We consider a realization of $\mathfrak{g}$ by a nondirect sum $i_{1}(\mathfrak{l} \mathfrak{e}(3 ; 3))+i_{2}(\mathfrak{l} \mathfrak{e}(3))$; cf. sec. 4.3 .

Notations: $\xi_{i}^{\prime}=i_{1}\left(\xi_{i}\right), u_{i}^{\prime}=i_{1}\left(u_{i}\right) ; \xi_{i}=i_{2}\left(\xi_{i}\right), u_{i}=i_{2}\left(u_{i}\right)$ for $i=1,2,3$.

Then

$$
\mathfrak{g}_{-1}=\operatorname{Span}\left(\xi_{i}=\xi_{i}^{\prime} ; u_{k}=\xi_{i}^{\prime} \xi_{j}^{\prime} \text { for even permutations }(i, j, k) ; \xi_{1}^{\prime} \xi_{2}^{\prime} \xi_{3}^{\prime}\right) .
$$

For any regrading we have:

$$
\begin{array}{ll}
\operatorname{deg} \xi_{i}+\operatorname{deg} u_{i}=N & \text { for any } i=1,2,3 ; \\
\operatorname{deg} \xi_{i}^{\prime}+\operatorname{deg} u_{i}^{\prime}=N^{\prime} & \text { for any } i=1,2,3 ; \\
\operatorname{deg}_{\text {Lie }}(f)=\operatorname{deg}(f)-N ; & \operatorname{deg}_{\text {Lie }}\left(f^{\prime}\right)=\operatorname{deg}\left(f^{\prime}\right)-N^{\prime} .
\end{array}
$$

The conditions $\xi_{i}=\xi_{i}^{\prime}$ imply that

$$
\operatorname{deg} u_{i}^{\prime}=\operatorname{deg} u_{i},
$$

whereas the conditions $u_{k}=\xi_{i}^{\prime} \xi_{j}^{\prime}$ imply that

$\operatorname{deg} u_{k}-N=\operatorname{deg} \xi_{i}^{\prime}+\operatorname{deg} \xi_{j}^{\prime}-N^{\prime} \Longleftrightarrow \operatorname{deg} \xi_{i}^{\prime}=u_{j}^{\prime}-\operatorname{deg} \xi_{k}=\operatorname{deg} u_{j}^{\prime}-\operatorname{deg} \xi_{k}^{\prime}$.

3a) $\mathfrak{h}=\mathfrak{v} \mathfrak{e}(4 \mid 3 ; 1)$. Set

$$
\begin{aligned}
& \operatorname{deg} \xi_{1}=0, \operatorname{deg} u_{1}=2 ; \quad N=2 ; \\
& \operatorname{deg} \xi_{2}=\operatorname{deg} \xi_{3}=\operatorname{deg} u_{2}=\operatorname{deg} u_{3}=1 .
\end{aligned}
$$

Formulas (7.3.2) and (7.3.3) imply that

$$
\begin{aligned}
& \operatorname{deg} \xi_{1}^{\prime}=0, \operatorname{deg} u_{1}^{\prime}=2 ; \quad N^{\prime}=2 ; \\
& \operatorname{deg} \xi_{2}^{\prime}=\operatorname{deg} \xi_{3}^{\prime}=\operatorname{deg} u_{2}^{\prime}=\operatorname{deg} u_{3}^{\prime}=1 .
\end{aligned}
$$

Hence, $\mathfrak{h}=\bigoplus_{i \geq-2} \mathfrak{h}_{i}$, where

$$
\begin{aligned}
\mathfrak{h}_{-2}= & \mathbb{C} \cdot \xi_{1}=\mathbb{C} \cdot \xi_{1}^{\prime} \\
\mathfrak{h}_{-1}= & \operatorname{Span}\left(\xi_{2}, \xi_{3}, u_{2}, u_{3}\right) \otimes \Lambda\left(\xi_{1}\right)=\operatorname{Span}\left(\xi_{2}^{\prime}, \xi_{3}^{\prime}, u_{2}^{\prime}, u_{3}^{\prime}\right) \otimes \Lambda\left(\xi_{1}^{\prime}\right) \\
\mathfrak{h}_{0}= & \left(\Lambda^{2}\left(\xi_{2}, \xi_{3}\right) \bigoplus \Lambda^{1}\left(\xi_{2}, \xi_{3}\right) \otimes S^{1}\left(u_{2}, u_{3}\right) \bigoplus S^{2}\left(u_{2}, u_{3}\right)\right) \otimes \Lambda\left(\xi_{1}\right) \boxplus \operatorname{vect}\left(\xi_{1}\right) \\
& +(\mathrm{NOT} \oplus !) \text { the same with }{ }^{\prime}
\end{aligned}
$$

Observe that since $\operatorname{id}(\mathfrak{s l}(2)) \cong \operatorname{id}^{*}(\mathfrak{s l}(2))$, we have

$$
\begin{gathered}
\Lambda^{2}\left(\xi_{2}, \xi_{3}\right) \bigoplus \Lambda^{1}\left(\xi_{2}, \xi_{3}\right) \otimes S^{1}\left(u_{2}, u_{3}\right) \bigoplus S^{2}\left(u_{2}, u_{3}\right) \\
\cong \mathfrak{p e}(2) \cong \mathfrak{s l}(2) \otimes \Lambda\left(\xi_{1}\right) \boxplus \mathfrak{v e c t}\left(\xi_{1}\right) .
\end{gathered}
$$

Introduce a formal odd indeterminate $\eta$. Set

$$
V=\operatorname{Span}\left(\xi_{2}, \xi_{3}\right) ; \quad \eta V=\operatorname{Span}\left(-u_{3}, u_{2}\right) .
$$

Then $\xi_{1} V=\operatorname{Span}\left(\xi_{1} \xi_{2}, \xi_{1} \xi_{3}\right)$ and $\xi_{1} \eta V=\operatorname{Span}\left(-\xi_{1} u_{3}, \xi_{1} u_{2}\right)$; the action of $\xi_{1} \xi_{2}$ is identical with that of $\frac{\partial}{\partial \eta}$.

In these notations, $\mathfrak{g}_{-1}=V \otimes \Lambda\left(\xi_{1}, \eta\right)=V \otimes \Lambda(2)$ and the bracket on $\mathfrak{g}_{-1}$ is given by the formula:

$$
\left[v_{1} \otimes \varphi_{1}\left(\xi_{1}, \eta\right), v_{2} \otimes \varphi_{2}\left(\xi_{2}, \eta\right)\right]=(-1)^{p\left(\varphi_{1}\right) p\left(v_{2}\right)} \xi_{1} \omega_{v}\left(v_{1}, v_{2}\right) \int \varphi_{1} \varphi_{2} v o l,
$$


where $\omega_{v}$ is a 2 -form on $V$ preserved by $\mathfrak{s l}(2) \cong \mathfrak{s p}(2)$.

The direct calculations show that $\mathfrak{h}_{0} \cong \mathfrak{c}(\mathfrak{s l}(2) \otimes \Lambda(2) \nexists \mathfrak{v e c t}(0 \mid 2))$, where the subalgebra $\mathfrak{v e c t}(0 \mid 2) \subset \mathfrak{g}_{0}$ acts on $\mathfrak{h}_{-1}=V \otimes \Lambda(2)$ as id $\otimes T^{1 / 2}$, where $T^{1 / 2}$ is the representation of $\mathfrak{v e c t}(0 \mid 2)$ in the space of half-densities, $T(\overrightarrow{-1 / 2})$.

Thus,

$$
\mathfrak{v} \mathfrak{e}(4 \mid 3 ; 1)=(\mathfrak{h} \mathfrak{e} \mathfrak{i}(4 \mid 4), \mathfrak{c}(\mathfrak{s l}(2) \otimes \Lambda(2) \boxplus \mathfrak{v e c t}(0 \mid 2)))^{m k} \subset \mathfrak{k}(5 \mid 4) .
$$

3b) $\mathfrak{h}=\mathfrak{v} \mathfrak{k}(4 \mid 3 ; 2)$. Set

$$
\begin{aligned}
& \operatorname{deg} \xi_{1}=\operatorname{deg} \xi_{2}=0, \operatorname{deg} u_{1}=\operatorname{deg} u_{2}=2 ; N=2 ; \\
& \operatorname{deg} \xi_{3}=\operatorname{deg} u_{3}=1
\end{aligned}
$$

Formulas (7.3.2) and (7.3.3) imply then that

$$
\begin{aligned}
& \operatorname{deg} u_{1}^{\prime}=\operatorname{deg} u_{2}^{\prime}=2 ; N^{\prime}=3 ; \\
& \operatorname{deg} \xi_{1}^{\prime}=\operatorname{deg} \xi_{2}^{\prime}=1 ; \operatorname{deg} \xi_{3}^{\prime}=2 ; \operatorname{deg} u_{3}^{\prime}=1 .
\end{aligned}
$$

Hence, $\mathfrak{h}=\bigoplus_{i \geq-2} \mathfrak{h}_{i}$, where

$$
\begin{aligned}
\mathfrak{h}_{-2} & =\operatorname{Span}\left(\xi_{1}=\xi_{1}^{\prime}, \xi_{2}=\xi_{2}^{\prime}, \xi_{1} \xi_{2}=u_{3}^{\prime}\right) \cong \Pi(\Lambda(2) / \mathbb{C} \cdot 1) ; \\
\mathfrak{h}_{-1} & =\operatorname{Span}\left(\xi_{3}, u_{3}\right) \otimes \Lambda\left(\xi_{1}, \xi_{2}\right)=V \otimes \Lambda(2), \operatorname{dim} V=1 \mid 1 ; \\
\mathfrak{h}_{0} & =\mathfrak{c}(\mathfrak{p e}(1) \otimes \Lambda(2) \nexists \mathfrak{v e c t}(0 \mid 2)) .
\end{aligned}
$$

The action of $\mathfrak{h}_{0}$ in $\mathfrak{h}_{-1}$ is not irreducible; hence, $\mathfrak{v} \mathfrak{k}(4 \mid 3 ; 2)$ is not a W-regrading. $3 \mathrm{c}) \mathfrak{h}=\mathfrak{v} \mathfrak{e}(4 \mid 3 ; 3)$. Set

$$
\operatorname{deg} u_{i}=2 ; \operatorname{deg} \xi_{i}=0 ; \operatorname{deg} u_{i}^{\prime}=\operatorname{deg} \xi_{i}^{\prime}=1 ; \quad N=N^{\prime}=2 .
$$

By sec. $4.3, \mathfrak{h}=\mathfrak{v l e}(4 \mid 3 ; 3) \cong \mathfrak{v} \mathfrak{l}(4 \mid 3)$.

$3 \mathrm{~d}) \mathfrak{g}=\mathfrak{v} \mathfrak{e}(4 \mid 3 ; K)$. Set

$$
\operatorname{deg} u_{i}=\operatorname{deg} u_{i}^{\prime}=2 ; \operatorname{deg} \xi_{i}=\operatorname{deg} \xi_{i}^{\prime}=1 ; \quad N=N^{\prime}=3 .
$$

Hence, $\mathfrak{h}=\bigoplus_{i \geq-2} \mathfrak{h}_{i}$, where

$$
\begin{aligned}
\mathfrak{h}_{-2} & =\Pi\left(\operatorname{Span}\left(\xi_{i}=\xi_{i}^{\prime}: i=1,2,3\right)\right) \\
\mathfrak{h}_{-1} & =\Pi\left(\operatorname{Span}\left(u_{i}=\xi_{j}^{\prime} \xi_{k}^{\prime}, \xi_{i} \xi_{j}=u_{k}^{\prime} \text { for all even permutations }(i, j, k)\right)\right. \\
\mathfrak{h}_{0} & =\Pi\left(\operatorname{Span}\left(u_{i} \xi_{j}=u_{i}^{\prime} \xi_{j}^{\prime}\right) \cap \mathfrak{s l e}(3)\right. \\
& \left.\bigoplus \operatorname{Span}\left(\xi_{1} \xi_{2} \xi_{3}, \xi_{1}^{\prime} \xi_{2}^{\prime} \xi_{3}^{\prime}, \sum u_{i} \xi_{i}-\sum u_{i}^{\prime} \xi_{i}^{\prime}\right) \bigoplus \mathbb{C}\left(\sum u_{i} \xi_{i}+\sum u_{i}^{\prime} \xi_{i}^{\prime}\right)\right) \\
& \cong \mathfrak{s l}(3) \oplus \mathfrak{s l}(2) \oplus \mathbb{C} \cdot z .
\end{aligned}
$$

The $\mathfrak{h}_{0}$-action in $\mathfrak{h}_{-1}$ is $\operatorname{id}(\mathfrak{s l}(3)) \otimes \operatorname{id}(\mathfrak{s l}(2)) \otimes 1$.

7.3.1. Statement. Any W-regrading of $\mathfrak{g}=\mathfrak{v} \mathfrak{e}(4 \mid 3)$ is either $\mathfrak{v l e}(4 \mid 3 ; 1)$ or $\mathfrak{v} \mathfrak{k}(4 \mid 3 ; K)$.

Proof. Let $\mathfrak{h}=\bigoplus_{i \geq-d} \mathfrak{h}_{i}$ be a W-regrading of $\mathfrak{g}$. Since the elements $\xi_{i}=\xi_{i}^{\prime}$ act in $\mathfrak{g}$ as $\frac{\partial}{\partial u_{i}}$, all of them must lie in $\mathfrak{h}_{-}$. Set $U=\operatorname{Span}\left(u_{1}, u_{2}, u_{3}\right)$ and let $\operatorname{dim} U \cap \mathfrak{h}_{0}=m$, where $0 \leq m \leq 3$.

If $m=0$, then by Lemma 7.1.2 the gradings in $\mathfrak{g}$ and $\mathfrak{h}$ can differ only if $\xi_{1}^{\prime} \xi_{2}^{\prime} \xi_{3}^{\prime} \in$ $\mathfrak{h}_{0}$. But then, since $\mathfrak{h}_{0} \not \supset u_{k}=\xi_{i}^{\prime} \xi_{j}^{\prime}=\left[u_{k}^{\prime}, \xi_{1}^{\prime} \xi_{2}^{\prime} \xi_{3}^{\prime}\right]$, it follows that $u_{k}^{\prime}=\xi_{i} \xi_{j} \notin \mathfrak{h}_{0}$.

But then $\mathfrak{h}$ and $\mathfrak{g}=\mathfrak{v} \mathfrak{k}(4 \mid 3 ; K)$ satisfy the conditions of Lemma 7.1.3; hence, their gradings coincide. 
If $m=1$, then, up to linear automorphisms, we may assume that $U \cap \mathfrak{h}_{0}=\mathbb{C} \cdot u_{1}$. Since ad $u_{1}$ acts as $\frac{\partial}{\partial \xi_{1}}$, we deduce that $\xi_{1} \xi_{2}, \xi_{2} \xi_{3}, \xi_{1} u_{2}, \xi_{1} u_{3} \notin \mathfrak{h}_{0}$. So we can apply Lemma 7.1.2 to $\mathfrak{h}$ and $\mathfrak{g}=\mathfrak{v} \mathfrak{e}(4 \mid 3 ; 1)$.

If $m=2$, then, up to linear automorphisms, we may assume that $U \cap \mathfrak{h}_{0}=$

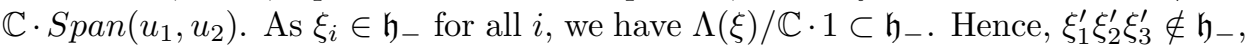
as an element dual to $\xi_{1} \xi_{2} \xi_{3} \in \mathfrak{h}_{-}$.

If $\mathfrak{h} \neq \mathfrak{v} \mathfrak{l e}(4 \mid 3 ; 2)$, then $\mathfrak{h}_{-} \neq \mathfrak{v} \mathfrak{v e}(4 \mid 3 ; 2)_{-}$. Hence, either $\mathfrak{h}=\Lambda(\xi) / \mathbb{C} \cdot 1$, or $g \otimes \Lambda\left(\xi_{1}, \xi_{2}\right) \in \mathfrak{h}_{\text {- for some }} g \in \mathfrak{g}_{\geq 0}$.

In the first case, the subspases $\Lambda\left(\xi_{1}, \xi_{2}\right) / \mathbb{C} \cdot 1$ and $\xi_{3} \otimes \Lambda\left(\xi_{1}, \xi_{2}\right)$ cannot belong to the same component $\mathfrak{h}_{-k}$ because of $u_{3} \notin \mathfrak{h}_{0}$. But none of these subspaces generates the other. Thus, $\mathfrak{h}$ is not a $\mathrm{W}$-regrading.

In the second case, either $\mathfrak{h}_{-1}$ does not generate $\mathfrak{h}_{-}$, or $\mathfrak{h}_{-1}$ contradicts Lemma 7.1.1. In both cases, $\mathfrak{h}$ is not a $\mathrm{W}$-regrading.

If $m=3$, then $\xi_{i} \notin \mathfrak{h}_{0}$. Hence, $\Lambda\left(\xi_{1}, \xi_{2}, \xi_{3}\right) / \mathbb{C} \cdot 1 \cap \mathfrak{h}_{0}=0$. So we can apply Lemma 7.1.3 to $\mathfrak{h}$ and $\mathfrak{g}=\mathfrak{v} \mathfrak{k}(4 \mid 3 ; 3)=\mathfrak{v} \mathfrak{e}(4 \mid 3)$.

7.4. W-regradings of $\mathfrak{g}=\mathfrak{m} \mathfrak{b}(4 \mid 5)$. We consider a realization of $\mathfrak{g}$ by a nondirect $\operatorname{sum}\left(i_{1}\left(\mathfrak{b}_{1 / 2}(3 ; 3)\right)+i_{2}\left(\mathfrak{b}_{-3,1}(3)\right)\right.$; cf. sec. 6.3 .

Recall that if $u_{1}, u_{2}, u_{3}, \xi_{1}, \xi_{2}, \xi_{3}$ and $\tau$ are the standard indeterminates in $\mathfrak{m}(3)$, then each generating function of $\mathfrak{b}_{1 / 2}(3 ; 3)$ is of the form

$$
F=f+\frac{1}{\operatorname{deg}(\Delta(f))+1} \tau \Delta(f), \text { where } f \in \mathbb{C}[u, \xi] .
$$

Therefore, we may use $f$ instead of $F$ and $f^{\prime}$ instead of $F^{\prime}$. Thus, $\mathfrak{g}_{-}=\mathfrak{g}_{-2} \oplus \mathfrak{g}_{-1}$, where

$$
\begin{aligned}
& \mathfrak{g}_{-2}=\Pi(\mathbb{C} \cdot 1), \\
& \mathfrak{g}_{-1}=\Pi\left(\operatorname{Span}\left(1^{\prime}, \xi_{i}=\xi_{i}, u_{i}=\xi_{j}^{\prime} \xi_{k}^{\prime}, \xi_{1}^{\prime} \xi_{2}^{\prime} \xi_{3}^{\prime}\right)\right) .
\end{aligned}
$$

4 a) $\mathfrak{h}=\mathfrak{m} \mathfrak{b}(4 \mid 5 ; 1)$. Then $\mathfrak{h}$ is described by (7.3.4)-(7.3.4). Moreover, the superspaces $\mathfrak{h}_{-1}$ and $\mathfrak{h}_{0}$ are the same as for $\mathfrak{v} \mathfrak{k}(4 \mid 3 ; 1)$ whereas our component $\mathfrak{h}_{-2}$ is spanned by $\mathfrak{v} \mathfrak{l}(4 \mid 3 ; 1)_{-2}=\operatorname{Span}\left(\xi_{1}\right)$ together with 1 and $1^{\prime}$.

To visualize the result better, redenote the elements of $\mathfrak{h}_{-2}$ :

$$
1 \mapsto \eta, 1^{\prime} \mapsto \xi_{1}, \xi_{1} \mapsto \xi_{1} \eta .
$$

Then the bracket in $\mathfrak{h}_{-1}$ is of the form

$$
\left[v_{1} \otimes \varphi_{1}\left(\xi_{1}, \eta\right), v_{2} \otimes \varphi_{2}\left(\xi_{1}, \eta\right)\right]=(-1)^{p\left(\varphi_{1}\right) p\left(\varphi_{2}\right)} \omega_{v}\left(v_{1}, v_{2}\right) \cdot \varphi_{1} \varphi_{2} .
$$

Thus, with the natural action of vector fields on the functions, we have

$$
\mathfrak{h}_{-} \cong(\mathfrak{h e i}(2 \mid 0) \otimes \Lambda(2)) / \mathbb{C} \cdot z \otimes 1 \text { and } \mathfrak{h}_{0} \cong \mathfrak{c}(\mathfrak{s l}(2) \otimes \Lambda(2) \boxplus \mathfrak{v e c t}(0 \mid 2)) .
$$

Observe that the only difference of nonpositive parts of $\mathfrak{m} \mathfrak{b}(4 \mid 5 ; 1)$ and $\mathfrak{h}(2 \mid 4 ; 2)$ is that the former has one element more - the center - in the component of degree 0 . Thus,

4b) $\mathfrak{g}=\mathfrak{m} \mathfrak{b}(4 \mid 5 ; 2)$. Relations $(7.3 .9)-\left(7.3 .9^{\prime}\right)$ mean that $1 \in \mathfrak{h}_{-2}$ while $1^{\prime} \in \mathfrak{h}_{-3}$.

$$
\mathfrak{h}_{-3}=\mathbb{C} \cdot 1^{\prime} ; \mathfrak{h}_{-2}=\Pi(\Lambda(2)) ; \mathfrak{h}_{-1}=V \otimes \Lambda(2) .
$$

Observe that $\operatorname{dim} V=1 \mid 1$, so $V \cong \Lambda(1)$; hence, $V \otimes \Lambda(2) \cong \Lambda(1) \otimes \Lambda(2) \cong \Lambda(3)$ with the action of $\mathfrak{p e}(1) \cong T^{1 / 2}(\mathfrak{v e c t}(0 \mid 1))$ on $V$. 
The bracket $\left[\mathfrak{h}_{-1}, \mathfrak{h}_{-2}\right]$ is given by the formula

$$
\left[\varphi_{1}\left(\xi_{3} ; \xi_{1}, \xi_{2}\right), \varphi_{2}\left(\xi_{1}, \xi_{2}\right)\right]=c \cdot \int \varphi_{1} \varphi_{2} \operatorname{vol}\left(\xi_{1}, \xi_{2}, \xi_{3}\right)
$$

Thus,

$$
\mathfrak{h}_{0} \cong \mathfrak{c}\left(T^{1 / 2}(\mathfrak{v e c t}(0 \mid 1)) \otimes \Lambda(2) \boxplus \mathfrak{v e c t}(0 \mid 2)\right) .
$$

Since the $\mathfrak{h}_{0}$-action on $\mathfrak{h}_{-1}$ is reducible, this is not a W-filtration.

$4 c) \mathfrak{m b}(4 \mid 5 ; 3)$. The regrading is determined by $(7.3 .10)$. By sec. $6.3 \mathfrak{m b}(4 \mid 5 ; 3) \cong$

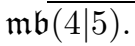

$4 d) \mathfrak{m b}(4 \mid 5 ; K)$. Relations (7.3.11) mean that $\mathfrak{h}_{-3}=\operatorname{Span}\left(1,1^{\prime}\right)$. The other nonpositive components are the same as for $\mathfrak{v l e}(4 \mid 3 ; K)$.

Statement. Any $W$-regrading of $\mathfrak{m b}(4 \mid 5)$ is isomorphic to $\mathfrak{m b}(4 \mid 5 ; 1)$ or to $\mathfrak{m b}(4 \mid 5 ; K)$.

Proof is similar to that of Statement 7.3.1.

7.5. Statement. $\mathfrak{g}=\mathfrak{v a \mathfrak { s }}(4 \mid 4)$ has no $W$-regradings.

Proof. As in Theorem 2.3, realize the elements of $\mathfrak{g}=\mathfrak{v a s}(4 \mid 4)$ in the form

$$
D_{f}+c \cdot Z \text {, where } f \in \mathfrak{s l e}^{\circ}(4) .
$$

Suppose $\mathfrak{h}=\bigoplus_{i>-d} \mathfrak{h}_{i}$ is a W-regrading of $\mathfrak{g}$ distinct from the initial one. By Lemmas 7.1.2 and 7.1.3 we see that $\mathfrak{h}_{0} \cap \mathfrak{g}_{-1} \neq 0$. Observe that if $\operatorname{deg}_{\xi} f \leq 1$, then $D_{f}=L e_{f}$. In particular, $\left[D_{f(u)}, D_{\xi_{i}}\right]=\frac{\partial f}{\partial u_{i}}$. Hence, $D_{\xi_{i}} \in \mathfrak{h}_{-}$for all $i=1, \ldots, 4$; hence, $V=\operatorname{Span}\left(D_{q_{i}}: i=1, \ldots, 4\right) \cap \mathfrak{h}_{0} \neq 0$.

Lemma. $\operatorname{dim} V \leq 1$.

Proof. If $\operatorname{dim} V>1$, then, up to linear transformations, we may assume that at least $D_{u_{1}}, D_{u_{2}} \in \mathfrak{h}_{0}$. But $\left[D_{u_{i}}, D_{\xi_{i} \xi_{j}}\right]=D_{u_{j}}$ and, therefore, $D_{\xi_{1} \xi_{i}}, D_{\xi_{2} \xi_{j}} \in \mathfrak{h}_{-}$ for any $i=2,3,4$ and $j=1,3,4$. In particular, $D_{\xi_{1} \xi_{4}}, D_{\xi_{2} \xi_{3}} \in \mathfrak{h}_{-}$. But $\left[D_{\xi_{1} \xi_{4}}, D_{\xi_{2} \xi_{3}}\right]=\lambda Z \in \mathfrak{h}_{-}$.

Since ad $Z$ determines the $\mathbb{Z}$-grading of $\mathfrak{h}$, the element $Z$ belongs to $\mathfrak{h}_{0}$. The contradiction obtained completes the proof of Lemma.

Let us continue with the proof of Statement. It remains to consider the case $\operatorname{dim} V=1$. Let, for definiteness, $D_{u_{1}} \in \mathfrak{h}_{0}$, whereas $D_{u_{i}} \notin \mathfrak{h}_{0}$ for $i \neq 1$.

In principle, such regradings are possible: for example, for $\mathfrak{v a s}(4 \mid 4 ; 1)$ we set $\operatorname{deg} \xi_{1}=0, \operatorname{deg} u_{1}=3 ; \operatorname{deg} \xi_{i}=2, \operatorname{deg} u_{i}=1$ for $i>1$. We see that $\mathfrak{h}_{-3}=\mathbb{C} D_{\xi_{1}}$; $\mathfrak{h}_{-2}=\operatorname{Span}\left(D_{\xi_{1} q_{i}}, D_{u_{i}} ; i>1\right) ;$ and $\mathfrak{h}_{-1}=\mathfrak{v a s}(4 \mid 4 ; 1)_{-1}$ contains three components of the bidegree:

$$
\begin{aligned}
\mathfrak{h}_{-1,-1}=\operatorname{Span}\left(D_{\xi_{i}} ; i>1\right), \mathfrak{h}_{-1,0} & =\operatorname{Span}\left(D_{\xi_{1} \xi_{i}} ; i>1\right), \mathfrak{h}_{-1,1} \\
& =\operatorname{Span}\left(D_{S^{2}}\left(u_{i} ; i>1\right) \otimes \Lambda\left(\xi_{1}\right)\right)
\end{aligned}
$$

by Lemma $7.1 .1 \mathfrak{v a s}(4 \mid 4 ; 1)$ is not a W-grading.

Let $\mathfrak{h}=\bigoplus_{i>-d} \mathfrak{h}_{i}$ be a W-regrading of $\mathfrak{g}=\mathfrak{v} \mathfrak{a} \mathfrak{s}(4 \mid 4)$ distinct from $\mathfrak{v a s}(4 \mid 4 ; 1)$ but such that $D_{u_{1}} \in \mathfrak{h}_{0}$. Let $D_{\xi_{i}} \in \mathfrak{h}_{-n_{i}}$ for $n_{i} \geq 1$. Then $D_{\xi_{1} \xi_{i}} \in \mathfrak{h}_{-n_{i}}$ but

$$
D_{\xi_{1} \xi_{i}}=\xi_{1} \frac{\partial}{\partial u_{i}}-\xi_{i} \frac{\partial}{\partial u_{1}}+\lambda\left(u_{j} \frac{\partial}{\partial \xi_{k}}-u_{k} \frac{\partial}{\partial \xi_{j}}\right)
$$


implying $D_{u_{j}} \in \mathfrak{h}_{-n_{i}-n_{k}}$. Similarly, $D_{u_{i} u_{j}} \in \mathfrak{h}_{-n_{k}}$. Thus, all the elements of the form $D_{\xi_{1}}, D_{\xi_{i}}$, and $D_{u_{i}}, D_{\xi_{1} \xi_{i}}$, as well as $D_{u_{i} u_{j}}$ and $D_{\xi_{1} u_{i} u_{j}}$ with $i>1$ belong to $\mathfrak{h}_{-}$.

Observe that neither $D_{\xi_{1}}$ nor $D_{u_{i}}$ can belong to $\mathfrak{h}_{-1}$. But $\mathfrak{h}_{-1}$ generates the whole of $\mathfrak{h}_{-}$. Hence,

1) $D_{\xi_{i}} \in \mathfrak{g}_{-1}$ for at least one $i$ (and then $D_{\xi_{1} \xi_{i}} \in \mathfrak{h}_{-1}$ and $D_{\xi_{1} \xi_{i}} \in \mathfrak{g}_{0}$ ); and

2) $\mathfrak{h}_{-1}$ contains at least one element from $\mathfrak{g}_{>0}=\bigoplus_{i>0} \mathfrak{g}_{i}$ : otherwise we cannot obtain $D_{\xi_{1} u_{i} u_{j}} \in \mathfrak{g}_{1} \cap \mathfrak{h}_{-}$.

By Lemma 7.1.1 $\mathfrak{h}$ is not a W-regrading.

7.6. W-regradings of $\mathfrak{g}=\mathfrak{k} \mathfrak{a} \mathfrak{s} \subset \mathfrak{k}(1 \mid 6)$. Clearly, the regradings of $\mathfrak{k}(1 \mid 6)$ induce the regradings of $\mathfrak{k} \mathfrak{a}$. However, due to nonsymmetry of $\mathfrak{k} \mathfrak{a s}$ inside of $\mathfrak{k}(1 \mid 6)$ isomorphic regradings of $\mathfrak{k}(1 \mid 6)$ obtained by the replacement $\xi \longleftrightarrow \eta$ may produce distinct regradins of $\mathfrak{k} \mathfrak{a} \mathfrak{s}$. For definiteness, fix a realization: $\mathfrak{k a s}=\mathfrak{k a s}^{\xi}$.

6a) $\mathfrak{k}(1 \mid 6 ; 1)$ induces two regradings of $\mathfrak{k} \mathfrak{a}$ that we will shorthand as $1 \xi$ and $1 \eta$ : with the degree of one of the $\xi$ 's (resp. $\eta$ 's) set equal to zero, e.g.:

$$
\begin{aligned}
\mathfrak{k} \mathfrak{a} \mathfrak{s}(; 1 \xi): & \operatorname{deg} \xi_{1}=0, \operatorname{deg} \eta_{1}=\operatorname{deg} t=2, \\
& \operatorname{deg} \xi_{i}=\operatorname{deg} \eta_{i}=1 \text { for } i>1 .
\end{aligned}
$$

Set $\operatorname{deg} K_{f}=\operatorname{deg} f-2$. We see that $\mathfrak{k}(1 \mid 6 ; 1)_{-} \subset \mathfrak{k} \mathfrak{a}(; 1 \xi)_{-}$which means that $\mathfrak{k}(1 \mid 6 ; 1)_{-}=\mathfrak{k} \mathfrak{a s}(; 1 \xi)_{-}$. Moreover,

$$
\begin{aligned}
& \mathfrak{k}(1 \mid 6 ; 1)_{0} \subset \mathfrak{k}(1 \mid 6)_{-1} \oplus \mathfrak{k}(1 \mid 6)_{0} \oplus \mathfrak{k}(1 \mid 6)_{1} ; \\
& \mathfrak{k}(1 \mid 6 ; 1)_{0} \cap \mathfrak{k}(1 \mid 6)_{1}=\left(\mathbb{C} t \oplus \Lambda^{2}\left(\xi_{2}, \eta_{2}, \xi_{3}, \eta_{3}\right)\right) \otimes \xi_{1} .
\end{aligned}
$$

From the explicit description of $\mathfrak{k a s}_{1}$ (see (3.3.1)) we derive that $K_{t \xi_{1}} \in \mathfrak{k a s}$ and of the 6 elements of $\Lambda^{2}\left(\xi_{2}, \eta_{2}, \xi_{3}, \eta_{3}\right) \otimes \xi_{1}$ only 3 belong to $\mathfrak{k a s}$; on the space they span, $\mathfrak{o}(4) \cong \mathfrak{s l}(2) \oplus \mathfrak{s l}(2)$ acts as ad $\otimes \chi_{0}$, where $\chi_{0}$ is the trivial character. Finally,

$$
\begin{aligned}
& \mathfrak{k} \mathfrak{a} \mathfrak{s}(; 1 \xi)_{-1}=\operatorname{id}(\mathfrak{s l}(2)) \otimes \operatorname{id}(\mathfrak{g l} l(2)) \otimes \Lambda(1) ; \\
& \mathfrak{k a s}(; 1 \xi)_{0}=\mathfrak{s l}(2) \oplus \mathfrak{g l}(2) \boxplus \mathfrak{v e c t}(0 \mid 1) .
\end{aligned}
$$

Similarly, we prove that

$$
\mathfrak{k a s}(; 1 \xi) \cong \mathfrak{k a s}(; 1 \eta)
$$

Under this isomorphism the two copies of $\mathfrak{s l}(2)$ constituting $\mathfrak{o}(4)$ change places. We denote these Lie superalgebras uniformly: $\mathfrak{k} \mathfrak{a}(; 1)$.

$6 \mathrm{~b}) \mathfrak{g}=\mathfrak{k}(1 \mid 6 ; 2)$ induces three regradings of $\mathfrak{k} \mathfrak{a s}$ that we will shorthand as $2 \xi$, $1 \xi+1 \eta$ and $2 \eta$. To describe them, consider, first, the nonpositive terms of $\mathfrak{k}(1 \mid 6 ; 2)$ :

\begin{tabular}{|c|c|c|}
\hline-2 & -1 & 0 \\
\hline$\Lambda(2)$ & $V_{2} \otimes \Lambda(2)$ & $\mathfrak{c o}(2) \otimes \Lambda(2) \boxplus \mathfrak{v e c t}(0 \mid 2)$ \\
\hline
\end{tabular}

Here $\operatorname{dim} V_{2}=0 \mid 2$ and the $\mathfrak{g}_{0}$-action in $\mathfrak{g}_{-1}$ is reducible.

In each of the cases $\mathfrak{h}=\mathfrak{k} \mathfrak{a} \mathfrak{s}(; 2 \xi)$ and $\mathfrak{k} \mathfrak{a s}(; 2 \eta)$ and $\mathfrak{k} \mathfrak{a s}(; 1 \xi+1 \eta)$ their $\mathfrak{h}_{-2}$ are identical with $\mathfrak{g}_{-2}$ and $\mathfrak{h}_{0}$ is the subalgebra of $\mathfrak{g}_{0}$ preserving $\mathfrak{h}_{-1}$. So let us describe $\mathfrak{h}_{-1}$. It is obtained from $\mathfrak{g}_{-1}$ by deleting one element:

Case $2 \xi . \mathfrak{h}_{-2}=\Lambda\left(\xi_{1}, \xi_{2}\right)$,

$$
\begin{aligned}
\mathfrak{h}_{-1} & =\xi_{3} \otimes \Lambda\left(\xi_{1}, \xi_{2}\right) \bigoplus \eta_{3} \otimes\left(1 \otimes \Lambda\left(\xi_{1}, \xi_{2}\right)\right) \cong \Pi\left(\Lambda\left(\xi_{1}, \xi_{2}\right)\right) \bigoplus \mathbb{C}^{2 \mid 1} ; \\
\mathfrak{h}_{0} & =(t+\Phi) \otimes \Lambda\left(\xi_{1}, \xi_{2}\right) \boxplus \operatorname{Span}\left(\eta_{1}, \eta_{2}, \xi_{i} \eta_{j}, t \xi_{1}, t \xi_{2}\right) \\
& \cong(t+\Phi) \otimes \Lambda\left(\xi_{1}, \xi_{2}\right) \boxplus \mathfrak{g l}(2 \mid 1),
\end{aligned}
$$




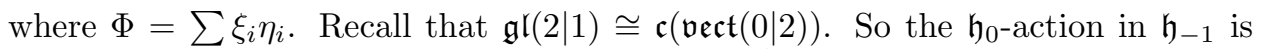
as follows: $(t+\Phi) \otimes \Lambda\left(\xi_{1}, \xi_{2}\right)$ commutes with $\mathbb{C}^{2 \mid 1}$ and acts by multiplication on $\Pi\left(\Lambda\left(\xi_{1}, \xi_{2}\right)\right)$ while $\mathfrak{g l}(2 \mid 1) \cong \mathfrak{c}(\mathfrak{v e c t}(0 \mid 2))$ acts as $T^{1 / 2} \otimes \chi_{0}$ in the vectorial realization in $\Pi\left(\Lambda\left(\xi_{1}, \xi_{2}\right)\right)$ and as $\operatorname{id}(\mathfrak{g l}(2 \mid 1))$ in $\mathbb{C}^{2 \mid 1}$. So $\mathfrak{h}_{-1}$, as $\mathfrak{h}_{0}$-module, is reducible and the regrading is not a $\mathrm{W}$ - regrading.

Cases $1 \xi+1 \eta$ and $2 \eta$ are similar to case $2 \xi$ with the same outcome; they are not $\mathrm{W}$ - regradings.

$6 \mathrm{c}) \mathfrak{g}=\mathfrak{k}(1 \mid 6 ; 3)$ induces four regradings of $\mathfrak{k} \mathfrak{a s}: 3 \xi, 2 \xi+1 \eta, 1 \xi+2 \eta$ and $3 \eta$. To describe them, consider the nonpositive terms of $\mathfrak{k}(1 \mid 6 ; 3)$ :

\begin{tabular}{|c|c|}
\hline-1 & 0 \\
\hline$\Lambda(3)$ & $\Lambda(3) \boxplus \mathfrak{v e c t}(0 \mid 3)$ \\
\hline
\end{tabular}

Consider two cases:

(i) $\mathfrak{h}=\mathfrak{k} \mathfrak{a} \mathfrak{s}(; 2 \xi+1 \eta)$ or $\mathfrak{k} \mathfrak{a} \mathfrak{s}(; 3 \xi)$. Then $\mathfrak{h}_{-1}=\Lambda(3), \mathfrak{h}_{0}=\Lambda(3) \boxplus \mathfrak{s l}(1 \mid 3)$; here $\mathfrak{s l}(1 \mid 3)$ is considered as the subalgebra of $\mathfrak{v e c t}(0 \mid 3)$.

(ii) $\mathfrak{h}=\mathfrak{k} \mathfrak{a s}(; 1 \xi+2 \eta)$ or $\mathfrak{k a s}(; 3 \eta)$. Then $\mathfrak{h}_{-1}=\operatorname{Vol}_{0}(0 \mid 3), \mathfrak{h}_{0}=\mathfrak{c}(\mathfrak{v e c t}(0 \mid 3))$.

Statement. All the W-regradings of $\mathfrak{k a s}$ are isomorphic to one of the following: $\mathfrak{k a s}(; 1), \mathfrak{k} \mathfrak{a} \mathfrak{s}(; 3 \xi)$ or $\mathfrak{k} \mathfrak{a s}(; 3 \eta)$.

Proof is similar to that of Statement 7.3.1.

7.7. W-regradings of $\mathfrak{g}=\mathfrak{k}_{\mathfrak{s l e}}(9 \mid 6)$. Let $\xi_{i}, q_{i}$ be the standard indeterminates of $\mathfrak{s l e}^{\circ}(4) ; \mathfrak{g}_{-2}=\mathbb{C} \cdot c$.

7 a) $\mathfrak{g}=\mathfrak{k} \mathfrak{s l e}(9 \mid 6 ; 1)$ is determined by the formulas

$$
\operatorname{deg} \xi_{1}=0 ; \operatorname{deg} q_{1}=2 ; \operatorname{deg} \xi_{i}=\operatorname{deg} q_{i}=1 \text { for } i>1 .
$$

Then $\mathfrak{h}_{-2}=\mathbb{C} \cdot \xi_{1}$ and $\mathfrak{h}_{-1}=\operatorname{Span}\left(\xi_{i}, \xi_{1} q_{i}, q_{i}, \xi_{1} \xi_{i}\right.$ for $\left.i>1 ; c,\left(\xi_{2} \xi_{3} \xi_{4}\right)^{*}\right)$, where $\left(\xi_{2} \xi_{3} \xi_{4}\right)^{*}$ is the dual to $\xi_{2} \xi_{3} \xi_{4} \in \mathfrak{g}_{1}$.

It is not difficult to see that the bracket determines a nondegenerate even skew

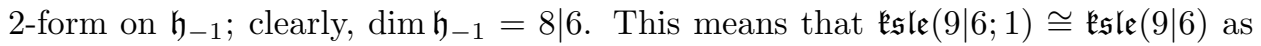
$\mathbb{Z}$-graded Lie superalgebras.

Denote this isomorphism by $R G$. Let $\mathfrak{h}=\tilde{c}\left(\mathfrak{s l e}^{\circ}(4 ; 4)\right) \subset \mathfrak{k s l e}_{\mathfrak{e}}(9 \mid 6)$; sec. 6.5. Then $R G(\mathfrak{h}) \subset \mathfrak{k s l e}_{\mathfrak{e}}(9 \mid 6)$ is isomorphic to $\mathfrak{h}$ as an abstract superalgebra, but not as $\mathbb{Z}$-graded one. We have

$$
\begin{aligned}
\mathfrak{h} \cap R G(\mathfrak{h}) & =\mathbb{C} \cdot c+\left(\mathfrak{s} \mathfrak{b}^{\circ}\left(\xi_{2}, \xi_{3}, \xi_{4}\right) \otimes \Lambda\left(\xi_{1}\right)\right) / \mathbb{C} \cdot 1 \boxplus \mathbb{C} \cdot \partial_{\xi_{1}} \\
& \left.\cong \tilde{\mathfrak{c}}\left(\mathfrak{s} \mathfrak{b}^{\circ}(3) \otimes \Lambda(1)\right) / \mathbb{C} \cdot 1\right) \boxplus \mathfrak{s v e c t}(1) .
\end{aligned}
$$

The regrading $R G$ determines a nontrivial automorphism of $\mathfrak{h} \cap R G(\mathfrak{h})$, generated by the automorphism $R$ of $\mathfrak{s l e}^{\circ}(3)$ (sec. 4.3) and permuting the central elements $c$ and $\xi_{1}$.

The nondirect sum of the linear spaces $\mathfrak{h}$ and $R G(\mathfrak{h})$ spans the whole of $\mathfrak{k} \mathfrak{s} \mathfrak{e}(9 \mid 6)$ :

$$
\mathfrak{h}+R G(\mathfrak{h})=\mathfrak{k}_{\mathfrak{s l e}}(9 \mid 6) .
$$

$7 \mathrm{~b}) \mathfrak{h}=\mathfrak{k}_{\mathfrak{s l e}}(9 \mid 6 ; 2)$ is determined by the formulas

$\operatorname{deg} \xi_{1}=\operatorname{deg} \xi_{2}=\operatorname{deg} q_{1}=\operatorname{deg} q_{2}=1 ; \operatorname{deg} \xi_{3}=\operatorname{deg} \xi_{4}=0 ; \quad \operatorname{deg} q_{3}=\operatorname{deg} q_{4}=2$.

Then $\mathfrak{h}_{-2}=\operatorname{Span}\left(c, \xi_{3}, \xi_{4}, \xi_{3} \xi_{4}\right)$ and $\operatorname{dim} \mathfrak{h}_{-2}=3 \mid 1$.

$$
\begin{aligned}
& \mathfrak{h}_{-1}=\operatorname{Span}\left(\xi_{1}, \xi_{2}, q_{1}, q_{2}\right) \otimes \Lambda\left(\xi_{3}, \xi_{4}\right) \cong V_{2} \otimes \Lambda(3) ; \\
& \mathfrak{h}_{0} \cong(\mathfrak{s l}(2) \otimes \Lambda(3)) \boxplus \mathfrak{s l}(3 \mid 1),
\end{aligned}
$$


where $\mathfrak{s l}(3 \mid 1) \subset \mathfrak{v e c t}(0 \mid 3)$. The $\mathfrak{h}_{0}$-action in $\mathfrak{h}_{-1}$ is the natural one.

$7 \mathrm{c}) \mathfrak{g}=\mathfrak{k} \mathfrak{s} \mathfrak{e}(9 \mid 6 ; K)$. Set

$$
\operatorname{deg} q_{i}=2 ; \quad \operatorname{deg} \xi_{i}=1 \text { for all } i .
$$

Then we obtain the compatible grading

$$
\mathfrak{h}_{-2}=\mathrm{id}, \mathfrak{h}_{-2}=\Lambda^{2}(\mathrm{id}), \mathfrak{h}_{0}=\mathfrak{s l}(5) .
$$

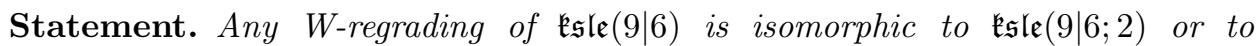
$\mathfrak{k}_{\mathfrak{S l e}}(9 \mid 6 ; K)$

Proof is similar to that of Statement 7.3.1.

Appendix 1. The solution of the system (2.1)-(2.4)

Let $D=\sum_{1 \leq i \leq 4}\left(P_{i} \frac{\partial}{\partial \xi_{i}}+Q_{i} \frac{\partial}{\partial u_{i}}\right) \in \mathfrak{g}^{\lambda}$ be an homogeneous (with respect to parity) vector field. Then by Lemma 2.1 it satisfies the system (2.1)-(2.4).

Equations (2.3) imply that there exists a function $f=f(u, \xi)$ such that $Q_{i}=$ $-(-1)^{p(D)} \frac{\partial f}{\partial \xi_{i}}$. Equations (2.1) imply further that

$$
P_{i}=\frac{\partial f}{\partial u_{i}}+\varphi_{i}\left(u ; \xi_{i}\right)=\frac{\partial f}{\partial u_{i}}+\varphi_{i}^{\circ}(u)+\varphi_{i}^{1}(u) \cdot \xi_{i}
$$

or, in other words, $D=L e_{f}+\sum\left(\varphi_{i}{ }^{\circ}(u)+\varphi_{i}^{1}(u) \xi_{i}\right) \frac{\partial}{\partial \xi_{i}}$. Equations (2.2) now imply that

$$
\varphi_{i}^{1}(u)=\frac{\partial \varphi_{i}}{\partial \xi_{i}}=\frac{1}{2}(-1)^{p(D)} \sum \frac{\partial Q_{j}}{\partial u_{j}}=-\frac{1}{2} \Delta(f), \text { where } \Delta=\sum \frac{\partial^{2}}{\partial u_{i} \partial \xi_{i}}
$$

whereas equations (2.4) imply that

$$
\frac{\partial \varphi_{i}}{\partial u_{j}}-\frac{\partial \varphi_{j}}{\partial u_{i}}=-2 \lambda \frac{\partial^{2} f}{\partial \xi_{l} \partial \xi_{k}} .
$$

Remark. Let $\psi_{i}=\psi_{i}(u)$, where $i=1, \ldots, 4$, be a set of functions such that $\frac{\partial \psi_{i}}{\partial u_{j}}-$ $\frac{\partial \psi_{j}}{\partial u_{i}}=0$. Then there exists a function $\psi(u)$ for which $\psi_{i}=\frac{\partial \psi}{\partial u_{i}}$ and $\sum \psi_{i}(u) \frac{\partial}{\partial \xi_{i}}=$ $L e_{\psi}=D_{\psi}$ (see heading 2) from the corollary in Theorem 2.3). Thus, for any function $f$ it suffices to find any collection of functions $\varphi_{i}{ }^{\circ}$.

With the help of the differential forms

$$
\alpha=\sum_{i \leq 4} \varphi_{i} d u_{i} \text { and } \omega_{0}(f)=\sum_{(i, j, k, l) \in A_{4}} \frac{\partial^{2} f}{\partial \xi_{l} \partial \xi_{k}} d u_{j} \wedge d u_{i}
$$

equations $\left(2.2^{\prime}\right)$ and $\left(2.4^{\prime}\right)$ can be expressed in the form

$$
d \alpha=\frac{1}{2} \Delta(f) \cdot \omega-2 \lambda \cdot \omega_{0}(f) .
$$

Equation (A.1) is solvable if and only if the form in the right hand side is exact or, since our considerations are local, equivalently, if and only if it is closed.

Direct calculations show that the condition that the form in the right hand side is closed is equivalent to the system

$$
\left\{\begin{array}{l}
\frac{\partial f}{\partial \xi_{i}}=0 \quad \text { for all } i=1,2,3,4 \\
\frac{1}{2} \frac{\partial \Delta f}{\partial u_{i}}-2 \lambda \frac{\partial^{3} f}{\partial \xi_{j} \partial \xi_{k} \partial \xi_{l}}=0 \quad \text { for all } i=1,2,3,4 \text { and }(i, j, k, l) \in A_{4}
\end{array}\right.
$$


Equations (A.1.1) imply that $\Delta(f)$ only depends on $u$; equations (A.1.2) imply that $\operatorname{deg}_{\xi} f \leq 3$.

First, suppose that $\operatorname{deg}_{\xi} f \leq 2$. Then equations (A.1.2) imply that

$$
\Delta(f)=\text { const. }
$$

Denote $-\frac{1}{4} \Delta(f)$ by $c$. Thus, $f=-c \sum_{i<4} u_{i} \xi_{i}+f_{0}$, where $\Delta\left(f_{0}\right)=0$. By $\left(2.2^{\prime}\right)$ then, $\varphi_{i}^{1}(u)=2 c$ and $D=L e_{-c \sum u_{i} \xi_{i}+f_{0}}+\sum \varphi_{i}{ }^{\circ} \frac{\partial}{\partial \xi_{i}}+2 c \sum \xi_{i} \frac{\partial}{\partial \xi_{i}}=L e_{f_{0}}+$ $\sum \varphi_{i}{ }^{\circ} \frac{\partial}{\partial \xi_{i}}+c Z$. Replace $f$ with $f_{0}$. Then $\Delta(f)=0$ and we have

$$
\frac{\partial \varphi_{i}^{\circ}}{\partial u_{j}}-\frac{\partial \varphi_{j}^{\circ}}{\partial u_{i}}=-2 \lambda \frac{\partial^{2} f}{\partial \xi_{l} \partial \xi_{k}} .
$$

If $\operatorname{deg}_{\xi} f<2$, then the right hand side of $\left(2.4^{\prime \prime}\right)$ is equal to 0 . So due to Remark we can take $\varphi_{i}{ }^{\circ}=0$. In this case

$$
D=L e_{f}+c Z=D_{f}+c Z .
$$

Let $\operatorname{deg}_{\xi} f=2$. As $\Delta(f)=0$, we can set $g=\Delta^{-1}(f)$. Then $\operatorname{deg}_{\xi} g=3$ and $f=\Delta(g)$. For the role of functions $\varphi_{i}{ }^{\circ}$ satisfying equation $\left(2.4^{\prime \prime}\right)$ we can take

$$
\varphi_{i}^{\circ}=2 \lambda \frac{\partial^{3} g}{\partial \xi_{j} \partial \xi_{k} \partial \xi_{l}}, \text { where }(i, j, k, l) \in A_{4} .
$$

We get

$$
D_{f}=\operatorname{Le}_{f}+2 \lambda \sum_{(i, j, k, l) \in A_{4} ; 1 \leq i \leq 4} \frac{\partial^{3} g}{\partial \xi_{j} \partial \xi_{k} \partial \xi_{l}} \cdot \frac{\partial}{\partial \xi_{i}}+c Z=D_{f}+c Z .
$$

Let $\operatorname{deg}_{\xi} f=3$. Let us represent $f$ in the form $f=f_{3}+f_{<3}$, where $f_{3}$ is a homogeneous (with respect to the degree in $\xi$ ) polynomial of degree 3 , while $f_{<3}$ is a polynomial of lesser degree.

Since $\Delta(f)$ only depends on $u$, we see that $\Delta\left(f_{3}\right)=0$ and, therefore, we can introduce $H=\Delta^{-1}\left(f_{3}\right)=F(u) \xi_{1} \xi_{2} \xi_{3} \xi_{4}$ for some function $F(u)$.

From $\left(2.4^{\prime \prime}\right)$ we deduce that

$$
\frac{\partial \varphi_{i}^{1}}{\partial u_{j}}=2 \lambda \frac{\partial F}{\partial u_{j}} \text { or, with }\left(2.2^{\prime}\right), \varphi_{i}^{1}=2 \lambda F=-\frac{1}{2} \Delta(f) .
$$

Therefore, $\Delta(f)=\Delta\left(f_{<3}\right)=-4 \lambda F$. Set

$$
\hat{f}=4 \Delta^{-1}(F) .
$$

We see that $f=\Delta(H)-\lambda \hat{f}+g$ for some function $g$ such that $\Delta(g)=0$ and $\operatorname{deg}_{\xi} g<3$. But we have already described the solutions for all such $g$. So now we assume $g=0$. In this case we can take $\varphi_{i}{ }^{\circ}=0$ (due to Remark). We get

$$
\begin{aligned}
D & =\operatorname{Le}_{f}+\lambda\left(-\operatorname{Le}_{\hat{f}}+2 F \sum \xi_{i} \frac{\partial}{\partial \xi_{i}}\right) \\
& =\operatorname{Le}_{f}+\lambda\left(-\operatorname{Le}_{\hat{f}}+2 \sum_{(i, j, k, l) \in A_{4} ; 1 \leq i \leq 4} \frac{\partial^{3} H}{\partial \xi_{j} \partial \xi_{k} \partial \xi_{l}} \cdot \frac{\partial}{\partial \xi_{i}}\right)=D_{f} .
\end{aligned}
$$

Formulas (A.3)-(A.5) prove Theorem 2.3. 
Appendix 2. Proof of headings 2 of Theorems 4.2, 6.2 And 6.5

A.2.1. Lemma. Let $\left(\mathfrak{g}_{-1}, \mathfrak{g}_{0}\right)_{*}$ be simple; let the $\mathfrak{g}_{0}$-module $\mathfrak{g}_{-1}$ be irreducible. If $\mathfrak{h}=\left(\mathfrak{g}_{-1}, \mathfrak{c g}_{0}\right)_{*}$ is also simple, then for every $v \in \mathfrak{g}_{-1}$ there exists an $F \in \mathfrak{g}_{1}$ such that $[v, F] \notin \mathfrak{g}_{0}$.

The same applies to $\left(\mathfrak{g}_{-}, \mathfrak{g}_{0}\right)_{*}^{m k}$ and $\mathfrak{h}=\left(\mathfrak{g}_{-}, \mathfrak{c g}_{0}\right)_{*}^{m k}$.

Proof. By simplicity of $\left(\mathfrak{c g}_{0}\right)_{*}$ (due to Lemma 4.1) we have $\left[\mathfrak{h}_{-1}, \mathfrak{h}_{1}\right]=c \mathfrak{g}_{0}$, i.e., there exists $v_{0} \in \mathfrak{g}_{-1}, F_{0} \in \mathfrak{g}_{1}$ such that $\left[v_{0}, F_{0}\right] \notin \mathfrak{g}_{0}$.

Let

$$
V_{1}=\left\{v_{1} \in \mathfrak{g}_{-1}:\left[g, v_{1}\right]=v_{0} \text { for some } g \in \mathfrak{g}_{0}\right\} .
$$

Then for any $v_{1} \in V_{1}$ we have

$$
\mathfrak{g}_{0} \not \supset\left[\left[g, v_{1}\right], F_{0}\right]= \pm\left[\left[g, F_{0}\right], v_{1}\right] \pm\left[g,\left[v_{1}, F_{0}\right]\right],
$$

where the signs are governed by Sign Rule. Therefore, one of the two cases holds:

1) $\left[v_{1}, F_{0}\right] \notin \mathfrak{g}_{0}$, hence, $F=F_{0}$;

2) $\left[v_{1}, F_{0}\right] \in \mathfrak{g}_{0}$.

In case 2$)$ we have $\left[g,\left[v_{1}, F_{0}\right]\right] \in \mathfrak{g}_{0}$; hence,

$$
\left[F, v_{1}\right] \notin \mathfrak{g}_{0} \text { for } F=\left[g, F_{0}\right] .
$$

Similarly, introduce the sequence of spaces

$$
V_{2}=\left\{v_{2} \in \mathfrak{g}_{-1}:\left[g, v_{2}\right] \in V_{1}+<\operatorname{Span}\left(v_{0}\right) \text { for some } g \in \mathfrak{g}_{0}\right\}, \text { etc. }
$$

By irreducibility of $\mathfrak{g}_{0}$-action on $\mathfrak{g}_{-1}$ for every $v \in \mathfrak{g}_{-1}$ there exists an $n$ such that $v \in V_{n}$ and, therefore, $F=F_{n}$, where $\left[v, F_{n}\right] \notin \mathfrak{g}_{0}$.

The arguments for depth 2 are literally the same.

A.2.2. Corollary. Let $\mathfrak{g}_{-1}=\Pi(\Lambda(n) / \mathbb{C} 1), \mathfrak{g}_{0}=\mathfrak{v e c t}(0 \mid n)$, i.e., $\mathfrak{g}_{*}=\left(\mathfrak{g}_{-1}, \mathfrak{g}_{0}\right)_{*}=$ $\mathfrak{l} \mathfrak{e}(n ; n)$. Then $\left(\mathfrak{g}_{-1}, \mathfrak{c}\left(\mathfrak{g}_{0}\right)\right)_{*}$ is not simple for $n>3$.

Hereafter in this appendix we often abuse the notations and denote the elements by their generating functions.

Proof. By Lemma A.2.1 the simplicity of $\mathfrak{g}_{*}$ implies that for any $v \in \mathfrak{g}_{-1}$ there exists $F \in \mathfrak{g}_{-1}$ such that $[v, F] \notin \mathfrak{g}_{0}$.

Take $v=\xi_{1} \ldots \xi_{n}$; let $d$ be the central element of $\mathfrak{c}\left(\mathfrak{g}_{0}\right)$ normalized so that ad $\left.d\right|_{\mathfrak{g}_{-1}}=-$ id. Let $F \in \mathfrak{g}_{1}$ be such that

$$
[v, F]=d+g, \text { for } g \in \mathfrak{g}_{0} .
$$

Then

$$
\pm\left[v,\left[F, v_{1}\right]\right] \stackrel{\text { by Jacobi id. }}{=}\left[[v, F], v_{1}\right]=(d+g) v_{1}=-v_{1}+g v_{1} .
$$

In other words, $g_{1}=\left[F, v_{1}\right]$ maps $v$ to $-v_{1}+g v_{1}$ up to a sign. But in the $\mathfrak{g}_{0}$-module considered, the image of $v$ can only be a function of degree $\geq n-1$.

Hence, $g v_{1}=v_{1}+\varphi\left(v_{1}\right)$, where $\operatorname{deg} \varphi \geq n-1$, for any $v_{1}$ of degree $<n-1$. Consequently, the projection $g_{0}$ of $g$ on the zeroth component of $\mathfrak{v e c t}(0 \mid n)$ with respect to the standard $\mathbb{Z}$-grading, i.e., on $\mathfrak{g l}(n)$, satisfies the condition

$$
\left.g_{0}\right|_{\operatorname{Span}\left(v_{1}: \operatorname{deg} v_{1}<n-1\right)}=\mathrm{id} .
$$

But in $\mathfrak{v e c t}(0 \mid n)$ the dimension of the maximal torus $\operatorname{Span}\left(\varepsilon_{i} \partial_{i}: 1 \leq i \leq n\right)$ is equal to $n$ and there is no operator whose restrictions to the spaces of homogenuous functions in $\xi$ of at least two distinct degrees are scalar operators. 
Since $n-2 \geq 2$ for $n>3$, the Lie superalgebra $\left(\mathfrak{g}_{-1}, \mathfrak{c g}_{0}\right)_{*}$ is not simple.

A.2.3. Corollary. Let

$$
\mathfrak{g}_{-}=\left\{\begin{array}{cc}
\mathfrak{a} \mathfrak{b}\left(2^{n-1}\right) & \text { for } n \text { odd }, \\
\mathfrak{h} \mathfrak{i}\left(2^{n-1} \mid 2^{n-1}\right) & \text { for } n \text { even } .
\end{array}\right.
$$

The Lie superalgebra $\left(\mathfrak{g}_{-}, \mathfrak{c} \mathfrak{b e c t}(0 \mid n)\right)_{*}^{m k}$ is not simple for $n>3$.

Proof follows the lines of the proof of Corollary A.2.1 with the correction that (A.2.0) is now true not for all $v_{1} \in \mathfrak{g}_{-1}$ but only for those which satisfy $\left[v_{1}, v\right]=0$. Such elements $v_{1}$ are represented by functions $f \in \Lambda(n)$ such that $0<\operatorname{deg} f \leq n-1$. There are $\geq 2$ distinct degrees which satisfy this inequality for $n>3$.

A.2.4. Corollary. Let

$$
\mathfrak{g}_{-}=\left\{\begin{array}{cc}
\mathfrak{a} \mathfrak{b}\left(2^{n-1}-1\right) & \text { for } n \text { odd } \\
\mathfrak{h e i}\left(2^{n-1} \mid 2^{n-1}-2\right) & \text { for } n \text { even }
\end{array}\right.
$$

Then $\mathfrak{g}=\left(\mathfrak{g}_{-}, \mathfrak{s v e c t}_{a, b}(0 \mid n)\right)_{*}^{m k}$ is not a simple Lie superalgebra if either $n>4$ or $n=4$ and $(a, b) \notin \mathbb{C}(3,4)$.

Proof is obtained by a slight modification of the proof of Corollary A.2.2. As $v$ we now take $\xi_{1} \ldots \xi_{n-1} \in \mathfrak{g}_{-1}$; let $F$ be such that $[v, F]=a x+b d+g$, where $g \in \mathfrak{s v e c t}(0 \mid n)$. Then

$$
\left[\left[F, v_{1}\right], v\right]= \pm\left[[v, F], v_{1}\right]=(a k-b) v_{1}+g v_{1}
$$

for any monomial $v_{1} \in \mathfrak{g}_{-1}$ of degree $k$ and distinct from $\xi_{n}$. Since every element from $\mathfrak{g}_{0}$ lowers the degree of any monomial not more than by 1 , we see that the projection $g_{0}$ of $g$ on $\mathfrak{s w e c t}(0 \mid n)_{0}$ satisfies the relation

$$
g_{0} v_{1}=(b-a k) v_{1}
$$

for any monomial $v_{1} \in \mathfrak{g}_{-1}$ of degree $k<n-2$ and distinct from $\xi_{n}$. In particular, for $n>4$ this means that $g_{0}$ acts on $\operatorname{Span}\left(\xi_{1}, \ldots, \xi_{n-1}\right)$ by multiplication by $b-a$ and on $\Lambda^{2}(\xi)$ by multiplication by $b-2 a$. Hence, $g_{0}=0$, i.e., $a=b=0$.

In A.2.2 $k<n-2$. So if $n=4$, then $k=1$. The component $g_{0}$ is defined by its action on $\xi_{1}, \ldots, \xi_{n}$. But A.2.2 gives the action of $g_{0}$ only on $\xi_{1}, \ldots, \xi_{n-1}$. Its action on $\xi_{n}$ can be arbitrary with only one condition: $g_{0} \in \mathfrak{s v e c t}(0 \mid 4)$; this is what A.2.3 means:

$$
g_{0}\left(\xi_{1} \xi_{4}\right)=-2(b-a) \xi_{1} \xi_{4}+c_{1} \xi_{1} \xi_{2}+c_{2} \xi_{1} \xi_{3} .
$$

Look at formula (A.2.1) with $v=\xi_{1} \xi_{2} \xi_{3}$ and $v_{1}=\xi_{1} \xi_{4}$. It means that $a d\left[F, v_{1}\right]$ (which is an element from $\mathfrak{s v e c t}(0 \mid 4) \boxplus \mathbb{C}(a x+b d))$ sends $\xi_{1} \xi_{2} \xi_{3}$ to $(2 a-b) \xi_{1} \xi_{4}+$ $g\left(\xi_{1} \xi_{4}\right)$. Since no vector field can send $v$ to $v_{1}$, we deduce that $g_{0}\left(v_{1}\right)$ must compensate $(2 a-b) \xi_{1} \xi_{4}$. But from formula (A.2.3) we derive that $b-2 a=-2 b+2 a$, implying $3 b=4 a$.

Due to Lemmas 4.1, 6.1, Corollaries A.2.2-A.2.4 are equivalent to the headings 2) of Theorems 4.2, 6.2 and 6.5 , respectively.

Appendix 3. Proof of Simplicity of the Lie superalgebras $\mathfrak{m b}(4 \mid 5)=(\mathfrak{a b}(4), \mathfrak{c} \mathfrak{v e c t}(0 \mid 3))_{*}^{m}$ AND $\mathfrak{k s t e}_{\mathfrak{c}}(9 \mid 6)=\left(\mathfrak{h} \mathfrak{e i}(8 \mid 6), \mathfrak{s v e c t}(4)_{3,4}\right)_{*}^{k}$

In this appendix $\mathfrak{g}$ is either $\mathfrak{m} \mathfrak{b}(4 \mid 5)$ or $\mathfrak{k} \mathfrak{s} \mathfrak{e}(9 \mid 6)$. Due to Lemma 6.1, to prove the simplicity of $\mathfrak{g}$ it suffices to exibit an element $\hat{F} \in \mathfrak{g}_{1}$ such that

(A.3.1) $\left[\mathfrak{g}_{-1}, \hat{F}\right]$ is not entirely contained in $\mathfrak{v e c t}(0 \mid 3)$ and $\mathfrak{s v e c t}(0 \mid 3)$, respectively. 
A.3.1. Simplicity of $(\mathfrak{a} \mathfrak{b}(4), \mathfrak{c v e c t}(0 \mid 3))_{*}^{m}$. First, let us show how to embed $\mathfrak{g}=$ $\mathfrak{c} \mathfrak{v e c t}(0 \mid 3)_{*}^{m}$ into $\mathfrak{m}(4)$. We consider $\mathfrak{m}(4)$ as preserving the Pfaff equation given by the form $\alpha_{0}=d \tau+\sum_{i=0}^{3}\left(\eta_{i} d u_{i}+u_{i} d \eta_{i}\right)$. Denote the basis elements of $\mathfrak{g}$ as follows:

\begin{tabular}{|c|c|c|}
\hline $\mathfrak{g}_{-2}$ & A basis of $\mathfrak{g}_{-1}=\Pi\left(\operatorname{Vol}^{\frac{1}{2}}\right)$ & $\begin{array}{r}\text { notations of the corres. functions } \\
\text { that generate } \mathfrak{g}_{-1} \subset \mathfrak{m}(4)\end{array}$ \\
\hline \multirow{3}{*}{$M_{1}$} & $\xi_{1} \xi_{2} \xi_{3}$ & $\eta_{0}$ \\
& $\xi_{2} \xi_{3}, \quad \xi_{3} \xi_{1}, \quad \xi_{1} \xi_{2}$ & $u_{1}, \quad u_{2}, u_{3}$ \\
& $\xi_{1}, \quad \xi_{2}, \quad \xi_{3}$ & $\eta_{1}, \quad \eta_{2}, \eta_{3}$ \\
& 1 & $u_{0}$ \\
\hline
\end{tabular}

The following is an explicit realization of the embedding $i: \mathfrak{g}_{0}=\operatorname{vect}(0 \mid 3) \longrightarrow$ $\mathfrak{l}_{\mathfrak{e}}(3)$. We only indicate the generating functions of the image:

\begin{tabular}{|c|c|}
\hline $\operatorname{deg} D$ & $D \in \mathfrak{v e c t}(0 \mid 3)$ \\
\hline-1 & $\partial_{1}, \quad \partial_{2}, \quad \partial_{3}$ \\
\hline 0 & $\xi_{i} \partial_{j}$ for $i \neq j$ \\
\hline 0 & $\xi_{1} \partial_{1}, \quad \xi_{2} \partial_{2}, \quad \xi_{3} \partial_{3}$ \\
\hline 1 & $\xi_{2} \xi_{3} \partial_{1}, \quad \xi_{3} \xi_{1} \partial_{2}, \quad \xi_{1} \xi_{2} \partial_{3}$ \\
\hline 1 & $\xi_{1}\left(\xi_{2} \partial_{2}-\xi_{3} \partial_{3}\right), \quad \xi_{2}\left(\xi_{3} \partial_{3}-\xi_{1} \partial_{1}\right), \quad \xi_{3}\left(\xi_{1} \partial_{1}-\xi_{2} \partial_{2}\right)$ \\
\hline 1 & $\xi_{1}\left(\xi_{2} \partial_{2}+\xi_{3} \partial_{3}\right), \quad \xi_{2}\left(\xi_{3} \partial_{3}+\xi_{1} \partial_{1}\right), \quad \xi_{3}\left(\xi_{1} \partial_{1}+\xi_{2} \partial_{2}\right)$ \\
\hline 2 & $\xi_{1} \xi_{2} \xi_{3} \partial_{i}$ \\
\hline
\end{tabular}

The respective images $i(D)$ are as follows:

\begin{tabular}{|c|}
\hline$-u_{0} u_{1}+\eta_{2} \eta_{3}, \quad-u_{0} u_{2}+\eta_{3} \eta_{1}, \quad-u_{0} u_{3}+\eta_{1} \eta_{2}$ \\
\hline$-u_{i} \eta_{j}$ for $i \neq j ; i, j>0$ \\
$\frac{1}{2}\left(-u_{0} \eta_{0}-u_{1} \eta_{1}+u_{2} \eta_{2}+u_{3} \eta_{3}\right)$ \\
$\frac{1}{2}\left(-u_{0} \eta_{0}+u_{1} \eta_{1}-u_{2} \eta_{2}+u_{3} \eta_{3}\right)$ \\
$\frac{1}{2}\left(-u_{0} \eta_{0}+u_{1} \eta_{1}+u_{2} \eta_{2}-u_{3} \eta_{3}\right)$ \\
\hline$-\frac{1}{2} u_{1}^{2}, \quad-\frac{1}{2} u_{2}^{2}, \quad-\frac{1}{2} u_{3}^{2}$ \\
$-u_{2} u_{3}, \quad-u_{1} u_{3}, \quad-u_{1} u_{2}$ \\
$\eta_{0} \eta_{1}, \quad \eta_{0} \eta_{2}, \quad \eta_{0} \eta_{3}$ \\
\hline
\end{tabular}

To check condition (A.3.1), take

$$
\hat{F}=M_{F} \text {, where } F=2 \tau u_{1}-2 \eta_{0} \eta_{2} \eta_{3}+u_{0}^{2} \eta_{1} \text {. }
$$

Then the brackets with $\mathfrak{g}_{-1}$ are

$$
\begin{array}{ll}
\left\{F, u_{0}\right\}_{m . b .}=-2 u_{0} u_{1}+2 \eta_{2} \eta_{3} ; & \left\{F, \eta_{1}\right\}_{m . b .}=2 \tau ; \\
\left\{F, u_{1}\right\}_{m . b .}=-3 u_{1}^{2} ; & \left\{F, \eta_{i}\right\}_{m . b .}=-2 u_{1} \eta_{i}(i=0,2,3) ; \\
\left\{F, u_{2}\right\}_{m . b .}=-2 u_{1} u_{2}-2 \eta_{0} \eta_{3} ; & \left\{F, u_{3}\right\}_{m . b .}=-2 u_{1} u_{3}+2 \eta_{0} \eta_{2} .
\end{array}
$$


We get $M_{\tau}$, while the remaining elements in the right hand sides of (A.3.2) lie in $\mathfrak{v e c t}(0 \mid 3)$.

A.3.2. Simplicity of $\left(\mathfrak{h e i}(8 \mid 6), \mathfrak{s v e c t}(0 \mid 4)_{3,4}\right)_{*}^{k}$. We first embed $(\mathfrak{h e i}(8 \mid 6), \mathfrak{s v e c t}(0 \mid 4))_{*}^{k}$ into $\mathfrak{k}(9 \mid 6)$. We realize $\mathfrak{k}(9 \mid 6)$ as preserving the Pfaff equation given by the form

$$
\alpha_{1}=d t-\sum_{i \leq 4}\left(p_{i} d q_{i}-d q_{i} p_{i}\right)-\sum_{j \leq 3}\left(\eta_{j} d \xi_{j}+\xi_{j} d \eta_{j}\right)
$$

Let us redenote the basis elements of $\mathfrak{g}_{-1}$ :

\begin{tabular}{|c|c|}
\hline $\begin{array}{c}\text { A basis of } \mathfrak{g}_{-1} \\
=\Pi\left(T_{0}^{0}(\overrightarrow{0})\right)\end{array}$ & $\begin{array}{c}\text { notations of the corresp. functions } \\
\text { that generate } \mathfrak{g}_{-1} \subset \mathfrak{k}(9 \mid 6)\end{array}$ \\
\hline$\xi_{1}, \quad \xi_{2}, \quad \xi_{3}, \quad \xi_{4}$ & $p_{1}, \quad p_{2}, \quad p_{3}, \quad p_{4}$ \\
$\xi_{1} \xi_{2}, \quad \xi_{1} \xi_{3}, \quad \xi_{1} \xi_{4}$ & $\eta_{1}, \quad \eta_{2}, \quad \eta_{3}$ \\
$-\xi_{3} \xi_{4}, \quad \xi_{2} \xi_{4}, \quad-\xi_{2} \xi_{3}$ & $\zeta_{1}, \quad \zeta_{2}, \quad \zeta_{3}$ \\
$\xi_{2} \xi_{3} \xi_{4}, \quad-\xi_{1} \xi_{3} \xi_{4}, \quad \xi_{1} \xi_{2} \xi_{4}, \quad-\xi_{1} \xi_{2} \xi_{3}$ & $q_{1}, \quad q_{2}, \quad q_{3}, \quad q_{4}$ \\
\hline
\end{tabular}

The following is an explicit realization of the embedding $i: \mathfrak{g}_{0}=\mathfrak{s v e c t}(0 \mid 4) \longrightarrow$ $\mathfrak{h}(8 \mid 6)_{0}$. We only indicate the generating functions of the image. For $D \in \mathfrak{s v e c t}(0 \mid 4)$ we have

$\underline{\operatorname{deg} D=-1}$ :

$$
\begin{aligned}
\partial_{\xi_{1}} & \mapsto \zeta_{1} p_{2}+\zeta_{2} p_{3}+\zeta_{3} p_{4} \\
\partial_{\xi_{2}} & \mapsto-\zeta_{1} p_{1}+\eta_{2} p_{4}-\eta_{3} p_{3} \\
\partial_{\xi_{3}} & \mapsto-\zeta_{2} p_{1}+\eta_{3} p_{2}-\eta_{1} p_{4} \\
\partial_{\xi_{4}} & \mapsto-\zeta_{3} p_{1}-\eta_{2} p_{2}+\eta_{1} p_{3}
\end{aligned}
$$

$\operatorname{deg} D=0$ :

$$
\begin{array}{rlrl}
\xi_{1} \partial_{2} & \mapsto-p_{1} q_{2}-\eta_{2} \eta_{3} & \xi_{2} \partial_{3} & \mapsto-p_{2} q_{3}+\eta_{1} \zeta_{2} \\
\xi_{2} \partial_{1} & \mapsto-p_{2} q_{1}+\zeta_{2} \zeta_{3} & \xi_{3} \partial_{2} & \mapsto-p_{3} q_{2}+\eta_{2} \zeta_{1} \\
\xi_{1} \partial_{3} & \mapsto-p_{1} q_{3}+\eta_{1} \eta_{3} & \xi_{2} \partial_{4} & \mapsto-p_{2} q_{4}+\eta_{1} \zeta_{3} \\
\xi_{3} \partial_{1} & \mapsto-p_{3} q_{1}-\zeta_{1} \zeta_{3} & \xi_{4} \partial_{2} & \mapsto-p_{4} q_{2}+\eta_{3} \zeta_{1} \\
\xi_{1} \partial_{4} & \mapsto-p_{1} q_{4}-\eta_{1} \eta_{2} & \xi_{3} \partial_{4} & \mapsto-p_{3} q_{4}+\eta_{2} \zeta_{3} \\
\xi_{4} \partial_{1} & \mapsto-p_{4} q_{1}+\zeta_{1} \zeta_{2} & \xi_{4} \partial_{3} & \mapsto-p_{4} q_{3}+\eta_{3} \zeta_{2} \\
\xi_{1} \partial_{1}-\xi_{2} \partial_{2} & \mapsto & -p_{1} q_{1}+p_{2} q_{2}+\eta_{2} \zeta_{2}+\eta_{3} \zeta_{3} \\
\xi_{2} \partial_{2}-\xi_{3} \partial_{3} & \mapsto & -p_{2} q_{2}+p_{3} q_{3}+\eta_{1} \zeta_{1}-\eta_{2} \zeta_{2} \\
\xi_{3} \partial_{3}-\xi_{4} \partial_{4} & \mapsto & -p_{3} q_{3}+p_{4} q_{4}+\eta_{2} \zeta_{2}-\eta_{3} \zeta_{3} \\
\sum \xi_{i} \partial_{i} & \mapsto & -\sum p_{i} q_{i}-2 t
\end{array}
$$

$\operatorname{deg} D=1$ :

$$
\begin{array}{rlrl}
\xi_{1} \xi_{2} \partial_{3} & \mapsto-\eta_{1} q_{3}, \xi_{2} \xi_{3} \partial_{1} \mapsto-\eta_{3} q_{1}, \xi_{3} \xi_{1} \partial_{2} \mapsto-\eta_{2} q_{2} \\
\xi_{1} \xi_{2} \partial_{1}+\xi_{2} \xi_{3} \partial_{3} & \mapsto-q_{1} \eta_{1}+q_{3} \zeta_{3} & \xi_{1} \xi_{4} \partial_{1}+\xi_{4} \xi_{2} \partial_{2} & \mapsto-q_{1} \eta_{3}+q_{2} \zeta_{2} \\
\xi_{1} \xi_{2} \partial_{1}+\xi_{2} \xi_{4} \partial_{4} & \mapsto-q_{1} \eta_{1}-q_{4} \zeta_{2} & \xi_{1} \xi_{4} \partial_{1}+\xi_{4} \xi_{3} \partial_{3} & \mapsto-q_{1} \eta_{3}-q_{3} \zeta_{1} \\
\xi_{1} \xi_{3} \partial_{1}+\xi_{3} \xi_{2} \partial_{2} & \mapsto-q_{1} \eta_{2}-q_{2} \zeta_{3} & \xi_{1} \xi_{2} \partial_{2}-\xi_{1} \xi_{3} \partial_{3} & \mapsto-q_{2} \eta_{1}+q_{3} \eta_{2} \\
\xi_{1} \xi_{3} \partial_{1}+\xi_{3} \xi_{4} \partial_{4} & \mapsto-q_{1} \eta_{2}+q_{4} \zeta_{1} & \xi_{1} \xi_{2} \partial_{2}-\xi_{1} \xi_{4} \partial_{4} & \mapsto-q_{2} \eta_{1}+q_{4} \eta_{3}
\end{array}
$$

$\operatorname{deg} D=2$ : The image under $i$ is generated by $q_{i} q_{j}$ for any $1 \leq i, j \leq 4$; it is inessential to us since $\mathfrak{s v e c t}(0 \mid 4)_{2}$ is generated by $\mathfrak{s v e c t}(0 \mid 4)_{1}$. 
Now, set

$$
\begin{array}{ll}
x_{0}=K_{-\sum p_{i} q_{i}-2 t,} & x_{1}=K_{-p_{1} q_{1}+p_{2} q_{2}+\eta_{2} \zeta_{2}+\eta_{3} \zeta_{3}}, \\
x_{2}=K_{-p_{2} q_{2}+p_{3} q_{3}+\eta_{1} \zeta_{1}-\eta_{2} \zeta_{2},}, & x_{3}=K_{-p_{3} q_{3}+p_{4} q_{4}+\eta_{2} \zeta_{2}-\eta_{3} \zeta_{3}} ;
\end{array}
$$

see (A.3.3). Set

$$
f=t+\sum_{i \leq 3} p_{i} q_{i}+3 p_{4} q_{4}+\eta_{1} \zeta_{1}+\eta_{2} \zeta_{2}-\eta_{3} \zeta_{3}
$$

Then

$$
K_{f}=\frac{1}{2} x_{1}+x_{2}+\frac{3}{2} x_{3}-\frac{3}{2} x_{0}-2 K_{t} \in \mathfrak{s v e c t}(0 \mid 4) \boxplus \mathbb{C}\left(3 x_{0}+4 K_{t}\right) .
$$

To check the condition (A.3), take $\hat{F}=K_{F}$, where

$$
F=t p_{4}+p_{4}\left(\sum_{i \leq 4} p_{i} q_{i}+\eta_{1} \zeta_{1}+\eta_{2} \zeta_{2}-\eta_{3} \zeta_{3}\right)-2 \zeta_{1} \zeta_{2} p_{1}+2 \zeta_{1} \eta_{3} p_{2}+2 \zeta_{2} \eta_{3} p_{3} .
$$

The commutators of $F$ with $\mathfrak{k}_{-1}(9 \mid 6)$ are of the form:

$$
\begin{array}{ll}
\left\{q_{i}, F\right\}_{k . b .}=q_{i} \frac{\partial F}{\partial t}+\frac{\partial F}{\partial p_{i}} ; & \left\{\eta_{i}, F\right\}_{k . b .}=\eta_{i} \frac{\partial F}{\partial t}-\frac{\partial F}{\partial \zeta_{i}} \\
\left\{p_{i}, F\right\}_{k . b .}=p_{i} \frac{\partial F}{\partial t}-\frac{\partial F}{\partial q_{i}} ; & \left\{\zeta_{i}, F\right\}_{k . b .}=\zeta_{i} \frac{\partial F}{\partial t}-\frac{\partial F}{\partial \eta_{i}} .
\end{array}
$$

Hence,

$$
\begin{aligned}
& \left\{q_{4}, F\right\}_{k . b .}=f ; \\
& \left\{\eta_{1}, F\right\}_{k . b .}=2\left(\eta_{1} p_{4}+\zeta_{2} p_{1}-\eta_{3} p_{2}\right) \mapsto-2 \partial_{3} ; \\
& \left\{\eta_{2}, F\right\}_{k . b .}=2\left(\eta_{2} p_{4}-\zeta_{1} p_{1}-\eta_{3} p_{3}\right) \mapsto 2 \partial_{2} ; \\
& \left\{\eta_{3}, F\right\}_{k . b .}=\left\{\zeta_{1}, F\right\}_{k . b .}=\left\{\zeta_{2}, F\right\}_{k . b .}=0 ; \\
& \left\{\zeta_{3}, F\right\}_{k . b .}=2\left(\zeta_{3} p_{4}+\zeta_{1} p_{2}+\zeta_{2} p_{3}\right) \mapsto 2 \partial_{1} ; \\
& \left\{q_{1}, F\right\}_{k . b .}=2\left(q_{1} p_{4}-\zeta_{1} \zeta_{2}\right) \mapsto-2 \xi_{4} \partial_{1} ; \\
& \left\{q_{2}, F\right\}_{k . b .}=2\left(q_{2} p_{4}+\zeta_{1} \eta_{3}\right) \mapsto-2 \xi_{4} \partial_{2} ; \\
& \left\{q_{3}, F\right\}_{k . b .}=2\left(q_{3} p_{4}+\zeta_{2} \eta_{3}\right) \mapsto-2 \xi_{4} \partial_{3} ; \\
& \left\{p_{i}, F\right\}_{k . b .}=0 \quad \text { for } i=1,2,3,4 .
\end{aligned}
$$

So we get $K_{f}$, while the remaining brackets lie in $\mathfrak{s v e c t}(0 \mid 3)$.

\section{REFERENCES}

[ALSh] Alekseevsky D., Leites D., Shchepochkina I., Examples of infinite-dimensional simple Lie superalgebras of vector fields. C.R. Acad. Bulg. Sci., v. 33, N 9, 1980, 1187-1190 (in Russian) MR 82k:17010

[BL] Bernstein J., Leites D., Invariant differential operators and irreducible representations of Lie superalgebras of vector fields. Serdika, v.7, 1981 320-334 (in Russian); Sel. Math. Sov., v. 1, N 2, 1981, 143-160 MR 84i:17015

[CK] Cheng, Shun-Jen; Kac, Victor G. A new $N=6$ superconformal algebra. Comm. Math. Phys. 186 (1997), no. 1, 219-231 MR 99f:17029

[GLS] Grozman P., Leites D., Shchepochkina I., Lie superalgebras of string theories. hep-th 9702120

[GPS] Gomis J., París J., Samuel S., Antibracket, antifields and gauge-theory quantization, Phys. Rep. 259 (1995), no. 1-2, 145 pp. MR 96e:81223

[K1] Kac V. G., Lie superalgebras. Adv. Math. v. 26, 1977, 8-96 MR 58:5803

[Ko1] Kotchetkoff Yu. Déformations de superalgèbres de Buttin et quantification. C.R. Acad. Sci. Paris, Sér. I, 299: 14 (1984), 643-645 MR 85k:17021 
[Ko2] Kochetkov Yu. Deformations of Lie superalgebras. VINITI Depositions, Moscow (in Russian) $1985,384-85$

[KL] Kotchetkov Yu., Leites D., Simple Lie algebras in characteristic 2 recovered from superalgebras and on the notion of a simple finite group. In: Kegel O. et.al. (eds.) Proc. Internat. algebraic conference, Novosibirsk, August 1989, Contemporary Math. AMS, 1992, (Part 2), v. 131, 59-67 MR 93g:17035

[L] Leites D., Introduction to the theory of supermanifold. Uspekhi Mat. Nauk, v. 35, 1, 1980, 3-57 MR 81j:58003

[L1] Leites D., New Lie superalgebras and mechanics. Soviet Math. Doklady, v. 18, N5, 1977, $1277-1280$

[L2] Leites D., Lie superalgebras. In: Current Problems of Mathematics. Recent developments, v. 25, VINITI, Moscow, 1984, 3-49 (English translation = JOSMAR, v. 30(6), 1985, 24812512) MR 86f: 17019

[L3] Leites D., Quantization. Supplement 3. In: Berezin F., Shubin M. Schrödinger equation, Kluwer, Dordrecht, 1991, 483-522

[LSe] Leites D., Serganova V., Metasymmetry and Volichenko algebras, Phys. Lett. B, 1990, v. 252(1), 91-96 MR 92b:17005

[LSh1] Leites D., Shchepochkina I., Quivers and Lie superalgebras, Czech. J. Phys. vol 47, n 12, 1997, 1221-1229 MR 99c:16012

[LSh2] Leites D., Shchepochkina I., Deformations of simple vectorial Lie superalgebras (to appear)

[LSh3] Leites D., Shchepochkina I., Automorphisms and real forms of simple vectorial Lie superalgebras (to appear)

[LSh4] Leites D., Shchepochkina I., Classification of simple vectorial Lie superalgebras (to appear)

[M] Manin Yu. I., Gauge fields and complex geometry, 2nd ed, Springer, 1996

[Sh1] Shchepochkina I., Exceptional simple infinite-dimensional Lie superalgebras. C. R. Bulg. Sci., 36, 3, 1983, 313-314 MR 85i:17024

[Sh2] Shchepochkina I., Maximal subalgebras of simple Lie superalgebras. In: Leites D. (ed.) Seminar on Supermanifolds vv.1-34, 1987-1990, v. 32/1988-15, Reports of Stockholm University, 1-43 (hep-th 9702120)

[ShP] Shchepochkina I., Post G., Explicit bracket in an exceptional simple Lie superalgebra, Internat. Journal of Algebra and Computations (to appear); physics 9703022

[St] Sternberg S., Lectures on differential geometry, Chelsea, 2nd edition, 1983 MR 88f:58001

[W] Weisfeiler B., Infinite dimensional filtered Lie algebras and their relation with the graded Lie algebras, Funkcional. Anal. i Prilozhen. 2, n.1, 1968, 94-95 (in Russian) MR 38:1134

On leave of absence from the Independent University of Moscow

Correspondence: c/o D. Leites, Department of Mathematics, University of Stockholm, Roslagsv. 101, Kräftriket hus 6, S-106 91, Stockholm, Sweden

E-mail address: mleites@matematik.su.se

E-mail address: lra@paramonova,mccme.ru 